\title{
BIOMETRICS SECURITY BASED ON FACE RECOGNITION
}

\author{
A THESIS REPORT \\ Submitted by \\ ZIAUL HAQUE CHOUDHURY
}

Under the guidance of

Dr. K.M.MEHATA

In partial fulfillment for the award of the degree of

MASTER OF TECHNOLOGY (BY RESEARCH)

in

INFORMATION TECHNOLOGY

\section{B.S.ABDUR RAHMAN UNIVERSITY}

(B.S. ABDUR RAHMAN INSTITUTE OF SCIENCE \& TECHNOLOGY)

(Estd. U/s 3 of the UGC Act. 1956)

www.bsauniv.ac.in

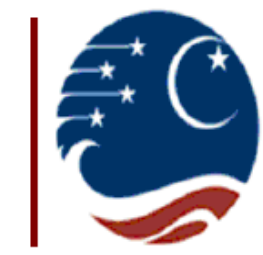

JUNE 2013 


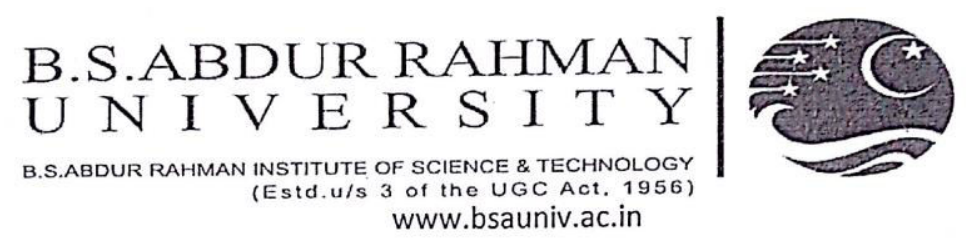

CERTIFICATE

This is to certify that all the corrections and suggestions pointed out by the External Examiners have been incorporated in the thesis titled "BIOMETRICS SECURITY BASED ON FACE RECOGNITION" submitted by Ziaul Haque Choudhury, (RRN:1067201).

Place: Chennai

Date: 23.09.13.

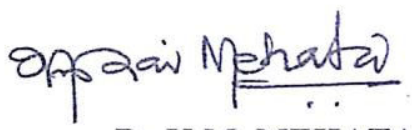

Dr.K.M. MEHATA SUPERVISOR

Dean,School of Computer, Information and Mathematical Sciences, B.S. Abdur Rahman University, Vandalur, Chennai -600 048. 


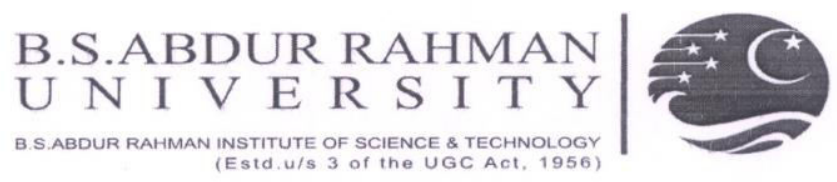

PROCEEDINGS OF THE M.TECH. (BY RESEARCH) VIVA-VOCE EXAMINATION OF Mr. ZIAUL HAQUE CHOUDHURY HELD AT 11.30 A.M. ON 23-09-2013 IN THE DEPARTMENT OF COMPUTER SCIENCE AND ENGINEERING

The M.Tech. (By Research) Viva-Voce Examination of Mr. Ziaul Haque Choudhury (RRN:1067201) on his M.Tech. (By Research) Thesis Entitled " Biometrics Security Based On Face Recognition" was conducted on 23-09-2013 at 11.30 A.M. in the Department of Computer Science And Engineering.

The following Members of the Oral Examination Board were present:
1. Dr.S. Mercy Shalini
Examiner (within state)
2. Dr.S. Bose
Subject Expert
3. Dr.K.M.Mehata
Supervisor \& Convener
4. --Nil--
Joint Supervisor (If any)

The research scholar, Mr. Ziaul Haque Choudhury presented the salient features of his M.Tech. (By Research) work. This was followed by questions from the board members. The questions raised by the Examiners were also put to the scholar. The scholar answered the questions to the full satisfaction of the board members.

The corrections suggested by the examiners have been carried out and incorporated in the Thesis before the Oral examination.

Based on the scholar's research work, his presentation and also the clarifications and answers by the scholar to the questions, the board recommends that Mr. Ziaul Haque Choudhury be awarded M.Tech. (By Research) degree in the Faculty of Information Technology.

1. Dr.S. Mercy Shalini

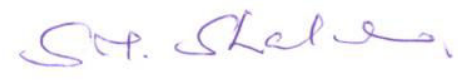

2. Dr.S. Bose

3. Dr.K.M.Mehata

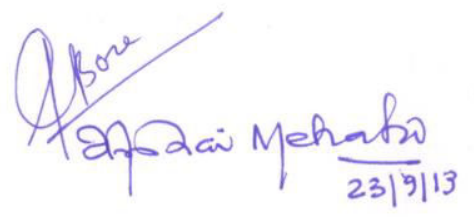




\section{B.S.ABDUR RAHMAN UNIVERSITY}

(B.S. ABDUR RAHMAN INSTITUTE OF SCIENCE \& TECHNOLOGY)

(Estd. u/s 3 of the UGC Act. 1956)

wnw.bsauniv.ac.in

\section{BONAFIDE CERTIFICATE}

Certified that this project report BIOMETRICS SECURITY BASED ON

FACE RECOGNITION is the bonafide work of ZIAUL HAQUE CHOUDHURY

(RRN: 1067201) who carried out the research work under my supervision.

Certified further, that to the best of my knowledge the work reported herein does not form part of any other thesis report or dissertation on the basis of which a degree or award was conferred on an earlier occasion on this or any other candidate.

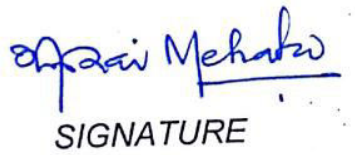

Dr. K.M.MEHATA

SUPERVISOR

Dean SCIS

Department of CSE

B.S. Abdur Rahman University

Vandalur, Chennai - 600048

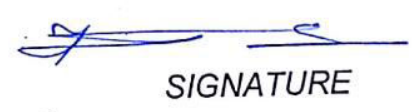

Dr. LATHA TAMILSELVAN HEAD OF THE DEPARTMENT

Professor \& Head Department of IT

B.S. Abdur Rahman University Vandalur, Chennai - 600048 


\section{ACKNOWLEDGEMENT}

This is one of the most significant and delighting moments in my life; making a milestone in one of the longest project, the Master of Technology (By Research) thesis. Finally, I am on the verge of graduating.

I thank my guide Dr. K. M. MEHATA, Dean, School of Computer and Information Sciences, for giving me the research opportunity in Biometrics Security. He has always inspired me with all the interesting and challenging problems. He showed not only which problems we need to solve but how effectively and how efficiently.

I sincerely express my heartfelt gratitude to Prof. JALEES AHMED KHAN TAREEN, Vice Chancellor, B.S. Abdur Rahman University, Chennai.

I sincerely thank Dr. V. M. PERIASAMY, Registrar, for furnishing every essential facility for doing my research project.

I thank Dr. LATHA TAMILSELVAN, Professor and Head, Department of Information Technology, for her eminent support and encouragement throughout my research project.

I express my sincere thanks to the research review committee members, from the Department of Computer Science and Engineering, HOD, Dr. ANGELINA GEETA, Professor, Dr. ANANTHAKUMAR, from the Madras Institute of Technology, Chennai.

I would like to thank all the faculty members and staffs of the Department of Information Technology and Computer Science \& Engineering, B.S.Abdur 
Rahman University for their inspiration, cooperation and provided me all official and laboratory facilities in various ways for the completion of this thesis.

My obligated respect and thanks to my loving parents and sisters for their love, sacrifice, inspiration, suggestions and support. They are my first teachers after I came to this world and have set great examples for me about how to live, study and work.

(ZIAUL HAQUE CHOUDHURY) 


\section{ABSTRACT}

Biometrics is a rapidly developing technology, which has been broadly applied in forensics such as criminal identification, secured access, and prison security. The biometric technology is basically a pattern recognition system that acknowledges a person by finding out the legitimacy of a specific behavioral or physiological characteristic owned by that person. In this era, face is one of the commonly acceptable biometrics system which is used by humans in their visual interaction and authentication purpose. The challenges in the face recognition system arise from different issues concerned with cosmetic applied faces and of low quality images.

In this thesis, we propose two novel techniques for extraction of facial features and recognition of faces when thick cosmetic is applied and of low quality images. In the face recognition technology, the facial marks identification method is one of the unique facial identification tasks using soft biometrics. Also facial marks information can enhance the face matching score to improve the face recognition performance. When faces are applied by thick cosmetics, some of the facial marks are invisible or hidden from their faces. In the literature, to detect the facial marks AAM (Active Appearance Model) and LoG (Laplacian of Gaussian) techniques are used. However, to the best of our knowledge, the methods related to the detection of facial marks are poor in performance especially when thick cosmetic is applied to the faces. A robust method is proposed to detect the facial marks such as tattoos, scars, freckles and moles etc. Initially the active appearance model (AAM) is applied for facial feature detection purpose. In addition to this prior model the Canny edge detector method is also applied to detect the facial mark edges. Finally SURF is used to detect the hidden facial marks which are covered by cosmetic items. It has been shown that the choice of this method gives high accuracy in facial marks detection of the cosmetic applied faces. 
Besides, another aspect of the face recognition based on low quality images is also studied. Face recognition indeed plays a major rule in the biometrics security environment. To provide secure authentication, a robust methodology for recognizing and authentication of the human face is required. However, there are numbers of difficulties in recognizing the human face and authentication of the person perfectly. The difficulty includes low quality of images due to sparse dark or light disturbances. To overcome such kind of problems, powerful algorithms are required to filter the images and detect the face and facial marks. This technique comprises extensively of detecting the different facial marks from that of low quality images which have salt and pepper noise in them. Initially (AMF) Adaptive Median Filter is applied to filter the images. The filtered images are then extracted to detect the primary facial feature using a powerful algorithm like Active Shape Model (ASM) into Active Appearance Model (AAM). Finally, the features are extracted using feature extractor algorithm Gradient Location Orientation Histogram (GLOH).

Experimental results based on the CVL database and CMU PIE database with 1000 images of 1000 subjects and 2000 images of 2000 subjects show that the use of soft biometrics is able to improve face recognition performance. The results also showed that 93 percentage of accuracy is achieved. Second experiment is conducted with an Indian face database with 1000 images and results showed that 95 percentage of accuracy is achieved. 


\section{TABLE OF CONTENTS}

CHAPTER NO
TITLE

ABSTRACT

LIST OF FIGURES

LIST OF TABLES

LIST OF ABBREVIATION
PAGE NO.

vii

xiii

xvi

xvii

INTRODUCTION

Networks Security 1

Networks Security Concepts 1

Security Issues and challenges $\quad 2$

1.3.1 Information Security 2

1.3.2 Computer Security 2

1.3.3 Internet Security 4

BIOMETRICS SECURITY 5

$2.1 \quad$ History of Biometrics

$\begin{array}{lll}2.2 & \text { Biometrics Security } & 6\end{array}$

$\begin{array}{lll}2.3 & \text { Biometrics System } & 7\end{array}$

2.4 A brief review of the major biometrics 8 technologies 
$2.5 \quad$ Differences between behavioral \& physical biometrics

$2.6 \quad$ The major biometric concept

2.7 Applications of biometrics

$2.8 \quad$ Criminal justice system

2.8.1 Finger printing

2.8.2 Face recognition

2.9 Challenges on biometrics security: face recognition

2.9.1 Face recognition

2.9.2 Face recognition in $2 \mathrm{D}$

2.9.3 Face representation features 
4.2.2 Mean shape mapping

4.2.6 Facial marks based matching $\quad 60$

$\begin{array}{lll}4.3 & \text { Experimental results } & 61\end{array}$

$\begin{array}{lll}4.4 & \text { Summary } & 63\end{array}$ QUALITY IMAGES 
$\begin{array}{lll}\text { 5.4.1 Noise removal } & 70\end{array}$

5.4.2 Facial feature detection 73

5.4.3 Designing mean shape \& mask construction $\quad 73$

5.4.4 Gradient location and orientation histogram $\quad 75$

5.4.5 Blob classification for facial marks $\quad 78$

5.4.6 Face based matching for facial marks $\quad 79$

$\begin{array}{lll}5.5 & \text { Experimental results } & 81\end{array}$

$\begin{array}{lll}5.6 & \text { Summary } & 83\end{array}$

$6 \quad$ CONCLUSIONS AND FUTURE DIRECTION

84

$\begin{array}{lll}6.1 & \text { Conclusion } & 84\end{array}$

$\begin{array}{lll}6.2 & \text { Future direction } & 85\end{array}$

BIBILOGRAPHY $\quad 86$

LIST OF PUBLICATIONS $\quad 99$

$\begin{array}{ll}\text { TECHNICAL BIOGRAPHY } & 100\end{array}$ 


\section{LIST OF FIGURES}

FIGURE NO.

2.1 statics of biometrics users in worldwide in the year 2012

2.2 Examples of different types of biometric authentication

2.3 Example applications using face biometric

2.4 Face recognition performance in FRVT 2002

4.1 Examples of facial marks Scar, mole, and freckles etc.

4.2 Examples of textual query and a schematic of face region segmentation

4.3 Three different types of example of facial 49 marks results. Full face, partial face, and off-frontal face

4.4 Schematic of automatic facial mark extraction process

4.5 Schematic of texture mapping process 52 using the triangular Barycentric coordinate system

4.6 Schematic of the mean face construction 
4.7 Mark classification using morphology $\quad 57$

4.8 Examples of mark detection and 58 classification results

4.9 Mark detection graph 58

4.9.1 Schematic of the mark based indexing 60 scheme

4.9.2 Examples of face image retrieval result. 63

5.1 Example of low quality image with 64 different facial marks

5.2 Example of textual query and a schematic $\quad 66$ of face region segmentation

5.3 Over all structure of our proposed method 68

5.4 Shows salt and pepper noise images 68

5.5 Different facial marks on the mean face 69 image

5.6 Schematic of Automatic Facial Mark 70 Extraction Process.

$\begin{array}{lll}5.7 & \text { Salt and pepper noise filtered with AMF } & 71\end{array}$

$\begin{array}{lll}5.8 & \text { shows histogram for noisy color image } & 72\end{array}$

5.9 shows histogram for noiseless color 72

5.9.1 Schematic of texture mapping process 74 using the triangular Barycentric 
coordinate system

5.9.2 Schematic of the mean face construction

5.9.3 Marks detection performance graph

5.9.4 Mark classification using morphology.

5.9.5 Examples of facial marks which are detected from face image

5.9.6 Schematic of the mark based indexing scheme

5.9.7 Face matching and retrieval results 


\section{LIST OF TABLES}

4.9.2 Proposed facial marks matcher \& face

62

recognition accuracy

5.9.3.1 Mark Detection Accuracy (MDA)

76

in percentage 


\section{LIST OF ABBREVIATION}

$\begin{array}{lll}\text { AAM } & - & \text { Active Appearance Model } \\ \text { PCA } & - & \text { Principle Component Analysis } \\ \text { SURF } & - & \text { Speed Up Robust Feature } \\ \text { CCD } & - & \text { Charge Coupled Device } \\ \text { DB } & - & \text { Data Base } \\ \text { ASM } & - & \text { Active Shape Model } \\ \text { SIFT } & - & \text { Scale Invariant Feature Transform } \\ \text { GLOH } & - & \text { Gradient Location Orientation Histogram } \\ \text { 3D } & - & 3 \text { Dimensional } \\ \text { AMF } & - & \text { Test Environment as a Service } \\ \text { SMT } & - & \text { Scars, Marks, and Tattoos }\end{array}$




\section{INTRODUCTION}

\subsection{NETWORK SECURITY}

Network security lies in the provisions \& policies took over by a network administrator to protect and keep tabs on unauthorized access such as modification, misuse, or denial of a computer network and network accessible resources. Network security necessitates the authorization of access to data in a network, which is controlled by the network administrator. Users pick out or are allotted an ID and password or other authenticating information that permits them access to information and programs. Network security addressing an assortment of computer networks, both private and public that are applied in everyday tasks carrying on communications and transactions among individuals, businesses, and government agencies. Networks can be private, such as inside a company, and others which might be exposed to public access. Network security is necessitated in enterprises, organizations, and other parts of the institutions. It does as its title explicates, it secures the network, as well as defend and oversee the operations being performed. The most simple and common way of defending a network resource is by allotting it a corresponding password and unique name.

\subsection{Network security concepts}

Network security commences with authenticating the user, through the 'username' and a 'password', which is referred as one factor authentication. With two factor authentication, something the user 'has' is also applied (e.g. 'dongle', a security token, an ATM card, or a mobile phone); and with three factor authentication, another criterion is also applied (e.g. retinal scan, face recognition or a fingerprint, etc.). Once authenticated, a firewall imposes access policies such as what services are permitted to be accessed by the network users. 


\subsection{SECURITY ISSUES \& CHALLENGES IN NETWORKS \& ENTERPRISES}

\subsubsection{Information Security}

"Information security is the practice of defending information from unauthorized access, use, modification, disclosure, perusal, inspection, Denial of use of knowledge, misuse, Facts, recording or destruction." From the above definition, Information Security does guarantees protection. The confidentiality, integrity and availability (CIA) triad is one of the core principles of information security. In the year 1992 and subsequently revised in 2002, the OECD's guidelines for the Security of Information Systems and Networks proposed the nine generally accepted principles: a) Awareness, b) Responsibility, c) Response, d) Ethics, e) Democracy, f) Security Management, g) Risk Assessment, h) Reassessment, and i) Security Design and Implementation. Building upon those, in the year 2004 the NIST's Engineering Principles for Information Technology Security (EPITS) proposed 33 principles. In the year 2002, Donn Parker suggested an alternative model for the classic CIA triad that he named the six atomic elements of information. The elements are i) confidentiality, ii) possession, iii) integrity, iv) authenticity, v) availability, and vi) utility. The merits of the Parkerian hexad are subjects of debate amongst security professionals.

\subsubsection{Computer security}

With the introduction of the computer, the requirement for automated tools for defending files and other information stored on the computer became apparent. This is particularly the case for a shared system, and the requirement is even more discriminating for systems that can be got at over a public telephone network, the Internet, or data network. The aggregation of tools planned to protect data and to thwart hackers is what constitutes the computer security. 


\subsubsection{Security by design}

Some of the techniques that are commonly used to implement computer security include:

$\rightarrow$ The principle of least perquisite, where each part of the system has only the perquisite that are required for its function. That way even if a assailer benefits access to that part, they have only bounded access to the whole system.

$\rightarrow$ Automated theorem proving to demonstrate the correctness of crucial software subsystems.

$\rightarrow$ Unit testing and code reviews to make modules more secure where formal rightness proofs are not possible.

$\rightarrow$ Defense in depth, where the design is such that more than one sub-system requires to be breached to compromise the unity of the system and the information it holds.

$\rightarrow$ Default secures settings and design to 'fail secure' rather than 'fail insecure'. Ideally, a secure system should need a deliberate, knowledgeable, conscious, and free decision on the part of legitimate authorities in order that make it insecure.

$\rightarrow$ Audit trails tracking system activity, so that when a security breach goes on, the mechanism and extent of the breach can be found out. Putting in audit trails remotely, where they can only be appended, it can keep intruders from covering their tracks.

$\rightarrow$ Full revelation to ensure that when bugs are found the 'window of vulnerability' is kept as short as potential. 


\subsubsection{Internet security}

Security is influenced by the introduction of spread out systems and the use of networks and communications for carrying data between the end user and the computer and between the computer and computer. Network security evaluation is needed to protect data during their transmission. In fact, the term network security is somewhat misleading because virtually all government, business, and academic organizations interlink their data processing equipment with a collection of interlinked networks. Such a collection is frequently referred to as an internet and the term internet security is applied. 


\section{BIOMETRICS SECURITY}

\subsection{HISTORY OF BIOMETRICS}

The term "Biometrics" is derived from the Greek words "bio" means "life" and "metrics" means "to measure". Automatized biometric systems have become obtainable only over the last few decades due to important advances in the field of computer science. Lots of these new automated techniques, nevertheless, are based on thoughts that were originally imagined hundreds or even thousands of years ago. One of the most former and most basic examples of a characteristic that is utilized for recognition is the human face. Since the commencing of civilization, humans have applied faces to identify known 'familiar' and unknown 'unfamiliar' individuals. Figure 2.1, the statics of biometrics users in worldwide in the year of 2012.

\section{One Billion People in Developing Countries Have Taken Part in Biometric Identification} Programs: The Use of Biometrics Technology for Development, LMICS, 2012.

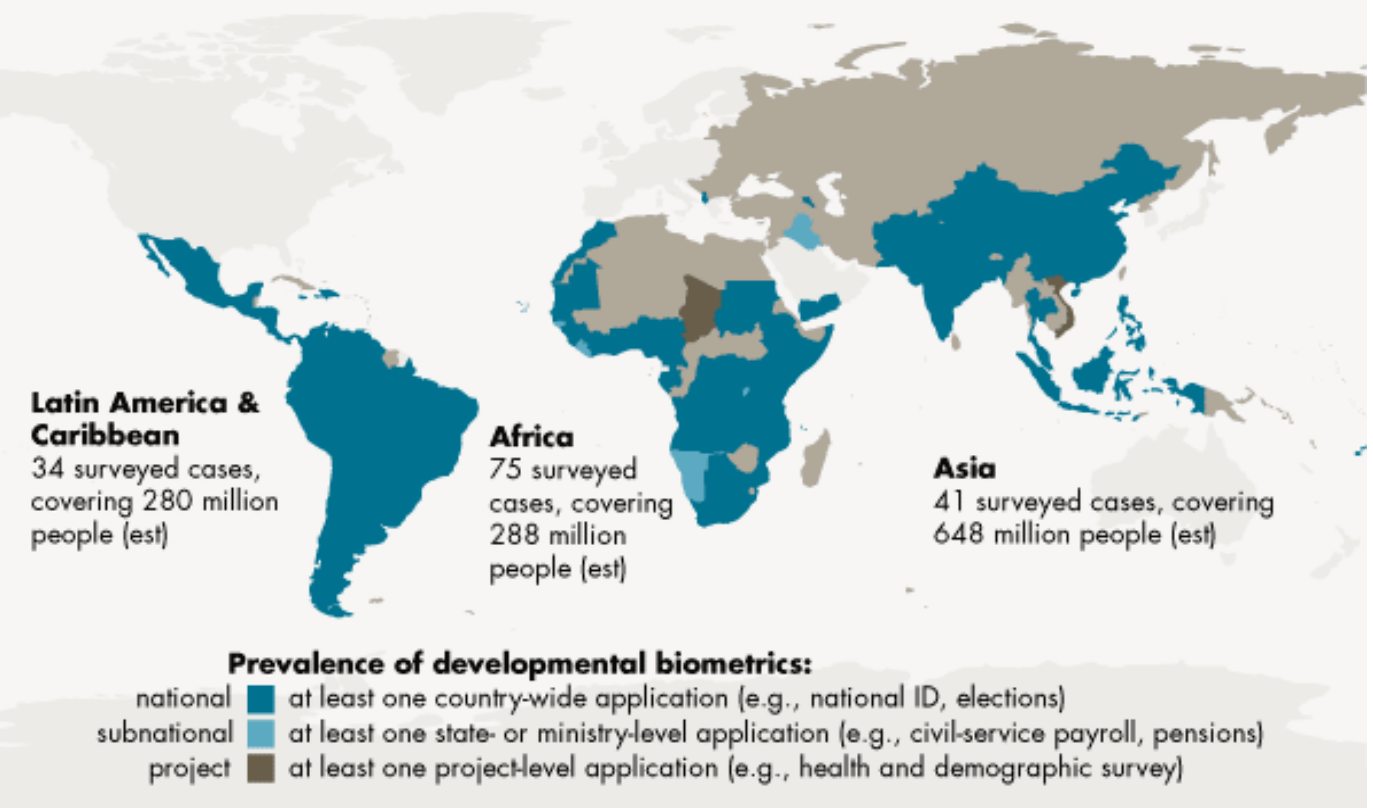

Figure 2.1, statics of biometrics users in worldwide in the year 2012 
This simple task became more and more challenging as the human population increased and as more commodious methods of travel brought in many new individuals into small once communities. The conception of human to human acknowledgement is also seen in behavioral very common biometrics such as gait and speaker recognition. Individual use these features, somewhat without awareness to recognize known individuals on a day by day basis.

\subsection{BIOMETRICS SECURITY}

A biometric is a mensuration of biological features such as fingerprint, iris pattern, retina image and face or hand geometry or behavioral features such as gait, voice, or signature. Biometric technology utilized these characteristics to recognize individuals automatically. Most preferably the characteristic should be universally present and unique to the individual, easily measurable and stable over time. No biometric features have been officially demonstrated to be unique, even though they are usually sufficiently distinct for practical applies. Dissimilar biometrics will be more desirable for dissimilar applications depending. For example, on whether the objective is to discover someone with their cooperation or from a distance without their cognition. As illustrated below, biometrics can be applied to answer two principal questions:-

$\rightarrow$ Are you who you claim to be?

$\rightarrow$ Who are you?

\section{$\rightarrow$ Are you who you claim to be?}

Confirming that someone is who they claim to be normally relied on security pass, or something that they know, that is password. Absolute confidence cannot be provided by either of them. For instance, security passes can be skipped and passwords are sometimes unknowingly written down. Biometric technology offers an extra level of assurance, even if the person's 
characteristics or a password are easily known and can therefore be replicated. To affirm an individual's identity, their biometrics is scanned and converted into electronic form and kept either on a card that remains in their possession or in a database. On requesting access to an IT system or a building, the biometric is scanned again and equated with the record to confirm their individuality. Where the objective is simply to recognize an individual as someone with permission to use the system, there is no actual need to link the biometric data with any other personal information.

\section{$\rightarrow$ Who are you?}

If a database of known individuals has been developed it is possible to answer the question 'who are you?' Against the database in a 'one-to-many' search, the biometric of the unknown person is compared. Their identity can be determined if their biometric has been entered into the database on a previous occasion; this is much quicker than a manual system. High quality data are needed if the database searches are to give accurate results.

\subsection{BIOMETIRC SYSTEM}

A biometric recognition or simply a biometric system is a pattern recognition system that authenticates an individual based on his biometric traits. The biometric recognition technology consists of four main modules: (a) sensor that captures samples of a biometric trait, (b) feature extraction module that extracts certain salient features of the biometric sample captured by the sensor, (c) system database that stores the features extracted by the feature extraction module, and (d) matcher module that equalizes the features extracted from the biometric samples with the features stored in the system database. See Figure 2.2 , for an illustration of a typical biometric recognition technology. 


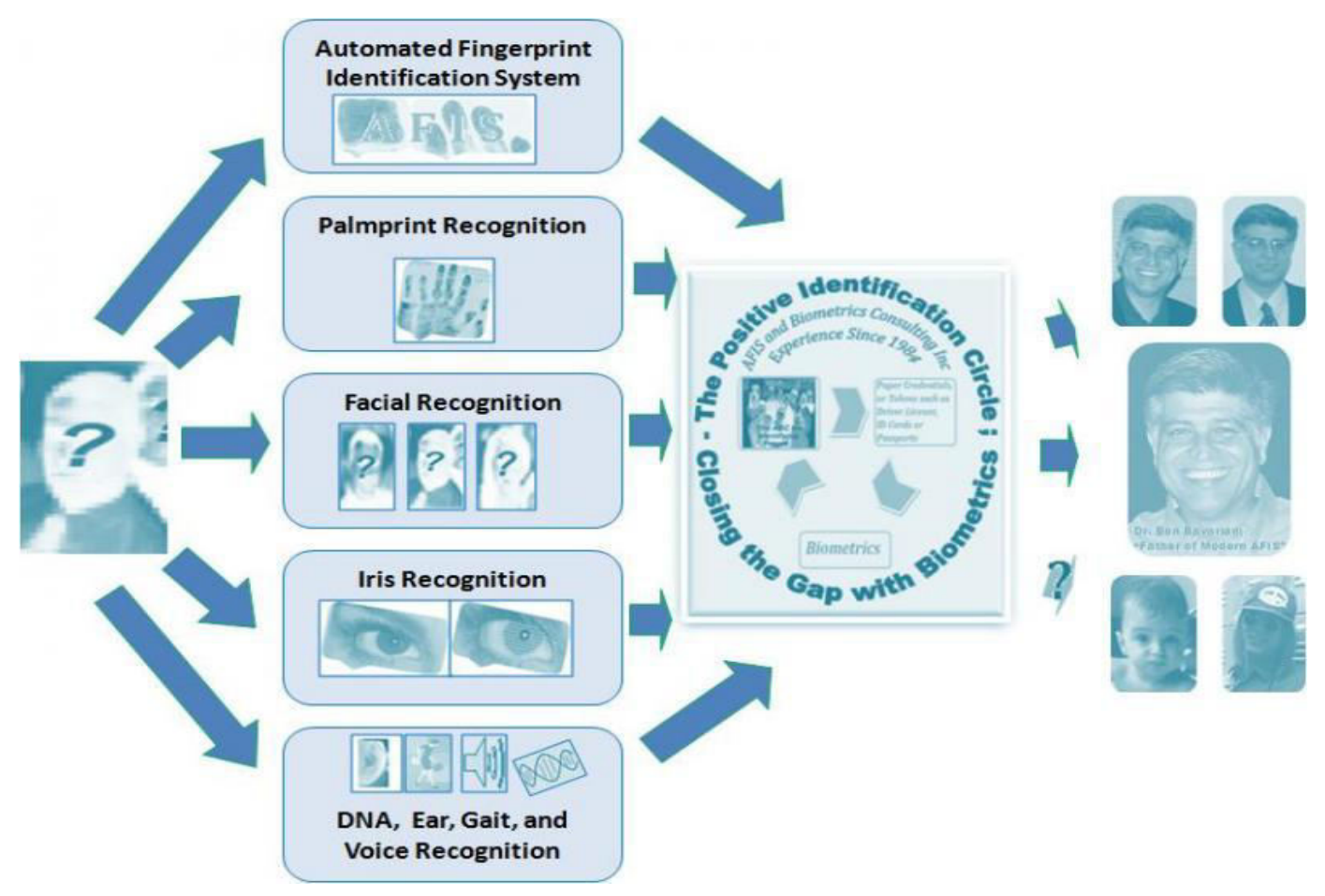

Figure 2.2, Examples of different types of biometric authentication

\subsection{A BRIEF REVIEW OF THE MAJOR BIOMETRIC TECHNOLOGIES}

There are a total of seven major biometric technologies available today. They are:

$\rightarrow$ Fingerprint recognition;

$\rightarrow$ Hand geometry recognition;

$\rightarrow$ Facial recognition;

$\rightarrow$ Iris and retina recognition;

$\rightarrow$ Voice recognition;

$\rightarrow$ Keystroke recognition;

$\rightarrow$ Signature recognition; 
Of these technologies, hand geometry recognition, fingerprint recognition and iris recognition are most predominant. For DNA recognition (examining the unique strands found in DNA samples), earlobe recognition (examining the geometry of the earlobe) and gait recognition (the way and manner in which somebody walks), considerable time and effort is being invested in biometric technologies of the future. About the biometric technology a brief description is given below.

\section{Fingerprint recognition}

The location and determination of the unique characteristics of the fingerprint are involved in fingerprint recognition. Various 'valleys' and 'ridges', which form the basis for the loops, arches and swirls on your fingertip is included in the fingerprint. Different kinds of breaks and discontinuities are contained in the ridges and valleys. These are called as 'minutiae'. The unique features are located and determined from these minutiae. Two types of minutiae are there: bifurcations (the location where a single ridge splits into two ridges), ridge endings (the location where the ridge actually ends).

\section{Hand geometry recognition}

Hand geometry identification requires looking for unique features in the structure of the hand. These features contain the length, thickness and width of the finger, the distances between the hand's overall bone structure and finger joints. Of these unique characteristics, a 3-dimensional image is taken. As other identifiers, the hand does not contain as many unique characteristics. 


\section{Facial recognition}

Facial recognition contains taking many images or pictures of the face and extracting the unique facial features as well as distances from or between the nose, ears, mouth, eyes and cheeks.

\section{Iris and retinal recognition}

Examining the unique features of the iris is entailed in iris recognition. The colored section between the white region of the eye (also known as the sclera) and the pupil is the iris. To control the size of the pupil (the part of the eye that allows light to pass through) is its primary purpose. The trabecular mesh-work (the tissue that gives the iris its 'radial' impression) is included in the unique features of the iris and as well as other physiological properties that is freckles, furrows, rings, and the corona. Examining the pattern of blood vessels in the retina that is located at the back of the eye is involved in retinal recognition. At the juncture of the optic nerve (the area where the nerve leaves the brain and enters the eye) is focused on the examination.

\section{Voice recognition}

With voice recognition, it is the unique patterns of an individual's voice, as produced by the vocal tract, which is examined. A text phrase is usually recited in order to capture the voice inflections. The vocal tract consists of the oral pharynx, nasal pharynx, laryngeal pharynx, oral cavity, and the nasal cavity.

\section{Keystroke recognition}

Keystroke identification works by analyzing the unique way in which an individual character on a computer keyboard. Variables contain typing speed, 
the distance of time that keys are held down, and the time taken between c successive keystrokes.

\section{Signature recognition}

Signature recognition analyzes the way and manner in which we sign our name. Unique features include alteration in timing, speed and pressure during the signing process. It is significant to understand that it is not the signature itself that is examined.

\subsection{DIFFERENCES BETWEEN BEHAVIORAL AND PHYSICAL BIOMETIRCS}

The given biometric technologies are divided in two categories: behavioral biometrics and physical biometrics. In general, As captured by a biometric system, generally behavioral biometrics can be defined as the non-biological or non- physiological features (or unique identifiers). This category includes signature as well as keystroke recognition as behavioral biometrics also covers any mannerisms or behavior displayed by an individual. As captured by a biometric system, physical biometrics may be defined as the biological and physiological features (or unique identifiers). Hand geometry recognition, fingerprint recognition, facial recognition, iris and retinal recognition, and voice recognition are included in this category.

\subsection{THE MAJOR BIOMETIRC CONCEPTS}

It is important that one has a basic understanding of the key concepts that are associated with biometrics, when examining the various biometric technologies and systems. Each of these concepts is discussed below: 
$\rightarrow$ Verification and identification

$\rightarrow$ Biometric templates

$\rightarrow$ The processes of enrolment, verification and authorization;

$\rightarrow$ Biometric performance standards;

\section{Verification and identification}

The aim of the verification process is to establish someone's claimed identity. It assigns you a number when you first enroll in a biometric system, which is linked to your biometric template. On the basis of this number the database containing your template is searched. You will be extended a given service or privilege, if a positive match is established. A one-to-one (or 1:1) relationship is said to exist as the number is linked to the template. The identification process looks to establish someone's identity as its name suggests. The entire database needs to be searched in order for the system to 'recognize' you in the absence of a unique number. A one-to-many $(1: n)$ relationship is said to exist as the template can belong to anyone in the database. To identify and track known criminals, a good example of a 1: $n$ database is AFIS (Automated Fingerprint Identification Services), which is used by many law enforcement agencies.

\section{Biometric templates}

It takes numerous images/recordings of your biological and non-biological data (i.e. a voice recording or keystroke pattern) when you first enroll in a biometric system. These raw images and recordings are subsequently consolidated into one main image, called as the 'biometric sample'. The unique features (discussed above) are captured and extracted from this sample. Next, for the purposes of verification and identification, they are converted to a 'biometric template'. The biometric system does not contain actual images of the biological or non biological data, it only stores the mathematical files. Depending on the 
biometric system in use, the type of mathematical file is created in which binary mathematical files are created by fingerprint recognition and hand geometry recognition systems. Hexadecimal files are generated by iris recognition systems.

\section{The processes of enrollment, verification and authorization}

When you try to enter a high security area, your finger print need to be registered and added to a database. This process is known as enrollment. Various images of the fingerprint are taken, that are combined in a single biometric sample. To extract the unique features from the given sample, a mathematical formula or extraction algorithm is used next. These features are subsequently stored in a mathematical file known as an enrollment template that is used for the verification purposes. The next step includes verification. You are required to identify yourself by placing your finger on a scanner of fingerprint in order to enter a high-security area. 'An image' of your print will be captured by the scanner's sensor. The unique features are extracted from the image subsequently by the extraction algorithm and store these in a file known as the verification template. In order to determine the extent to which they match, the next stage includes comparing the verification template to the enrollment template, which are obtained with the help of a matching algorithm. Based on the amount of overlap between both templates, the latter gives a score. If an agreed value (or threshold) is lesser than this score, you are authorized to enter the area. If the threshold value is higher than the score, you are denied access (and the verification process may be repeated). Although much happens during the processes of enrollment, verification and authorization, only a few seconds is taken to complete. It is important to remember that an enrollment template is never completely $(100 \%)$ similar to the verification template at this point. 


\section{Biometric performance standards}

Based on the performance of several standards, biometric systems are rated. The most important are the False Acceptance Rate (FAR), the False Rejection Rate (FRR) and the Equal Error Rate (EER) as shown in figure 1. It can be defined as the probability of a registered user, as it is wrongly rejected by the biometric system, the FRR, which are also known as a type 1 error. As the probability of an impostor being wrongly authorized by the biometric system, the FAR (also known as type 2 errors) can be defined. The probability of the FAR and the FRR is reflected by the EER (or crossover rate) which is almost the same. There are also other standards of biometric. To Enroll rate (FTE), for example, the failure, which defines the statistical probability that a person is simply unable to enroll in a biometric system. For the system to capture, this may be attributable to the fact that the person in question does not have enough unique features. The Ability to Verify rate (ATV) indicates the overall percentage of users that can be verified by a system of biometric. The ATV can also be the combination of the FTE and the FRR. Mathematically, this relationship can be represented as follows:

$$
A T V=\left[(1-F T E){ }^{*}(1-F R R)\right]
$$

\subsection{APPLICATIONS OF BIOMETRICS}

For use in access/l.D. cards and for small-scale security of buildings and IT systems, there is growing interest in the use of biometrics. At a national level, on current and potential large-scale applications this brief is focused. These contain the immigration and asylum, Criminal Justice System and port and border security. The law has regulated the collection of fingerprints by the police and immigration service. General legislation regulates other biometric technology. 


\subsection{CRIMINAL JUSTICE SYSTEM}

\subsubsection{Fingerprinting}

Automated fingerprinting is the only biometric in general use (see box on NAFIS opposite) at a national level in the UK. Prisons already take ink fingerprints from prisoners who are convicted. As proof, these can be compared against the police database in which the intended person is being held. A system which is automatic would give rapid confirmation of a person's identity and allow information about individuals to be shared quickly and easily.

\subsubsection{Face Recognition}

Everyone is charged or convicted of an offence by the police through photographs. Digital databases of static photos are already held by some police forces. To set up a national face recognition database might be an option, comparable with NAFIS in that it could carry out searches based on static images taken in police stations. Face recognition technology could also be used to complement the estimated 1 million CCTV cameras already in operation in the UK, by recognizing individuals covertly at a distance. This is a greater technical challenge than the use of static images collected with the knowledge and co-operation of the individual. Potentially, covert recognition could be used in real time, allowing the instant identification of individuals, or offline, enabling hours of CCTV recordings to be searched quickly and without human fatigue.

\subsection{CHALLENGES ON BIOMETIRC SECURITY: FACE RECOGNITION}

To establish a subject's identity based on facial characteristics is the ability of face recognition. Various techniques from different research fields are required in automated face recognition such as (i) computer vision, (ii) machine learning, 
(iii) pattern recognition, and (iv) image processing. From a number of subjects, face images are enrolled into the system as gallery data in a typical face recognition system, and using a one-to-one or one-to- many scheme, the test subject's face image (probe image) is matched to the gallery data. The one-tomany and one-to-one matching are called identification and verification respectively. To interact with each other, face recognition is one of the fundamental methods used by human beings. Using a pair of photographs dates back to 1871 in a British court [28], attempts are made to match faces. For the purpose of automatic person recognition, with still and video images, techniques for automatic face recognition have been developed over the past three decades. A wide range of applications, including enforcement of law, applications of civil, and systems of surveillance are there in face recognition. To smart home systems face recognition applications have also been extended. For better interactive communications between human and machines [29], the recognition of the human face and expression is used. Using the face Figure 2.3 shows some applications of biometrics.

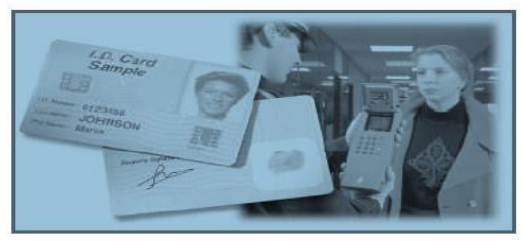

(a)

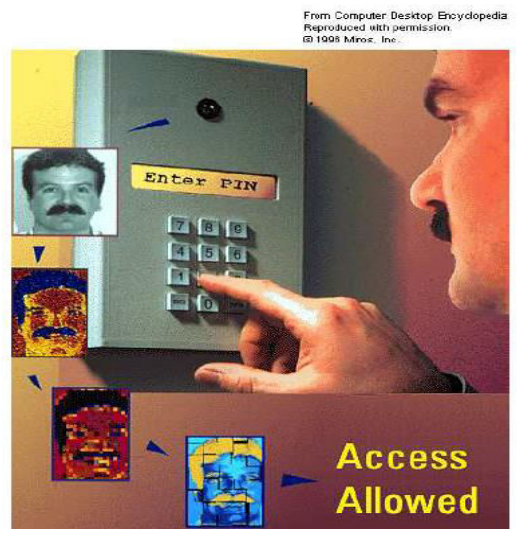

(c)

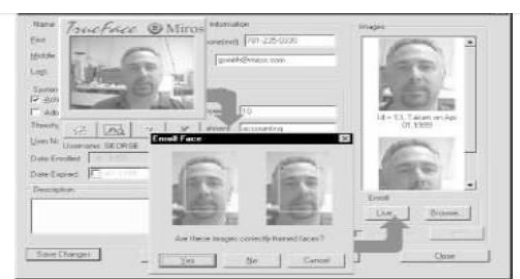

(b)

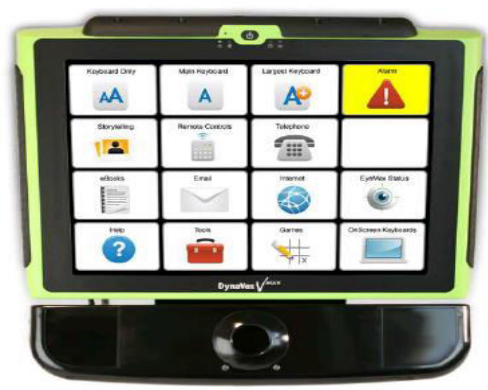

(d) 
Figure 2.3, Example applications using face biometric: (a) ID cards, (b) face matching and retrieval (c) access control and (d) Dyna Vox Eye Max system (controlled by eye gaze and blinking.)

The face has several advantages that make it one of the most preferred biometric traits. The face biometric is easy to capture even at a very far distance. The internal feelings (emotion) of the subject (e.g., happiness or sadness) and the person's age are also conveyed besides the identity of the face. This makes an important topic in human computer interaction as well as person recognition of face recognition. There has been a significant improvement in performance of face recognition for the past one decade for many applications [29] [30] but it is still below acceptable levels. To overcome the performance bottleneck in 2D still face recognition, video input, and different features (e.g., skin texture), recent efforts have focused on using 3D models. This chapter starts with a survey of recognition of face in 2D and presents the challenges in problems of face recognition.

\subsubsection{Face Recognition}

Using a one-to-one or one-to-many scheme, images of faces from a number of subjects are enrolled into the system as gallery data and the face image of a test subject (probe image) is matched to gallery data in a typical scenario of face recognition. Three distinct modalities are there that are used in recognition of face applications: 2D, 3D, and video. The face recognition problems will be reviewed in these domains in the following sections.

\subsubsection{Face Recognition in 2D}

Using 2D still images, face recognition has been well studied for over a decade [42] [43] [44]. Face recognition systems based on 2D still image, a snapshot of a 
user is acquired and compared with a gallery of snapshots to establish an identity of a person. The user is expected to be cooperative and provide a frontal face under uniform lighting conditions with a simple background to enable the capture and segmentation of a high quality image of the face in this procedure. Although, it is now well known that the performance of the single-shot 2D image based face recognition systems [29] can be drastically degraded by small variations in pose and lighting. $2 \mathrm{D}$ face recognition is usually categorized according to the number of images used in matching Some of the well-known algorithms for $2 \mathrm{D}$ face recognition are based on Principle Component Analysis (PCA) [42] [43], Linear Discriminant Analysis (LDA) [45], Elastic Graph Bunch Model (EGBM) [46], and correlation based matching [47]. Continuously 2D face recognition technology is evolving. Identification accuracy of around $70 \%$ was achieved given near frontal pose and normal lighting conditions on a large database (121,589 images from 37,437 subjects, Figure 2.5$)$ in the Face Recognition Vendor Test (FRVT) 2002 [48]. The FRVT 2006 evaluations were performed in a verification scenario and the best performing system showed a 0.01 False Reject Rate (FRR) at a False Accept Rate (FAR) of 0.001 given the high resolution (400 pixels between eyes1) 2D images (by Neven Vision [49]) or 3D images (by Viisage2 [50]). In closed set or open set scenarios, face recognition can be performed. The open set recognition considers the possibility that the probe subject is not enrolled in the gallery but the closed set recognition tests always have the probe subject enrolled in the gallery data. Therefore in retrieving candidate matches in open set recognition tests [51], a threshold value (on match score) is typically used. 


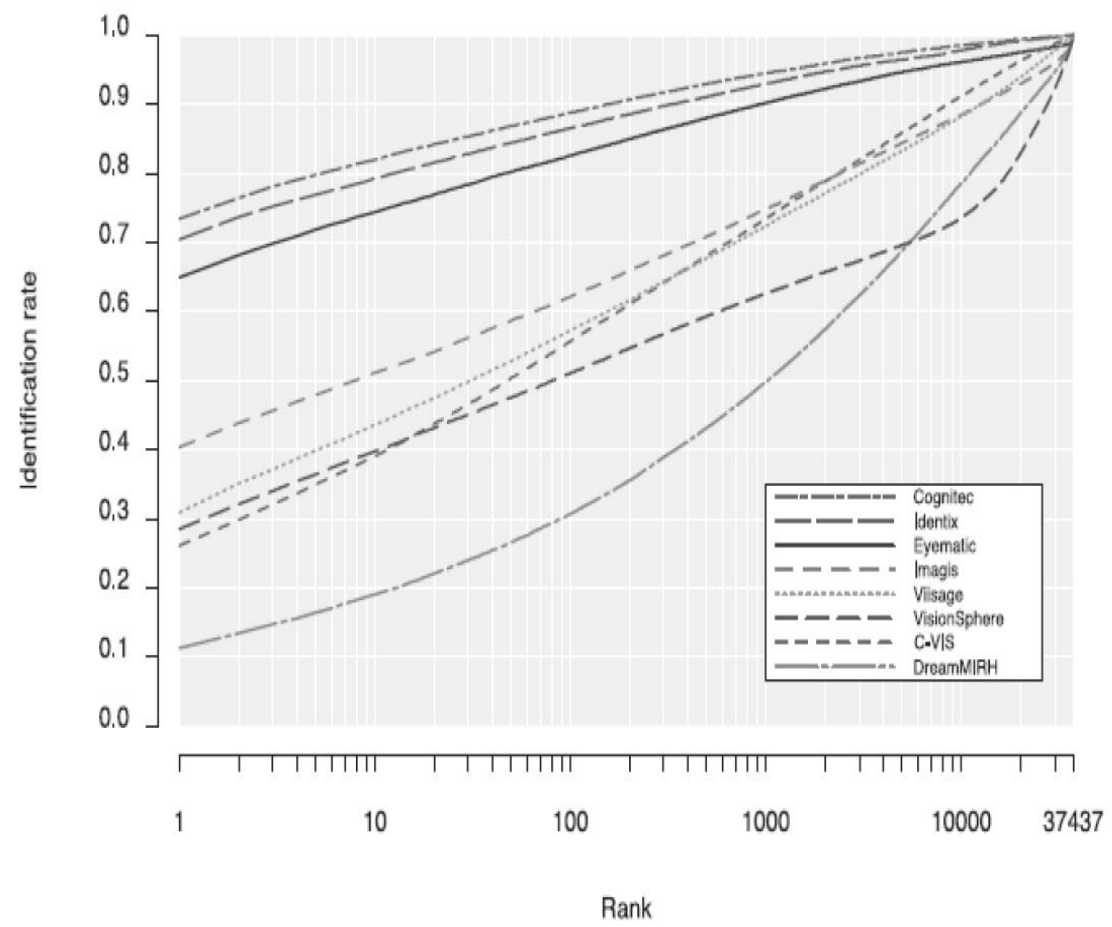

Figure 2.4, Face recognition performance in FRVT 2002 (Snapshot of computer screen) [90].

\subsubsection{Face Representation}

Most face recognition techniques use one of two representation approaches: i) local feature based [52] [53] [54] [55] [56] [57] or ii) holistic based [43] [42] [58] [59]. Local feature based approach to identify local features (e.g., eyes, nose, mouth, or skin irregularities) in a face and generate a representation based on their configuration of geometry. The approach based on holistic localize the face and use the entire face region in the sensed image to generate a representation. For this purpose, a dimensionality reduction technique (e.g., PCA) is used. The information of discrimination present in micro facial features (for instance, speckles or moles) is usually ignored and considered as noise. Applying further transformations on the holistic representation is also a common technique (e.g., LDA). A combination of local and holistic representations has also been studied 
[60] [61]. Local feature based approaches can be further categorized as: i) component based [62] [63], ii) modular [64] [67] [66] [68] [65], and iii) skin detail based [69] [70]. Component based approaches try to identify local facial primitives (e.g., eyes, nose, and mouth) and either use all or a subset of them to generate features for matching. Modular approaches subdivide the face region, irrespective of the facial primitives, to generate a representation. Skin detail based approaches have recently gained attention due to the availability of high resolution (more than 400 pixels between the eyes) face images. Facial irregularities (e.g., freckles, moles, scars) can be explicitly or implicitly captured and used for matching in high/low resolution face images. 


\section{LITERATURE OVERVIEW}

\subsection{Face authentication using different facial features}

To authenticate a person's face in the literature, the authors J. E. Lee, A. K. Jain, and R. Jin et al. (2008) used Scars, marks and tattoos (SMT) are being increasingly used for suspect and victim identification in forensics and agencies of law enforcement. In particular, tattoos are getting serious attention because of their visual and demographic characteristics as well as their increasing prevalence. Scars, marks, and tattoos (SMT) are imprints of skin that have been shown to be useful by law enforcement agencies for identification of a nonskeletalized body of a victim or a suspect using a false identity. The author illustrated the method of detecting SMT provide more discriminative information than the traditional demographic indicators such as age, height, gender, race, and gender to identify a person. To detect the SMT they used Scale Invariant Feature Transform (SIFT) extracts repeatable characteristic feature points from an image and generates descriptors representing the texture around the feature points. They contributed their method based on a CBIR system for tattoo images, with the growing use of tattoos for victim and suspect identification in forensics and law enforcement agencies such a system will be of great value in apprehending suspects and identifying victims. They introduced image similarity computation based on SIFT features [2]. Demographic information such as gender, ethnicity and facial marks as the soft biometrics traits. They leveled the gender of each face images into three categories - male, female, and unknown as well as ethnicity into three categories - Caucasian, African-American, and unknown. The author currently leveled the gender and ethnicity as a manual. They proposed a fully automated facial marks extraction system using global and local analysis method. First they applied Active Appearance Model to detect and remove the primary facial feature from face image using PCA algorithm such as eye, eyebrows, nose and mouth. The local irregularities are detected using Laplacian of Gaussian (LoG) or Difference of Gaussian (DoG). The 
detected facial marks are used to calculate by their morphology and color along with the location. They developed a soft biometric trait-based face matching system. It uses gender and ethnicity information and facial marks. This soft biometric matcher can be combined with any facial matcher to improve the recognition accuracy or used by itself when a face matcher fails because of face occlusion. The author also showed that facial marks can help in discriminating identical twins. With the proposed soft biometric matcher, users can issue semantic queries to retrieve images of interest from a large database [1]. Facial skin exhibits various small scale structures in the surface (wrinkles, scars) and the texture (nevi - a general term for pigment lesions like birthmarks and moles) that stands out from the normal skin appearance and represent potentially valuable references for individual distinction. Among such skin irregularities moles are especially suited for identification. Their predictable appearance, also under changing illumination, facilitates detection. And their numerous appearance in conjunction with unique distribution patterns scales well with extensive galleries. Furthermore moles require no abstract encoding, in contrast to most other facial features. The goal of their paper is to present techniques for detection and validation of moles that are prominent enough to be used for identification. Relying on such small scale variations is an unusual approach in face recognition. Conventional recognition algorithms are designed to work on low resolution images. J. S. Pierrard and T. Vetter et al. (2007) introduces a novel framework capable of detecting prominent moles. They attach importance to the fact that, in contrast to existing methods are not bound to a certain recognition scenario. That means in particular that they worked independent of pose and illumination and that they are able to deduce personally identifying features from one gallery image per individual. Their systems are divided into three main steps corresponding to the three properties that characterize a local region as a birthmark namely appearance, location and context. From distance a mole appears simply as small dark region of circular shape surrounded by a brighter area, i.e. a so called 'blob'. This description also holds under varying 
viewing conditions (pose/illumination). A very sensitive multi scale detection scheme to identify even the most subtle mole is also discussed. Due to its sensitivity, the detector also responds to typical facial features such as nostrils, corners of eyes eyebrows and mouth as well as to unexpected deviations like strands of hair. Across individuals, these points are not discriminate and it is crucial for their scheme that they are rejected. With prior knowledge derived from the Morphable Model, they compute a binary segmentation of the face in order to rule out points in those non-skin areas. In contrast to most other skin a detection/segmentation scheme our approach is texture based and therefore requires no color input or training. Finally the notion of saliency was introduced which allows to assess the importance of each birthmark candidate for recognition. This procedure takes the relationship between a point's size and contrast and the texture of its neighborhood into account. In essence it represents a combined measure of confidence and uniqueness. Below a certain saliency threshold, points are immediately discarded. Their framework incorporates face-class specific knowledge from the 3D Morphable Model developed by Blanz and Vetter. The model is derived from 3D laser scans, registered to a common reference in which shape and texture are parameterized. A statistical analysis of this data is performed by Principal Component Analysis. The resulting sets of orthogonal eigenvectors represent facial prototypes which can be linearly combined to form new faces. For a set of model parameters (linear coefficients) and additional parameters for pose and illumination, a realistic face image can be synthesized. Given a photograph of a face, an analysis-by-synthesis approach is used to fit these parameters such that the rendered face model matches the input image. The result is a complete $3 \mathrm{D}$ reconstruction of the face where shape and texture in occluded regions (due to pose) have been automatically replenished from the model. They detect moles by means of normalized cross correlation (NCC) matching. As a template, a Laplacian-of-Gaussian filter mask is used, since it is very much similar to the blob-like appearance of moles. The NCC has not scaled invariant and the object 
size is not known a priori. This requires computation of the matching for several resolutions, using templates of varying scale. With a growing number of resolutions a straightforward implementation becomes very inefficient. Their paper presented a novel approach to exploit local skin irregularities as features for face identification. They focused on the methodology of detection and evaluation of such regions and showed that it is possible to determine a person's identity based on only a few well-chosen pixels [5]. D. Lin and X. Tang et al. (2006) introduced a multi layer framework for high resolution face recognition exploiting features in various scales. Each face image is factorized into four layers: facial organs, the appearance of global, skins, and details of irregularities. They employed Multilevel PCA followed by the Regularized LDA to model global appearance and organs of the face. But, the description of texture of the skin and details of irregularities, for which representation of conventional vector are not appropriate. For this problem, Discriminative Multiscale Texton Features and SIFT- Activated Pictorial Structure are proposed to describe the skin and details of subtle respectively. In order to combine the information effectively conveyed by all layers, they further designed a metric fusion algorithm adaptively placing emphasis onto the highly confident layers. They established a novel and effective paradigm for high resolution face recognition, incorporating new representations for modeling skin texture and irregular details and a flexible fusion scheme [6]. We call a face recognition method as Digital Image Skin Correlation, DISC, which is based on a feature tracking method. Unlike other feature tracking methods that require a set of sparse markers to be attached to the actor's face or have a sparse set of points, the natural texture of the skin for tracking the motions of the face is used by DISC. The distribution of pores of skin on the face provides a highly dense texture, which they exploit for capturing fine-scale facial movements. By tracking an individual's skin pores during a facial expression, such as a subtle smile, they can generate a vector field that describes the individual's facial movement. They refer to these vector fields as facial deformation fields. Since facial movements are comprised of a 
complex sequence of muscle actions, which are unique to an individual, it is almost impossible to imitate an individual's facial expression. This suggests that they can use the facial deformation fields for face recognition. S. Pamudurthy, E. Guan, K. Mueller, and M. Rafailovich et al. (2005) said their method leverages the strengths of both still-image and video-based methods, using high-resolution images to extract temporal information. An important feature of their method is that it is completely based on affordable mainstream technologies. The only hardware they require is a high-resolution digital camera. In their experiments, they had observed that a digital camera with resolution greater than 4 mega pixels is sufficient to capture images that enable us to accurately track the skin pores on an individual's face. Digital Image Skin Correlation (DISC) is based on a material science technique called the Digital Image Speckle Correlation. DISC analyzes two images taken before and after the skin has deformed and yields a vector field corresponding to the skin deformation. Displacement vectors are derived by the DISC by tracking pores of the skin in the digital images before and after deformation. In order to recognize an individual, they capture two highresolution images of the subject's face - one with a neutral expression and the other with a subtle smile. They used DISC to compute the deformation field that represents the way the subject's deforms of face. Then, they generate two scalar fields from each vector field, instead of directly using the field of deformation as the feature for recognition, which are then used in the recognition process. They call these scalar fields' projection images since they are obtained by projecting each vector in the deformation field onto the $\mathrm{X}$ - and $\mathrm{Y}$-axes, respectively. Alternatively, they also separated the deformation field into magnitude and orientation fields, and possibly used their gradients as features. In order to validate the effectiveness of their framework and test its robustness, they compared the ability of their approach with that of still-image-based approaches in the task of recognizing people with heavy makeup. For this purpose, two small databases have been created since the standard recognition of the face databases does not contain the kind of images and tracking data 
they require. To compare their approach to still-image-based recognition, they used the PCA+LDA algorithm from the CSU Face Identification Evaluation System (CSU Faceld Eval) method as their baseline algorithm. The author contributed a novel method for face recognition that is based on tracking the dense texture of skin pores naturally present on the face instead of placing cumbersome and less dense markers on the person's face. Their approach combines the strengths of both the still-image methods and video-based methods [7].

\subsection{Demographic based authentication}

To verify the faces they used two methods first method namely; "attribute" classifiers - uses binary classifiers trained to recognize the presence or absence

of describable aspects of visual appearance (e.g., gender, race, and age). And second method - "simile" classifiers - removes the manual labeling required for attribute classification and instead learns the faces similarity, or faces regions to particular reference people. In this paper they attempt to advance the state-ofthe art for face verification in uncontrolled settings with no cooperative subjects. To this end, they present two novel and complementary methods for verification of face. The idea of extracting and comparing "high-level" traits or visual features, of an image of the face that are not sensitive to pose, expression, illumination, and other imaging conditions which are common to both methods. Their approach for face verification does not use expensive computation to align pairs of faces. The relatively short (65-3000 dimensional) vector of outputs from the trait classifiers (attribute and simile) are computed on each face independently. Comparing two faces is simply a matter of comparing these trait vectors. They introduced Attribute classifier, Smile classifiers, Pub Fig data set and Human Performance Evaluation. Also they used to perform face verification on a pair of images; they compare the score of both images. In their method number of steps are involved: Extract low level features, Compute visual traits, and Perform Verification. The author presented and evaluated two approaches 
for face verification using traits computed on face images - based on describable attributes and novel simile classifiers. N. Kumar, A. C. Berg, P. N. Belhumeur, and S. K. Nayar et al. (2009) contributed this was the first time such attributes have been applied to face verification [4]. Utilization of soft biometrics traits like height, age ,gender, weight, and ethnicity to complement the identity information provided by the primary biometric identifiers. The biometric recognition system is divided into two subsystems. One subsystem is called the primary biometric system and it is based on traditional biometric identifiers like face, hand-geometry and fingerprint. The second subsystem which is also known as the secondary system of biometric is based on the traits of soft biometric like age, gender, and height. The author contributed utilization of ancillary user information like gender, height, and performance of the traditional biometric systems like fingerprint can be improved by the ethnicity. Although these soft biometric characteristics are not as permanent and reliable as the traditional biometric identifiers like a fingerprint, they provide some information about the identity of the user that leads to higher accuracy in establishing the user identity [3]. To improve the Forensic Examination, the FBI also preferred Forensic Audio, Video and Image Analysis Unit (FAVIAU) perform forensic examinations such as human identification (from image and video) including comparison of faces, comparison of ears, comparison of hands, and determination of height. The author conducted two types of examination to involve biometrics performed by image examiners within FAVIAU- photographic comparisons and photogrammetry. In both of these examinations, images depicting the subject, i.e. the 'questioned individual', are submitted to the FBI for analyses. The photographic comparisons of peoples are generally one to one comparisons of person questioned and unknown. The goal of photographic comparisons is to determine if the questioned individual is the suspect. If the questioned individual and the unknown are identical, the process is known as individualization or identification in forensic community. The other types of photogrammetry are the indirect determination of special measurements of the 
object using photographic image. The main objective of photogrammetry is to determine the height of a questioned individual or to determine the length of a weapon used in the commission of a crime. This also involves two methods reserve projection or analytical photogrammetry. Height determination is commonly done to eliminate the suspect or to link multiple crimes, because human height follows a Gaussian distribution and thus can rarely used for identification of a person [10].

\subsection{Different face recognition methods}

A local feature based method for matching facial sketch images to photographs of faces was used for performing such matching and this is the first known feature based method. Starting with a sketch training set of correspondences of the photo such as a set of sketch and photo images of the same subjects, B. Klare and A. K. Jain et al. (2010) demonstrated the ability to match the sketch photos directly using SIFT feature descriptors. Their method measures the similarity between a sketch and a photo by their distance from the training set of sketch or photo pairs. They first computed using SIFT feature representation for each sketch query, gallery photograph and each sketch or photo correspondence to the dictionary. The author contributed that their proposed sketch or photo matching method is an effective than others. Their method uses local image feature to describe both sketch and photo images in a common representation framework [8]. The facial biometrics community is currently focused on developing new algorithms or attempting to fuse biometrics. The simple solution to enhance the accuracy of automated systems right now is to utilize human subject matter experts in the procedure. Every person contrasting facial images will be trained to the highest possible level of knowledge and practice in their field. The human face is effectively an elastic sculpture built upon the foundation of bones and muscles, wrapped in skin, and subject to change with expression, age, and damage. The specifics that need to be included in a training program are the properties of the face, the alteration of the 
face, and the methods of comparing faces. The author elaborated that all facial images should be trained in the structure of the bones and muscles of the head. Training should include a link between external expressions and the internal muscle groups involved in making such expressions, in order for the examiner to compare images depicting differing facial expressions. Furthermore, because wrinkles are typically formed perpendicular to the direction of muscle extension, knowledge of the muscles of the head is important to understanding the aging of the face. The outermost layer of the face, the skin, bears the texture that is most critical in individualizations. Commonly it is the co-location of facial blemishes, marks, and patterns thereof that allow a facial image examiner to identify a person to the exclusion of all others. Also they pay attention to the characteristics of the ear. N. A. Spaun et al. (2009) also mentioned the drawbacks to facial biometrics since the face is not stable over the life and aging of a face is the most common alteration depicted [9]. For recognition of human faces from single images out of a large database containing one image for each person, faces are represented by labeled graphs based on Gabor wavelet transform. Image graphs of new faces are extracted by an elastic graph matching process and can be compared by a simple similarity function. The author made three major extensions to this system; firstly they used the phase of the complex Gabor wavelet coefficient to achieve more accurate location of the nodes and to disambiguate patterns which would be similar in their coefficient magnitudes. Secondly, they employed object adapted graphs, where the nodes refer to specific facial landmarks called fiducial points. Across large viewpoint changes, the correct correspondences between two faces can then be found. Thirdly, they had introduced a new data structure, called the bunch graph, which serves as a generalized representation of faces by combining jets of a small set of individual faces. This allows the system to find the fiducial points in one matching process, which eliminates the need for matching each model graph individually. This reduces computational effort significantly. A first set of graphs is generated manually. Nodes are located at fiducial points and 
edges between the nodes as well as correspondences between nodes of different poses are defined. Once the system has an FBG (possibly consisting of only one manually defined model), graphs for new images can be generated automatically by elastic bunch graph matching. Initially, when the FBG contains only some faces, it is required to correct and review the resulting matches, but once the FBG is rich enough (approximately 70 graphs) one can rely on the matching and generate large galleries of model graphs automatically. Matching a FBG on a new image is done by maximizing a graph similarity between an image graph and the FBG of identical pose. It depends on the jet similarities and a topography term, which takes into account the distortion of the image grid relative to the FBG grid. The author had applied it to face recognition. They also tested the system with respect to rotation in depth and differences in facial expression. They did not investigate robustness to other variations, such as illumination changes or structured background [11]. A new approach for face recognition one that is insensitive to large variations in lighting and facial expressions. P. N. Belhumeur, J. P. Hespanha, and D. J. Kriegman et al. (1997) noted that lighting variability contains intensity and also the number and direction of light sources. Their approach to face recognition exploits two observations: a) All of the images of a surface of Lambertian, which are taken from a fixed viewpoint that are under varying illumination, lie in a linear subspace of 3D of the high-dimensional image space. b) Because of regions of specularities, shadowing and expressions of the faces, the above observation does not exactly right. In practice, certain facial regions may have varied from image to image that often deviates significantly from the linear subspace and consequently are less reliable for face recognition. They made use of these observations by finding a linear projection of the faces from the high-dimensional image space to a significantly lower dimensional feature space which is insensitive both to variation in lighting direction and facial expression. They considered projection directions that are nearly orthogonal to the within-class scatter, projecting away variations in lighting and facial expression while 
maintaining discriminability. Their method 'fisherfaces' which is a derivative of Fisher's Linear Discriminant (FLD) maximizes the ratio of between-class scatter to that of within-class scatter. The experiments suggest a number of conclusions: a) all methods perform well if presented with an image in the test set which is similar to an image in the training set. b) The 'fisherface' method appears to be the best at extrapolating and interpolating over lighting variation, even though the Linear Subspace method is a close second. c) Removing the largest three principal components does improve the performance of the Eigenface method in the presence of lighting variation, but does not achieve error rates as low as some of the other methods described here. d) In the limit, as more principal components are used in the Eigenface method, performance approaches that of correlation method. Similarly performance improves as the dimensionality of the feature space is increased when the first three principal components have been taken out. However, that performance seems to level off at about 45 components of the principal. A similar point of diminishing returns is found out by Sirovitch and Kirby when using Eigenfaces to represent face images. e) The Fisherface method appears to be the best at simultaneously handling variation in lighting and expression. The Linear Subspace method suffers when confronted with a variation in facial expression as expected [12]. Another way appearance-based face recognition method is the Laplacianface approach [13]. The images of face are mapped into a subspace of face for analysis by using Locality Preserving Projections (LPP). Unlike from Principal Component Analysis i.e. PCA and Linear Discriminant Analysis i.e. LDA, where the Euclidean structure of the face space is seen effectively, an embedding is found out by LPP where local information is preserved, and a face subspace is obtained and the essential face manifold structure is detected at best. The faces of the Laplacian are the minimal linear approximations to the Eigen functions of the Laplace Beltrami operator on the manifold of face. They showed how PCA, LDA, and LPP arise from the same principle applied to different choices of this graph structure. Author illustrated that while the Eigenfaces method aims to 
preserve the global structure of the space of images, and the Fisherfaces method aims to preserve the discriminating information; the Laplacianfaces method aims to preserve the local structure of the space of the image. The local manifold structure is more significant than the global in many real-world classification problems, especially when nearest-neighbor like classifiers, Euclidean structure is used for classification. Although it is unsupervised, LPP seems to have power of the discrimination. An efficient subspace learning algorithm for face recognition should be able to discover the nonlinear manifold structure of the face space. Their Laplacianfaces method which is proposed to explicitly also consider the manifold structure which is modeled by an adjacency graph. Moreover, by finding the optimal linear approximations to the Eigen functions of the Laplace Beltrami operator, the Laplacian faces are obtained on the face manifold. By finding the optimal linear approximations to the eigenfunctions of the Laplace Beltrami operator on the manifold, LPP is obtained. LLE is nonlinear, while LPP is linear. Moreover, LLE is defined only on the training data points while LPP is defined everywhere, and it is not clear how to evaluate the maps for recent test points. In contrast, to locate it in the reduced representation space, LPP may be simply applied to any new data point. The manifold ways of face analysis (representation and recognition) are introduced in that paper in order to detect the underlying nonlinear manifold structure in the manner of linear subspace learning [13]. P. S. Penev and J. J. Atick et al. (1996) constructs, from the global PCA modes, a local topographic representation of objects in terms of local features. The procedure, which they call local feature analysis i.e. LFA, a dense set of local feed forward is derived in a receptive field, defined at each point of the grid of receptor and different from each other, that is optimally matched to the input ensemble, and whose outputs are as decorrelated as possible. Since the objects from the input ensemble span only a very-low-dimensional subspace, the dense set of outputs necessarily contains residual correlations. They used these residual correlations to define lateral inhibition which acts to specify the output. Thus, the final representation is a 
local sparse-distributed representation in which only a small number of outputs are active for any given input. The number of active units is of the order of the dimensionality of the PCA subspace, but their subset changes from one input example to another, providing valuable additional information about the locations of the features in the currently represented example. They gave a practical implementation for finding approximations to the stable states of this network dynamics that is computationally very efficient on a serial machine. They illustrate LFA by using it to derive local features in three different object ensembles that are similar to ensembles for which global representations have been derived previously. The first ensemble 2D images of faces without background serves to illustrate the ability of the method to derive local features intrinsic to objects; it yields receptive fields for noses, mouths, eyes, cheeks, etc. The second ensemble 3D surfaces of human heads while producing the same conceptual features, has the added advantage of being much more controlled since it contains no variability due to scale, light or albedo in the data. In this case we derive high-quality 3D filters that are matched to the 3D features. The third ensemble comprises of $2 \mathrm{D}$ images of faces on a background. Besides the regular 'face' features, the most prominent additional ones that LFA derives, are those signaling transitions from the background to the head. The positions of activation provide an accurate outline of the head that could subsequently be used for segmentation. In their paper they had shown how to overcome the main limitation of the PCA representation by developing LFA. A method for deriving a dense set of local topographic feed forward receptive fields that are defined at each point of the receptor grid, different from each other, optimally matched to the second-order statistics of the input ensemble, and having outputs as decorrelated as possible. Then they used the residual correlations to define lateral inhibition which specifies the output, recovering the low dimensionality, and further decorrelating the representation. The resulting receptive fields are matched to the structures that are expected at their respective centers, thus giving a representation of the objects in terms of their local features [14]. A new 
unsupervised nonlinear feature extraction method is called spectral feature analysis i.e. SFA. The advantages of SFA over traditional feature extraction methods are: (1) SFA does not suffer from the small-sample-size problem; (2) SFA can extract discriminatory information from the data and that linear discriminant analysis which can be subsumed under the SFA framework is shown; (3) SFA can effectively discover the nonlinear structure that are hidden in the data. For face recognition tasks, these appealing properties make SFA very suitable. Their work here is motivated by the recent works on spectral clustering, which is a class of clustering methods based on the spectral graph theory. These clustering methods usually take two steps. First, the patterns are embedded in a lower-dimensional space such that the clusters are more "obvious" or separate. Then, a classical clustering algorithm (such as the $K$ means clustering algorithm) is performed in the embedded space. Spectral clustering can yield impressively good results when traditional clustering approaches fail, and it has been shown to be very powerful in fields such as computer vision and VLSI design. They presented in their paper a related unsupervised feature extraction method called spectral feature analysis (SFA). The embedded data resulting from the first step of spectral clustering will be referred to as spectral features. Since spectral clustering can only compute spectral features for the training set, a natural question is how to obtain spectral features for the unseen (i.e., testing) data. To address this problem, they first proposed a weighted extension of the standard kernel principal component analysis (KPCA) called weighted kernel principal component analysis (WKPCA). Then, they showed that the spectral clustering is a special case of WKPCA and, consequently, spectral features of the test set can be obtained by WKPCA. Their method is particularly suitable for face recognition because of the following properties: SFA does not suffer from the small-sample-size problem. In both LDA and LPP, the resultant computational problems involve generalized eigenvalue problems, which in turn require matrix inversions. The sizes of the matrices involved, depending on the dimensionality of the image, which are 
often very large compared to the number of training images. These matrices may thus be singular and the small sample-size problem occurs. On the other hand, although the resultant computational problem in SFA is still a generalized Eigenvalue problem, the size of the matrix that has to be inverted is only dependent on the number of training patterns. As spectral clustering can effectively group patterns into clusters, SFA can also effectively extract discriminative information from the data. Furthermore, they will prove that LDA can be subsumed under the SFA framework. SFA can effectively discover the intrinsic nonlinear manifold structure hidden in the data. Indeed, they will prove that kernel LPP is SFA. In other words, their method can exploit and preserve the data's nonlinear manifold structure [15]. Also describes image features that have many properties that make them suitable for a scene or matching differing images of an object. To image scaling and rotation, the features are invariant and partially invariant to change in illumination and 3D camera viewpoint. In both the spatial and frequency domains, they are well localized, reducing the probability of disruption by occlusion, clutter, or noise. With efficient algorithms, large numbers of features can be extracted from typical images. Additionally, the features are highly different, that allows a single feature to be correctly matched with high probability against a large feature of the database, giving a basis for object and recognition of scenes. The extracting cost of these features is minimized by taking a cascade filtering approach, in which the more expensive operations are applied only at locations that pass an initial test. There are the major stages of computation used to generate the set of features of image: 1. Space of scale detection of extrema: The first stage of computation searches over all scales and locations of image. To identify potential interest points that are invariant to scale and orientation, it is implemented efficiently by using a difference-of-Gaussian function. 2. Localization of keypoint: A detailed model is fit to determine the location and scale at each location of the candidate. Based on measures of their stability, key points are selected. 3. Assignment of orientation: One or more orientations are assigned to each keypoint location 
based on local directions of image gradient. In image data, all operations of the future are performed which has been transformed relative to the assigned orientation, location and scale for each feature. As a result, invariance is provided to these transformations. 4. Descriptor of Keypoint: At the selected scale in the region around each keypoint, the local image gradients are measured in which it is transformed into a representation which allows for significant levels of local shape distortion and change in illumination. This method is named as the Scale Invariant Feature Transform i.e.SIFT, as it converts image data into scale-invariant coordinates relative to features of local. From a set of reference images, SIFT features are first extracted and stored in a database for image matching and recognition. By comparing individually each feature from the new image to this previous database and finding candidate matching features based on Euclidean distance of their feature vectors, a new image is matched. Due to their distinctiveness, the SIFT keypoints described in their paper are particularly useful which enables to be selected from a large database of other keypoints and gives the correct match for a keypoint. By assembling a high-dimensional vector representing the image gradients within a local region of the image, this distinctiveness is achieved. The keypoints have been shown to be invariant to rotation of the image and scale and robust affine distortion across a substantial range, noise addition, and illumination change. From typical images, large numbers of keypoints can be extracted, which result to the robustness among the clutter in extracting small objects. The keypoints are detected over a complete range of scales and small local features are available for matching objects which are occluded small and highly, while for images subject to noise and blur, large keypoints performed well. Their paper has also presented methods for using the keypoints for object recognition [16]. To extract the feature most powerful algorithm SURF (Speed-Up Robust Features) was introduced in the year 2006. The author claimed that his algorithm is more powerful than SIFT algorithm. They used SURF as feature extraction corresponding points between two images of the same scene or 
object of many computer vision applications. The search between two corresponding points can be divided into three steps. First, 'interest points' are selected at distinctive locations in the image, for instance corners, T-junctions and blobs. Repeatability is the most valuable property of an interest point detector. The reliability of a detector is expressed by the repeatability for finding the same physical interest points under different viewing conditions. Secondly, a feature vector represents the neighborhood of every interest point. This descriptor must be robust to noise, displacements of detection and geometric and deformations of photometric and at the same time, it has to be distinctive. Finally between different images, the descriptor vectors are matched. Based on a distance between the vectors, the matching is done. Also they focused on the scale and in plane rotation invariant detectors and descriptors. Common issue between feature complexity and robustness to commonly occurring photometric deformations offered by it was studied. Perspective effects, anisotropic scaling and skew are assumed to be second order effects that are covered to some degree by the overall robustness of the descriptor. The descriptor can be extended towards affine invariant regions using affine normalization of the ellipse. Their work involved mainly on the interest point detection scheme and new descriptor. They used Hessian matrix detection scheme because of its good performance in computation time and accuracy. Their proposed SURF had more fast and performance interest point detection-description scheme which outperforms the current state of the art, both in speed and accuracy claimed by the author [26]. Another survey conducted recently on Speed up robust feature detection explores the reduction of the scale space "Octaves" as a key speed improvement. Rather than simply discarding the higher octaves, which may reduce accuracy, their paper developed a more sophisticated approach, which is termed as best octaves, that examines all octaves and then chooses two octaves that provide the best matching performance according to their argument. The author demonstrated that the best octaves outperform SURF variants obtained using the convolutional approach. They claimed that 
this was the first systematic approach to SURF octave section and the time complexity is reduced [27]. The use of multiple sensors addresses the problem of noisy sensor data, but all other potential problems associated with unimodal biometric systems remain. A recognition system that works on multiple instances of the same biometric can ensure the presence of a live user by asking the user to provide a random subset of biometric measurements. A multimodal biometric system based on different traits is expected to be more prone to noise, the problem of non-universality is addressed, the matching accuracy is improved, and reasonable protection is provided against spoof attacks. Therefore, based on multiple biometric traits the development of biometric systems has received considerable attention from researchers. In a multimodal biometric system that uses different traits of biometric, various fusion levels are possible: fusion at the feature level of extraction, matching the level of score or level of decision. It is difficult to consolidate information at the feature level because the feature sets used by different biometric modalities may either be inaccessible or not compatible. At the decision level, fusion is too rigid since only a limited amount of information is available at this level. Therefore, integration at the matching score level is generally preferred to the ease in accessing and combining matching scores. Given the scores of different modalities, they used the combination approach to fusion and address some of the issues involved in computing a single matching score. Normalization is required to transform these scores into a common domain before combining them as the matching scores generated by the different modalities are heterogeneous. There has been no detailed study of these techniques while several normalization techniques have been proposed. Based on the face, fingerprint and hand -geometry modalities, in this work, they have systematically studied the effects of different normalization schemes on the performance of a multimodal biometric system. The author examined the effect of different score normalization techniques on the performance of a multi model biometric system and demonstrated that there is a considerable improvement in the recognition performance of a multi model 
biometric system that uses the face, fingerprint and hand geometry traits for user authentication [22].

\subsection{Face annotation methods}

Investigations are also growing on new user scenario for face annotation in which users are allowed to multi select a group of photographs and assign names to that photograph. Then the system will attempt to propagate names from photograph level to infer the correspondence between faces and images from photographs. The authors measured the facial similarity using thumbnails to see who is in each photograph uses his/her mouse to multi select all of the photographs with a particular individual that he/she wants to level. This interface further reduces the users labeling efforts because the function of browsing thumbnails becomes common in most photograph management system, and the user works on photograph level, instead of face level. Thus users can level the group of photographs in a batch way. Also the author derived the objective function as the sum of similarities between each pair of face of the same individual in different photographs. Maximization of the objective function leads to the optimal solution for name propagation. The author further takes advantages from user's interactive operation between annotation and propagation to make the system more effective in annotation and propagation. This means that the name propagation accuracy can be improved if some faces have annotated before, and similarly, the annotation accuracy can be improved if some photographs have been associated with names [17]. A novel face annotation method that systematically and automatically combines (or leverages) context information about time and space (or background) of personal photos with conventional FR technologies. In their proposed method, traditional FR techniques can take benefits to improve annotation accuracy by taking into account context information. In addition, their method requires no training data labeled by hand from photos in contrast to previous research in this field. Practically, it is highly desirable in most cases with a shortage of labeled 
data. Their proposed method largely consists of three steps: situation clustering based on contextual information, person clustering with an efficient fusion of person identity features, and face recognition for the purpose of annotating faces on personal photos. Using context-based situation and person clustering, they obtained a useful group, called 'person cluster', of multiple facial images that belong to the same person. In the practical FR applications, they may be varied significantly due to practical problems like changes in illumination and pose, although facial traits are extracted from the same person. To overcome those limitations by combining their multiple evidences, multiple facial images of the same person are used for FR methods. Multiple facial images included in each person cluster are to be annotated by conventional FR methods in conjunction with effective information fusion strategies. Three representative subspace FR methods are adopted as a FR framework in the following: Principal Component Analysis i.e. PCA or "eigenfaces", Fisher-Linear Discriminant Analysis i.e. FLDA or "fisher-faces, and Bayesian i.e. "Probabilistic eigenspace". To efficiently take advantages of multiple facial features per person, feature and measurement level fusion strategies are used. To demonstrate the propose of face annotation approach, they conducted extensive experiments with personal photos used in the standard MPEG-7 VCE-3. The authors concluded that their method provides new insights for efficient integration of contextual information of multimedia contents with computer vision technologies [18]. Face detection and recognition have numerous multimedia applications of wide interest, in which one is automatic face annotation. A real-time face annotation system implemented on a cellular-phone-based embedded platform. They developed the face detector based on the OpenCV face detector. This detection method, originally proposed by Viola and Jones, (2001) is one of the most widely used algorithms and can achieve a great detection rate with fast computation. This method applies a cascade classifier structure that consists of a series of stages. Each stage consists of several weak classifiers that can quickly reject non-face regions so more computational resources are reserved to examine more 
promising, face-like regions. For the face recognition purpose the author used ARENA algorithm. Also they used ARENA for comparing all pixel values of the detected face to those of images in the training set [19].

\subsection{Facial feature detection algorithms}

To detect a facial feature used Active appearance model (AAM). An AAM contains a statistical model of the shape and the gray level appearance of the object of interest. The approach follows the method described in Edwards et al. (1996) but include extra normalization and weighting steps. The models were generated by combining a model of shape variation with a model of the appearance variation in a shape normalized frame. The collected training set of images which are labeled, where on each example object, key landmark points are marked. For instance to build a face model we need face images marked with points at key position to outline the main feature. Given such a set, they generated a statistical model of shape variation. The labeled points on a single object describe the shape of that object. Then the set is aligned into a common coordinate frame which is represented by a vector $\mathrm{x}$. The Principal Component Analysis (PCA) is applied to the data. To build the statistical model of the grey level appearance they warp each example image so that its control points match the mean shape using triangulation algorithm. They used training set of 400 images of faces, each labeled 122 points around the main features. From that they generated a shape model with 23 parameters. To new images, they demonstrated an iterative scheme for fitting an Active Appearance Model (AAM). This method makes use of learned correlation between model displacement and the resulting difference image [20]. For various image analysis and synthesis problems, Active Appearance Model (AAM) framework is a very useful method that can fit the shape and appearance model to the input image. However, it often fails to accurately converge to the landmark points of the input image since the goal of the AAM fitting algorithm is to minimize the residual error between the model appearance and the input image. To alleviate this weakness, They 
combined Active Shape Models i.e. ASM into AAMs, by using the local profile model, to find correct landmark points. They derived a gradient based iterative method by modifying the objective function of the ASM search since the original objective function of the ASM search is not appropriate for combining these methods. They proposed a new fitting method that combines the objective functions of both ASM and AAM into a single objective function in a gradient based optimization framework. The proposed AAM + ASM algorithm precomputes the linear shape, appearance, and profile model independently from a set of the landmark training images and uses these models simultaneously to find the optimal model parameters for an input image. By integrating the AAM and ASM error terms and optimizing them simultaneously, we could obtain more accurate solutions than using only the AAM error term. This improvement is due to the fact that the ASM error term enforces to move the shape points to nearby edge-like points. If we only take the AAM error term, the AAM fitting often fails to converge to the ground truth points because there are no distinctive texture patterns in the cheek area of the face. One thing to note here is that the purpose of the proposed algorithm is not obtaining an illumination robustness fitting but obtaining a more accurate fitting. Experimental results show that the proposed fitting method successfully improves the fitting results in terms of root mean square (RMS) positional errors when compared to ASM, AAM, and TC-ASM [21].

\subsection{Edge detection method}

John Canny has developed a method called Canny edge detection in 1986 to detect edges. Their paper presented a method to develop a mathematical model for these two criteria which can be used to design detectors for arbitrary edges. They also discovered that the first two criteria are not "tight" enough, and that it is necessary to add a third criterion to circumvent the possibility of multiple responses to a single edge. To derive detectors for several common image features, including step edges, they used the criteria in numerical optimization. 
They find that there is a natural uncertainty principle between detection and localization performance on specializing the analysis to step edges, in which two main goals are there. They derive a single operator shape with this principle, which is minimal at any scale. A simple approximate implementation is there in the optimal detector in which edges are marked at maxima in gradient magnitude of a Gaussian-smoothed image. They extend this simple detector using the operators of several widths to cope with different signal-to-noise ratios in the image. For the fine-to-coarse integration of information from operators at different scales, they present a common method, called feature synthesis. They finally proved that step edge detector performance improves considerably as the operator point spread function is extended along the edge [23]. Similarly, optimal filter for edge detection has been studied by Didier Demigny et al (1997). They defined the criteria to compare the performance of different filters in their application the discrete domain. The validity of this approach is confirmed by some comparisons with experimental results. They also defined an optimal continuous filter and optimal discrete filter. They compared the performances of the sampled version of the continuous filter to those of the optimal discrete filter. They highlighted the existence of two classes of derivative filters, according to whether the impulse response in the continuous domain is continuous or not at $x$ $=0$. For a filter with a discontinuous CIR, They had computed an upper bound for the criterion of low responses multiplicity. On the other hand, criteria for better detection and low-responses multiplicity can be used with the smoothing filters. Finally, these criteria can be used to compute an optimal filter directly in the discrete domain [24]. Recently one published paper studied a region growing method, which is largely used in medical image segmentation. To improve the region growing method, they used three kinds of region growing method to segment the origin medical image namely confidence connected threshold, connected threshold and connected threshold with neighborhood. To evaluate the performance of edges locating and decide which is the most suitable, they used canny edge detection. The Canny edge detection algorithm 
is used to obtain the small edges, and then applied the edge information to compare different region growing methods to choose the suitable one. Further, they implemented image filtering and edge detection and they claimed that their method improves the small edges' locating and promotes the last segmentation result [25].

\subsection{MOTIVATION}

The face is one of the most acceptable objects for biometrics study and it has also been the most common method of recognition that human use in their visual interactions. The problem with authentication systems based on fingerprint, voice, iris and the most recent gene structure (DNA fingerprint) has been the problem of data acquisition. The method of acquiring face images is non intrusive and thus face can be used as a biometric trait for covert (where user is unaware that he is being subjected) system. Now the issue is whether faces covered with thick layer of cosmetic items to cover all scars, marks on the faces could be recognized with considerable accuracy. This type of study enhances the accuracy of face recognitions under different noises and thereby increases the level of biometric security. With this motivation a study has been done on these issues and two methods have been proposed to address several issues that arise due to layer of marks and images with low quality. The relevance of facial marks or micro features ( e.g. scars, birthmarks ) to face recognition is also presented. The main subject of this research work concentrates on two aspects (i) face recognition methods for cosmetic applied face images (ii) low quality face images.

In this thesis the main focus is to develop the face recognition system based on cosmetic applied face and to provide secure authentication in biometrics system. The proposed method is to detect the facial marks such as tattoos, scars, freckles and moles covered by cosmetic material from the face image. This method proposed gives high accuracy in facial marks detection of the cosmetic applied faces. In fact the use of the robust marks detection method 
with soft biometrics traits provided the best facial recognition technology. However, there are a number of difficulties in recognizing the human face and authentication of the person perfectly. The difficulties include low quality images due to sparse dark or light disturbances. To overcome such kind of problems, suitable algorithms are required to filter the images and detect the face and facial marks. This technique comprises extensively of detecting the different facial marks from that of low quality images which have salt and pepper noise in them.

\subsection{THESIS CONTRIBUTIONS}

We have developed methods to improve face recognition performance in two ways: utilizing secondary features (e.g., facial marks), i) Facial marks detection from the cosmetic applied faces, ii) Facial marks detection from the low quality image. The contributions of the thesis are summarized below.

- An automatic facial mark detection method is developed that can be used in face retrieval and matching. We have applied an Active Appearance Model (AAM) [20] to localize the face boundary and mask primary facial features such as e.g., eyes, eyebrows, nose, and mouth. Canny edge detection [23] and Speed Up Robust Feature (SURF)[26] is then applied on the rest of the face area to detect facial marks.

- Facial marks detection method is developed that can be used in face retrieval and matching. We have applied an Active Appearance Model (AAM) and Active Shape Model (ASM) [21] to localize the face boundary and mask primary facial features such as e.g., eyes, eyebrows, nose, and mouth. Gradient Location Oriented Histogram (GLOH) [78] is then applied on the rest of the face area to detect facial marks. A fusion of mark based matching and a commercial matcher shows that the recognition performance can be improved. The methods used in this thesis are described below. 


\section{ROBUST FACIAL MARKS DETECTION METHOD USING AAM AND SURF}

\subsection{INTRODUCTION}

The facial marks detection provides a significant role in face recognition and matching in biometrics environment. Face recognition still remains a challenging problem to recognize the face. A number of studies are carried out to improve the face recognition performance by developing feature representation schemes. These features include the salient skin regions, which appear on the human face such as (eg. scars, moles, freckles, wrinkles, etc.) [10]. Due to improvement in image processing and computer vision algorithms, the use of facial feature has played a significant role. Local feature has a unique capability to investigate face images in the forensic application by enhancing image accuracy.

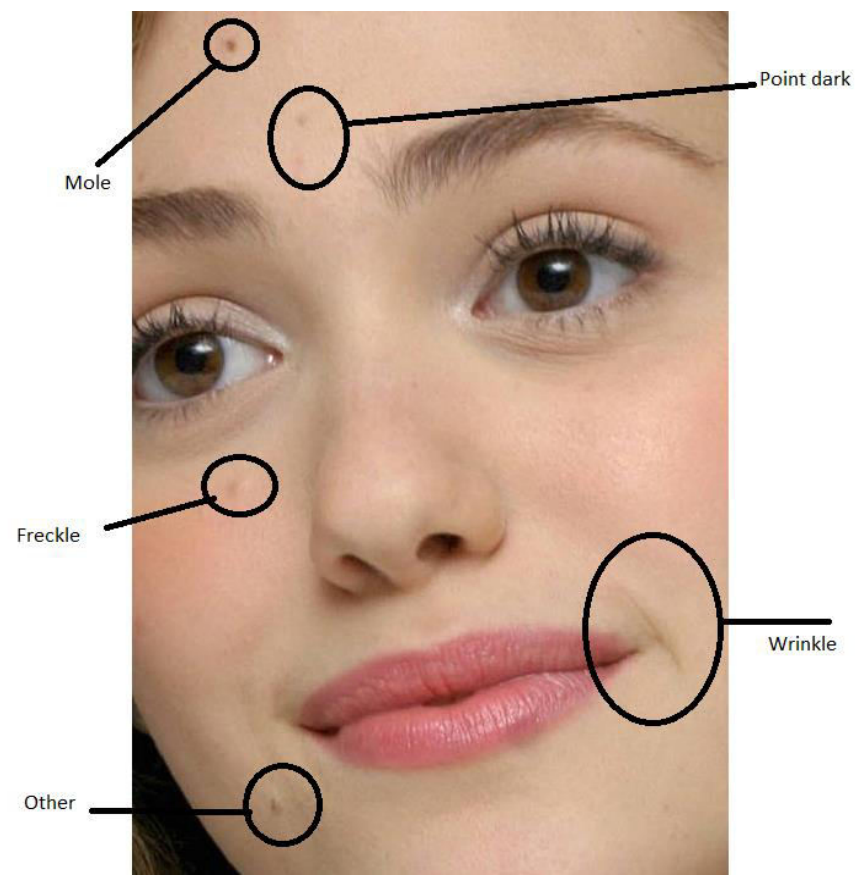

Figure 4.1, Examples of facial marks Scar, mole, and freckles etc. 
The above information is very essential to provide identification of the suspected folks for forensic experts to provide testimony [9]. Formal face recognition system encode the face images by applying either local or global texture features. Local techniques initially detect the individual components of the human face such as mouth, chin, nose, eyes etc. and prior to encoding the textural content of each of these components (e.g., EBGM and LFA) [11] [14]. In global techniques, during the face recognition process, the entire face is considered as a single entity (e.g., PCA, LDA, Laplacianfaces etc.) [12]. Example of facial marks are given in figure 4.1. Therefore, the given method has small classifiable marks on the human faces which are considered as noisy. Hence it is not used in the matching process. However, it's shown in the Face Recognition Vendor Test (FRVT 2006) [71], the face skin details such as facial marks are highly significant to attain the face recognition accuracy. Hence, facial marks can be used to filter the database to speed up the retrieval process or differentiate identical twins. Facial marks belong to a more general term, Scars, Marks, and Tattoo (SMT), which is gaining increasing attention for its utility of subject identification under non-ideal conditions for forensic investigation [72]. There have been only a few studies utilizing facial marks for the face recognition purpose [5][73]. Previous studies illustrate that facial marks are primarily focused on facial recognition performance using standard face image data set. Park and Jain [5] expressed that facial marks are very essential for identifying twins using the semi automatic concept. Also Spaun [9][10] explained that facial examination process carried out in the low enforcement agencies. One of the major examination steps involves identifying "class" and "individual" characteristics. The 'class' characteristics involve overall facial shape, presence of hair, hair color, shape of the nose, presence of marks etc. Similarly 'individual' characteristics include the number and location of scars, tattoos, location wrinkles etc in a faces. Lin at el [6] first utilized the SIFT operator [16] to extract facial irregularities and fused them with global face matcher. Facial irregularities and skin texture were used as an additional means of distinctiveness to achieve 
performance improvement. However, the individual types of facial marks not defined. Therefore the method is not suitable for face database indexing. Pierrard et al. [5] proposed a method to extract moles using normalized cross correlation method and a morphable model, they claimed that their method is pose and lighting invariant since it uses a 3D morphable model. Therefore, besides moles they did not consider other types of facial marks. Lee et al. [2] introduced "scars, marks, and tattoos (SMT)" in their tattoo image retrieval system. While tattoos can exist on any body part and are more descriptive, we are interested in marks appearing exclusively on the face which typically show simple morphologies. Pamudurthy et al. [7] used distribution of fine-scale skin marks pattern. The correlation coefficients calculated between registered skin patches are aggregated to obtain a matching score.

We completely focused on the detection of the facial marks from the face image and the hidden facial marks that are covered by cosmetic items. We use different powerful algorithms to find the facial marks from the cosmetic applied faces. Our method shows better performance contrast with the existing study. Figure 4.2, shows the mark based matcher helps in indexing each face image based on facial marks, these indices will enable fast retrieval as well as for textual or key word based query. Figure 4.3 , shows the detected facial marks in different types of faces.

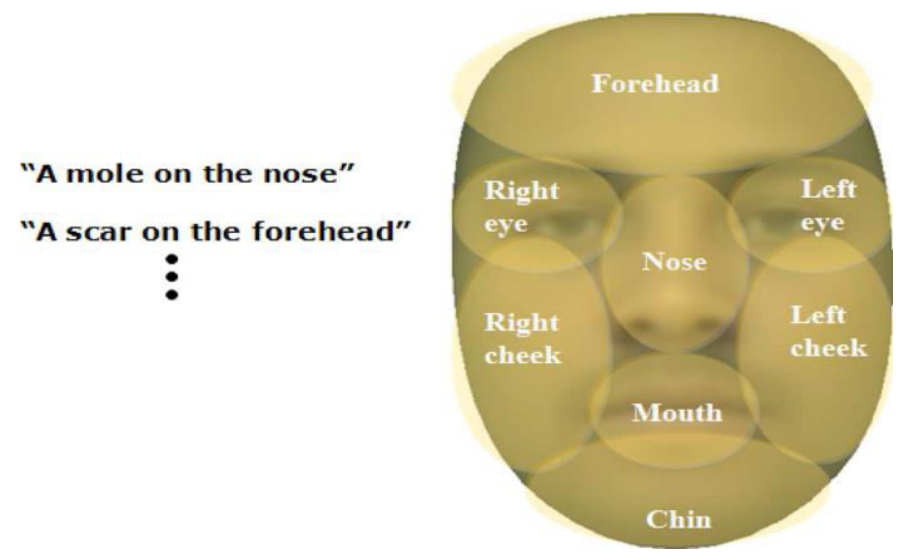

Figure 4.2, Examples of textual query and a schematic of face region segmentation. 

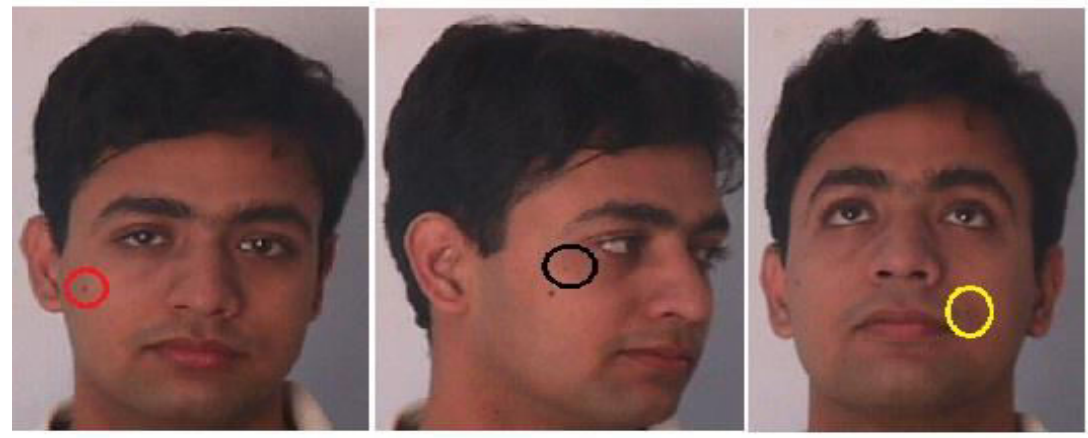

Figure 4.3, Three different types of example of facial marks results. Full face, partial face, and off-frontal face.

We initially apply the (AAM) Active Appearance Model using PCA to detect the facial features. Some facial features such as eyebrows, eyes, nose, and mouth are subtracted from the detected face image. We create a mean shape to detect the face automatically and also construct a mask for the face image. Finally, we apply the canny algorithm to identify local irregularities by detecting the edges in the image and Speed Up Robust Feature (SURF) to extract the facial features. Therefore the detected facial marks were combined to enhance the face matching accuracy. Our technique completely differs significantly from the previous studies in the following aspects: (1) initially we extract all the facial marks that are locally salient and covered by cosmetic items. (2) We concentrate on finding semantically meaningful facial marks instead of extracting texture patterns that are implicitly based on facial marks. The proposed facial marks determination concept will be helpful to forensics and law enforcement agencies because it will supplement existing facial matchers to improve the identification accuracy. 


\subsection{FACIAL MARK FINDER}

The proposed facial mark detection method is based on the appearance of salient localized regions on the face image which are covered by cosmetic items. Therefore, a feature detector SURF [26] has been applied to detect the hidden facial marks. Hence, to detect the direct facial feature on the face image it will increase the number of false positives facial marks due to presence of facial features such as eyes, eyebrows, nose, and mouth. Therefore, we initially detected the facial features of the face image and subtracted the unwanted regions to find the accurate marks present in the face, after that we extracted different facial marks present in the face image. The complete process involves different steps; the complete facial mark detection process is illustrated in Figure 4.4. We will elaborate with the following steps 1) facial feature detection 2) mean shape mapping 3) face mask construction 4) facial marks blob detection and 5) facial marks blob classification.

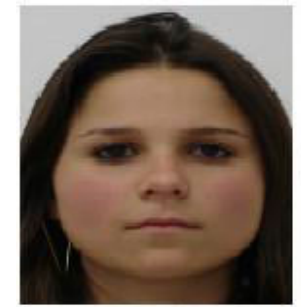

Input Image

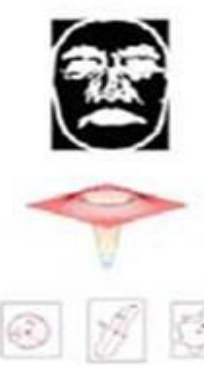

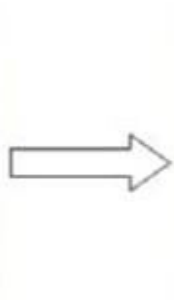

Feature point detection AAM

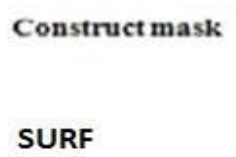

Mark classification

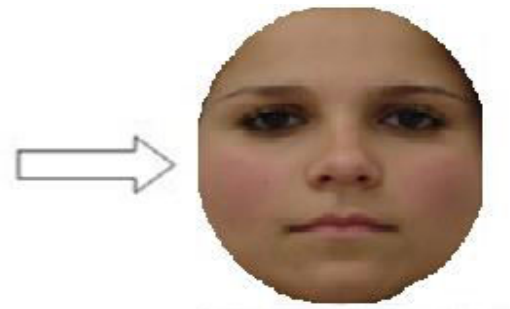

Mapping to mean shape

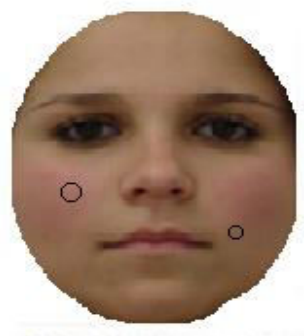

Detected face marks

Figure 4.4, Schematic of automatic facial mark extraction process. 


\subsubsection{Facial Feature Detection}

We applied Active Appearance Model (AAM) using PCA [20], [74] to detect automatically facial features with 120 landmarks that delineate the facial features such as eyes, eyebrows, nose, mouth, and face boundary (Fig. 9). Therefore the facial features detection process will be disregarded in the sequence of facial mark detection process which provides easy identification of the marks. Active Appearance Models both the shape and texture of face images using the Principal Component Analysis (PCA). We labeled manually a set of landmark points in the training data:

1.1, A set of shape information $\mathrm{X}=\left\{x_{1, \ldots} x_{\mathcal{N}}\right\}$ and corresponding textures $\mathrm{G}=$ $\left\{g_{1}, \ldots \ldots g_{\mathcal{N}}\right\}$ are obtained. Where $\left\{x_{1}, \ldots x_{\mathcal{N}}\right\}$ is the training set of aligned shapes and $\left\{g_{1, \ldots \ldots} g_{\mathcal{N}}\right\}$ is the training set of shape free normalized image patches.

1.2, Applying to get eigen value using PCA both on $X$ and $G$, the principal components of shape and texture, $\mathrm{P}_{\mathrm{x}}$ and $\mathrm{P}_{\mathrm{G}}$ are obtained. Where $\mathrm{P}_{\mathrm{x}}, \mathrm{P}_{\mathrm{G}}$ is the matrix of eigenvectors.

1.3, The image shape and texture, $x_{\text {new }}$ and $\mathcal{G}_{\text {new }}$, of a new face can be expressed as $x_{\text {new }}=x_{\mu}+\mathrm{P}_{\mathrm{x}} \mathrm{b}_{\mathrm{x}}$ and $\mathcal{G}_{\text {new }}=\mathrm{g}_{\mu}+\mathrm{P}_{\mathrm{G}} \mathrm{b}_{\mathrm{G}}$, where $\mathcal{X}_{\mu}$ and $\mathrm{g}_{\mu}\left(\mathrm{b}_{\mathrm{x}}\right.$ and $\left.\mathrm{b}_{\mathrm{G}}\right)$ are means (weight vectors) of $X$ and $G$, respectively. The 120 landmark points were labeled on a subset of database images for training.

\subsubsection{Mean Shape Mapping}

Active Appearance Model has been applied to detect the landmarks. Therefore to simplify the mark detection, we map each face image to the mean shape. Consider $S_{i}, i=1, \ldots, N$ represent the shape of each of the $N$ face images in the database (gallery) based on the 120 landmarks. Hence the mean shape is calculated using the equation $\mathrm{S}_{\mu}=\sum_{i=1}^{N} S i$. 


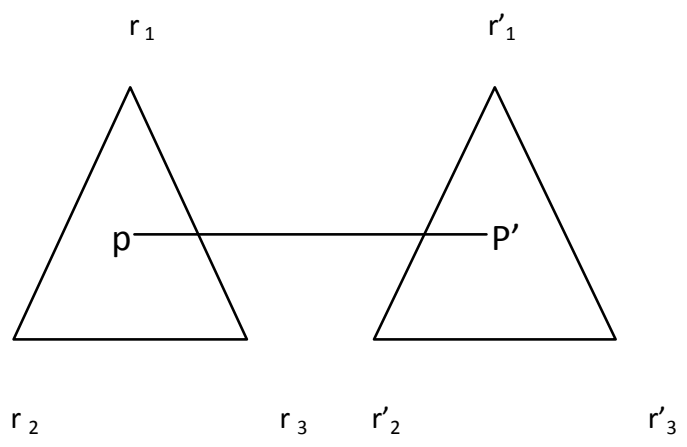

Figure 4.5, Schematic of the texture mapping process using the triangular Barycentric coordinate system.

Each face image $S_{i}$ is mapped to the mean shape $S_{\mu}$ by using the Barycentric coordinate-based [75] with the texture mapping process. Initially, both $S_{i}$ and $S_{\mu}$ are subdivided into a set of triangles. Given a triangle $T$ in $S_{i}$, its corresponding triangle $T^{\prime}$ is found in $S_{\mu}$. Let $r_{1}, r_{2}$, and $r_{3}\left(r_{1}^{\prime}, r_{2}^{\prime}\right.$ and $\left.r_{3}^{\prime}\right)$ be the three vertices of $T\left(T^{\prime}\right)$. Then, any point $p$ inside $T$ is expressed as $p=\alpha r_{1}+\beta r_{2}+$ $\gamma r_{3}$, and the corresponding point $p^{\prime}$ in $T^{\prime}$ is similarly expressed as $p^{\prime}=\alpha r_{1}^{\prime}+\beta r_{2}^{\prime}+$ $\mathrm{yr}_{3}$, where $\alpha+\beta+\gamma=1$. In this way, the pixel value is mapped and shown in Figure 4.5, using the schematic of the Barycentric mapping process. This mapping process has repeated for all the points which is present inside all the triangle, the texture in $S_{i}$ is mapped to $S_{\mu}$. Hence, after this mapping process, all face images are normalized in terms of scale and rotation and it facilitated us to represent each facial mark in a face-centered common coordinate system. Figure 4.6, shows the schematic of mean face construction. 


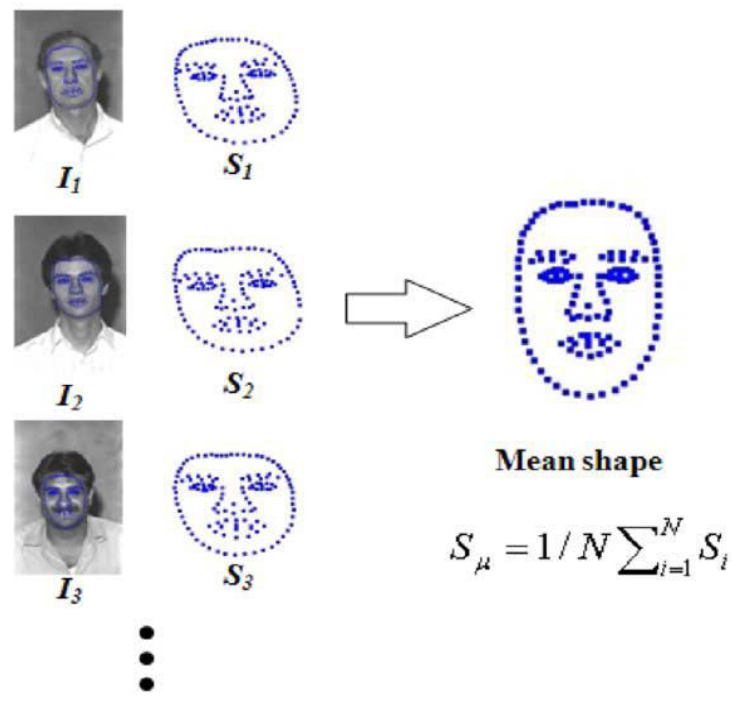

Feature point detection

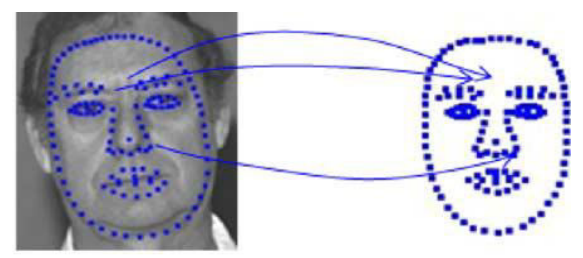

Map pixels from $S_{1}$ to $S_{\mu}$

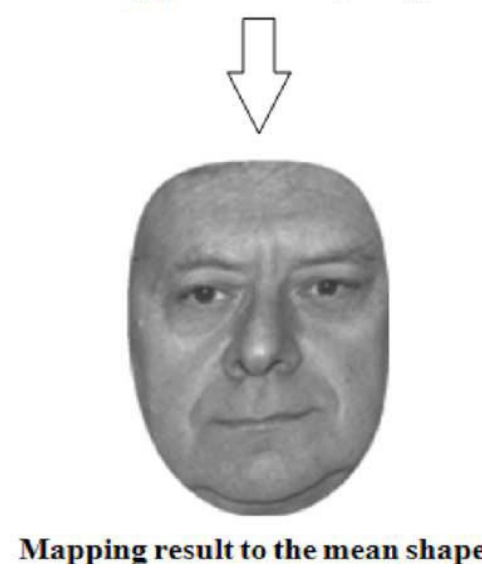

Figure 4.6, Schematic of the mean face construction.

\subsubsection{Face Mask Construction}

From the mean shape, we constructed a mask known as $S_{\mu}$ to suppress false positive values, after applying the facial feature detection for blob detection. Applying the blob detection operator to the face image mapped into the mean shape and a mask constructed from $S_{\mu}$ is used to suppress blob detection on the facial features detection process. Consider the mask constructed from the mean shape to be denoted as $\mathrm{M}_{\mathrm{g}}$, namely, a generic mask. However, the generic mask does not cover the user-specific facial features, eg. beard or small wrinkles around the eyes or mouth, that are likely to increase the false positives. Therefore we also build $\mathrm{M}_{\mathrm{s}}$ known as a user-specific mask using the edge image. Hence the conventional Canny edge detector [23][24] is applied to obtain the edge from the face image. The user specific mask $M_{s}$, constructed as a sum of $M_{g}$ and edges that are connected to $M_{g}$ is used as an aid to removing most of the false positives present around the beard or small wrinkles around eyes or mouth. 


\subsubsection{Facial Marks Blob Detection}

Our facial marks mostly appear and present in isolated blobs. To detect the facial marks which are covered such as cosmetic items, we use a familiar canny edge detector algorithm [23][24][25] and SURF [26] is applied to extract the facial marks appeared on the face. The algorithms are illustrated given below:

4.2.4.1, Canny edge detector: It was developed by John F Canny in 1986. It allows to detect the blob. Motivated by this algorithm, we detect the hidden facial marks which are covered with cosmetic items. It uses the Gaussian filter, and it introduces a two-dimensional Gaussian function, and it uses first order derivation to filter the image. The two-dimensional Gaussian function is given:

$$
\mathrm{G}(\mathrm{x}, \mathrm{y}, \sigma)=\frac{1}{2 \pi \sigma^{2}} \exp \left(-\frac{1}{2 \sigma^{2}}\left(x^{2}+y^{2}\right)\right)
$$

where $G$ is the gradient value, $\sigma$ is known as variance of Gaussian function to control the length of Gaussian windows and $x, y$ are the distance away from the target pixel. The original image denoted as $T(x, y)$ is filtered by Gaussian filter and the result is given below:

$$
F(x, y)=T(x, y)^{*} G^{\prime}(x, y, \sigma)
$$

Where $F(x, y)$ is the Gradiant function.

For the $G(x, y, \sigma)$ having two variables, the partial derivation along $x$ and $y$ direction are given : 


$$
\begin{aligned}
G_{x}(x, y, \sigma) & =\frac{\partial G(x, \mathrm{y}, \sigma)}{\partial x} \\
G_{y}(x, y, \sigma) & =\frac{\partial G(x, y, \sigma)}{\partial y}
\end{aligned}
$$

Where $G_{x}$ is the first derivative in the horizontal direction and $G_{y}$ is the vertical direction. The filtered image can be described as convolution of $T(x, y)$ and partial derivation of $G(x, y, \sigma)$ along $x, y$ :

$$
\left|\begin{array}{l}
F_{x}(x, y) \\
F_{y}(x, y)
\end{array}\right|=T(x, y) *\left|\begin{array}{l}
G_{x}(x, y, \sigma) \\
G_{y}(x, y, \sigma)
\end{array}\right|
$$

Where $F_{x}(x, y)$ and $F_{y}(x, y)$ are the derivatives of the image in the $x$ and $y$ directions.

One of the pixels $(x, y)$ 's gradient magnitude and direction in image is given by $F(x, y)$ and $\theta$,

$$
\begin{aligned}
& \text { Where } F(x, y)=\sqrt{F_{x}^{2}(x, y)+F_{y}^{2}(x, y)} \\
& \text { And } \quad \Theta=\arctan \frac{F_{y}(x, y)}{F_{x}(x, y)}
\end{aligned}
$$

Canny edge detection can detect all the edges which is present in the skin in silent region. 
4.2.4.2, SURF: We use SURF (Speed Up Robust Feature) [26] as a feature detector to extract the facial marks. We also applied Hessian matrix base detector because of its good performance in computation time and accuracy. Therefore, we apply Hessian-Laplace detector [76] rather than using a different measure for selecting the location and the scale and we rely on the determinant of the Hessian for both. The Hessian matrix $\mathrm{H}(\mathbf{x}, \sigma)$ in $\mathbf{x}$ at scale $\sigma$ is defined as follows:

$$
\mathcal{H}(\mathrm{x}, \sigma)=\left[\begin{array}{ll}
L_{x x}(\mathrm{x}, \sigma) & L_{x y}(\mathrm{x}, \sigma) \\
L_{x y}(\mathrm{x}, \sigma) & L_{y y}(\mathrm{x}, \sigma)
\end{array}\right]
$$

where $L_{x x}(\mathbf{x}, \sigma)$ is the convolution of the Gaussian second order derivative $\frac{\partial^{2}}{\partial x^{2}} \mathbf{g}(\sigma)$ with the image $I$ in point $\mathbf{x}$, and similarly for $L_{x y}(\mathbf{x}, \sigma)$ and $L_{y y}(\mathbf{x}, \sigma)$. To complete the procedure of facial mark detection process is enumerated below:
a) Landmark detection from the face using AAM.
b) Mapping to mean shape $S_{\mu}$.
c) Mask construction.
d) Apply Canny edge detector to detect the edges.
e) SURF is applied to extract the features.
f) Represent the marks with bounding box.

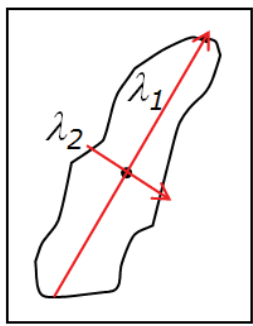

(1) Linear

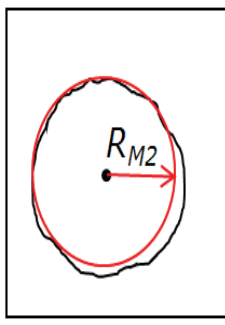

(2) Circular 


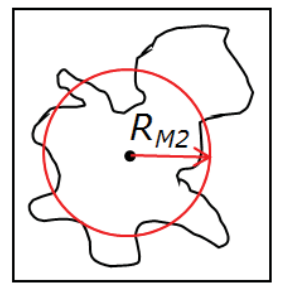

(3) Irregular

Figure 4.7, Mark classification using morphology.

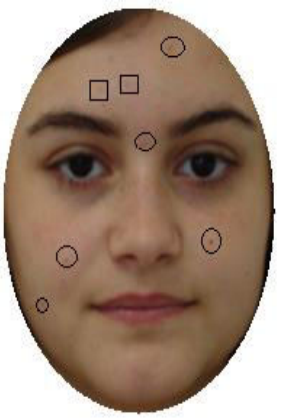

(1)

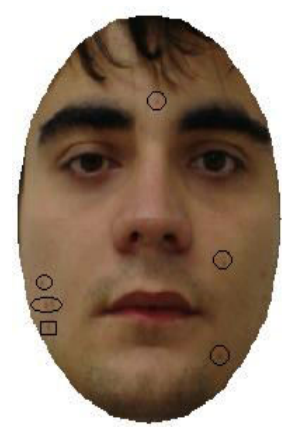

(2)

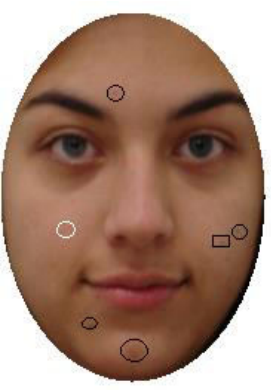

(4) 


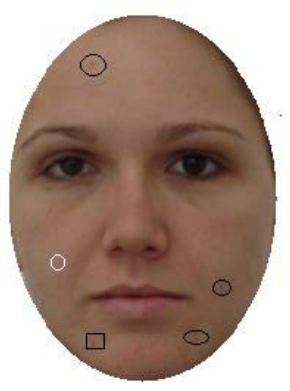

(5)

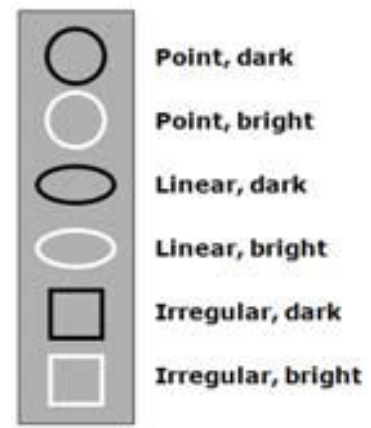

(6)

Figure 4.8, Examples of mark detection and classification results. (1),(2), (3), (4), (5) and (6) Symbols are used to denote six different classes of marks.

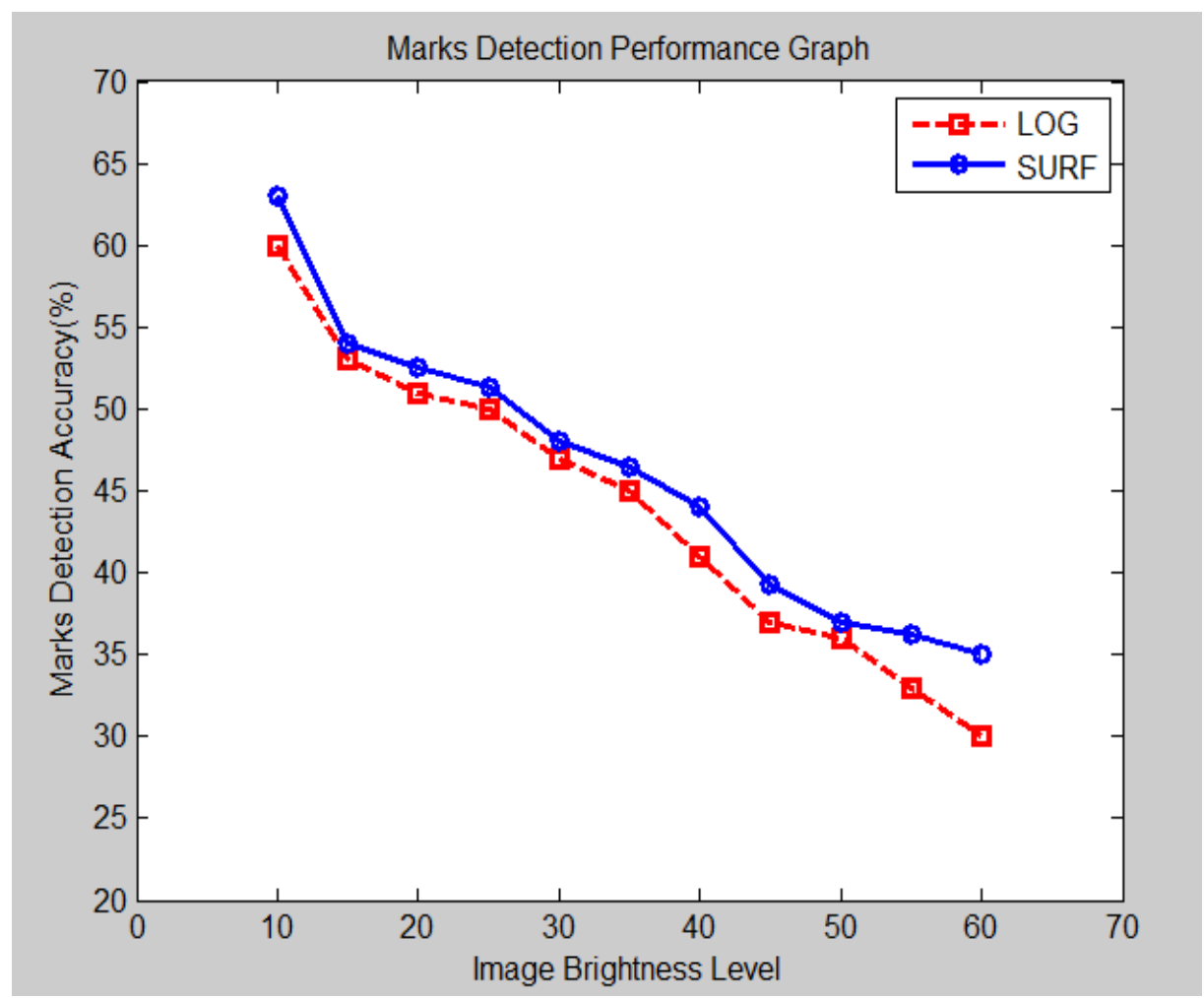

Figure 4.9, Mark detection graph

LOG is the existing mark detection performance.

SURF is the our proposed mark detection performance. 
Mark detection is evaluated in terms of mark detection accuracy and image brightness, shown in figure 4.9. The mark detection accuracy of mark detection with the range of brightness contrast varies from $(30 \%, 60 \%)$ to $(60 \%, 10 \%)$ in existing work and our proposed work varies from ( $35 \%, 64 \%)$ to ( $60 \%, 10 \%)$ from the face image. These results showed the automatic mark detection accuracy and performance. Our mark detection performance graph Fig. 4.9, shows that marks detection accuracy is better than the existing marks detection accuracy. We evaluated the automatic mark detection process which helps us to improve the face recognition accuracy.

\subsubsection{Facial Marks Blob Classification}

Each detected blob such as Marks, Moles etc. are assigned a bounding box tightly enclosing the blob. Then we classify a mark in a hierarchical fashion: linear versus all, followed by circular point versus irregular. Linearity classification of a blob used two eigenvalues such as $\lambda_{1}$ and $\lambda_{2}$ are obtained from the eigen decomposition on $x$ and $y$ coordinates of blob pixels. When $\lambda_{1}$ is larger than $\lambda_{2}$, the mark is considered as a linear blob. We calculate the second moment of the blob pixels $M_{2}$ for the circularity detection. A circle $R_{2} M_{2}$ with radius $M_{2}$ will enclose most of the blob pixels if they are circularly distributed. Therefore, a decision can be made based on the ratio of the number of pixels within and outside of $\mathrm{RM}_{2}$. The color of the blob can be decided based on the ratio of the mean intensity of the pixels inside and outside of the blob $\left(\mathrm{e}_{\text {in }} / \mathrm{e}_{\text {out }}\right)$.

The classification of blobs is shown in the figures 4.7 and 4.8 shows example images of facial marks detection results with ground truth and automatically extracted facial marks using our new method which is covered by cosmetic items. We demonstrated that our proposed method detects the facial marks robustly detected. 


\subsubsection{FACIAL MARK BASED MATCHING}

We encoded the detected facial marks into a 48 bins histogram representing the morphology, color, and location of facial marks. To encode the facial marks, the face image is subdivided into eight different regions in the mean shape space given in Figure 4. Each mark is encoded by six digit binary number representing its morphology and color. If facial marks are more than one found in the same region, a bit by bit summation is applied. The six bin values are concatenated for the eight different regions in the order as shown in Figure 4.9.1, to generate the 48 bin histogram. If a mark is obtained on the borderline of the face segments, it is considered in both regions also considering the variations of the segments across multiple face images of the same subject. The soft biometric traits based matcher is used to retrieve the data from the database. Soft biometrics can be combined with any facial matcher to improve the overall accuracy [22]. The weights are chosen to obtain the best face recognition accuracy.

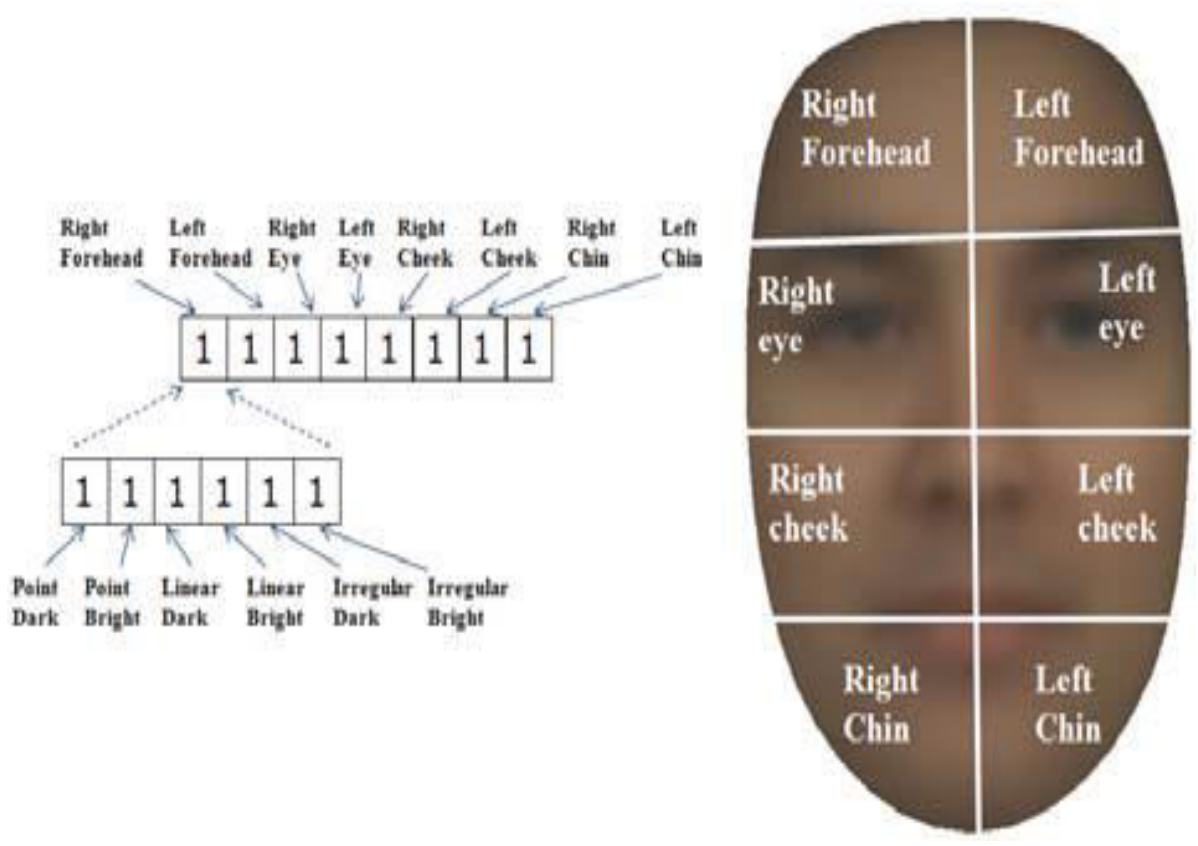

Figure 4.9.1, Schematic of the mark based indexing scheme. 


\subsection{EXPERIMENTAL RESULTS}

\subsubsection{Database}

We used two different kinds of databases, CVL database [83] and CMU PIE database [84][85] to design two separate databases, DB1 and DB2 are used to evaluate the proposed mark-based matcher. DB1 consists of images from CVL and CMU PIE databases with 1000 images, one image per subject, for probe and 2000 images, one per subject for the gallery. DB2 consists of CMU PIE face images with 1000 images from 1000 subjects for probe and 2000 images from 2000 subjects for the gallery. The image size varies from $384 \times 480$ to $640 \times 480$ (width height) for the CMU PIE and $1000 \times 2000$ for CVL databases both at 96 dpi resolution. Therefore, we manually labeled the fifteen facial mark types for DB2 and eight facial mark types (e.g., based on color and morphology) for the CVL images in DB1 to create the ground truth. This allows us to evaluate the proposed facial mark extraction method. We used the images from the CVL database in DB1.

\subsubsection{Face image matching and retrieval}

We measure the automatic mark detection method in terms of recall and precision value. Recall and precision are commonly utilized as performance evaluation in statistical classification and information retrieval tasks [86]. These measures are defined in terms of false positive, true positive and false negative. False positives are the number of automatically detected marks that are not in the ground truth, true positives are the number of automatically detected marks that match the ground truth, and false negatives are the number of ground truth marks that were not detected by the automatic detector.

Facial marks play a critical role to recognize the given face. We focused on detecting the facial marks which are permanently on the face. Therefore 
some of the facial marks are not stabilizing in human face for example pimples, acne or zits. To enhance the matching accuracy, we fixed up the permanent marks such as moles, scar, birth marks etc. on the face image. It will improve performance of the recognition or identification of the human face. Also we focused those face images that includes facial marks but that are covered by cosmetic items. Our method detects the all kind hidden facial marks on the face image. We simply filter out the unnecessary facial edges and fix the particular importance of facial marks. It improves the matching accuracy and enhanced the results. We collected a small amount of facial images which contains 1000 images. We retrieved the images and it shows $93 \%$ of accuracy from the face database. Figure 4.9.2, shows the detected facial marks that are retrieved from the database. Our database contains numerous detected face images. We have two databases DB1 and DB2; DB1 data base contains the facial marks detected images and DB2 database contains the face matching data. Our proposed mark detection method is implemented using Matlab 7.10.0(R2010a).

Table 4.9.2, Proposed facial marks matcher \& face recognition accuracy

\begin{tabular}{|l|c|c|}
\hline $\begin{array}{l}\text { Automatic marks detection } \\
\text { accuracy }\end{array}$ & Existing method & $\begin{array}{l}\text { Proposed method } \\
\text { ( Cosmetic applied } \\
\text { face) }\end{array}$ \\
\hline Based on facial marks & $92.10 \%$ & $93 \%$ \\
\hline
\end{tabular}
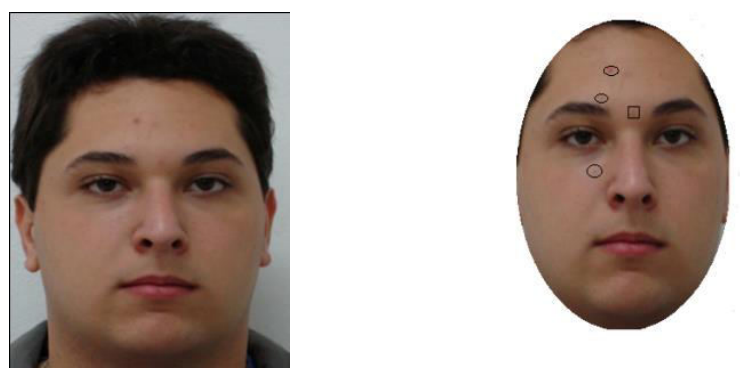

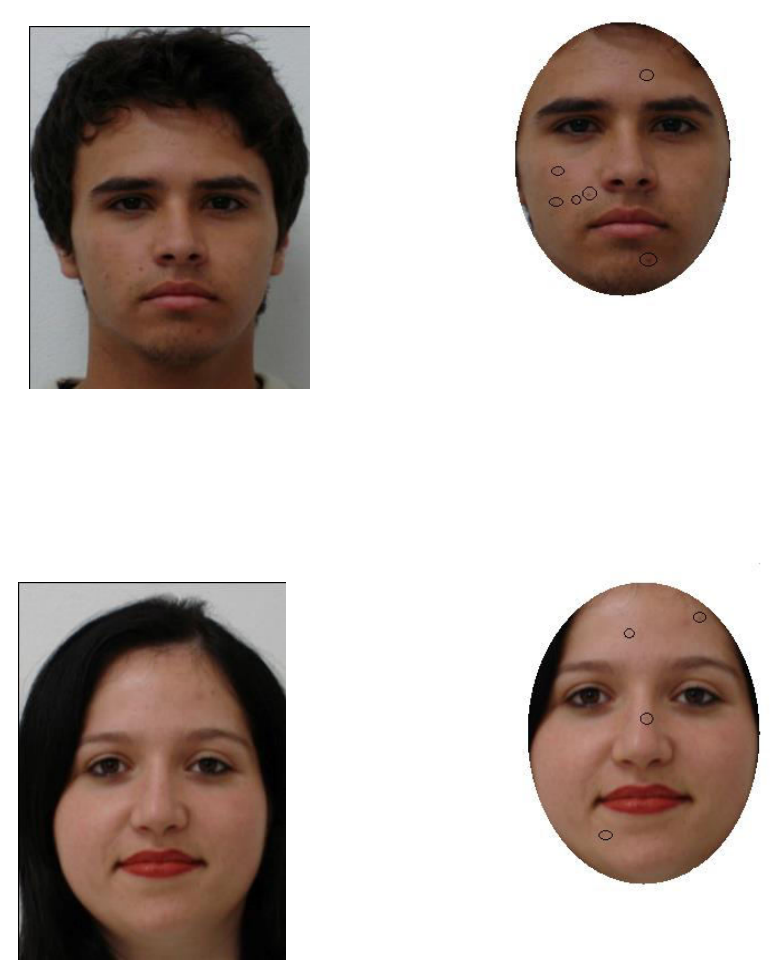

Figure 4.9.2. Examples of face image retrieval result.

\subsection{SUMMARY}

Facial marks from the cosmetic applied faces such as e.g., freckles, moles, and scars are salient localized regions appearing on the face which is to be useful in face recognition and provide more security in biometric authentication. An automatic facial mark extraction methodology has been developed that shows good performance in terms of matching and retrieval. The fusion of facial marks with a state of the art face matcher improves the face recognition performance. This shows that micro-level features such as facial marks do offer some discriminating information to enhance the security. Most of the facial marks detected are semantically meaningful so the users can issue queries to retrieve images of interest from a large database. 


\section{BIOMETRICS SECURITY: FACIAL MARKS DETECTION FROM THE LOW QUALITY IMAGES}

\subsection{INTRODUCTION}

A number of studies were examined to improve the face recognition by developing features representation schemes. These features include the salient skin regions, which appear on the human face such as scars, moles, freckles, wrinkles, etc. [10]. Previous studies illustrate that facial marks are primarily focused upon in evaluating facial recognition performance using standard face image data set. Park and Jain [73] expressed that facial marks are very essential in identifying twins using the semi automatic concept. They also labelled the demographic information such as ethnicity and gender. To detect the facial features the authors applied AAM manually and facial marks are detected using LoG with morphological operator. This method though was not enough to detect the facial marks from the low quality images [73]. But, facial marks have been used to speed up the retrieval process in order to differentiate the human faces [77].

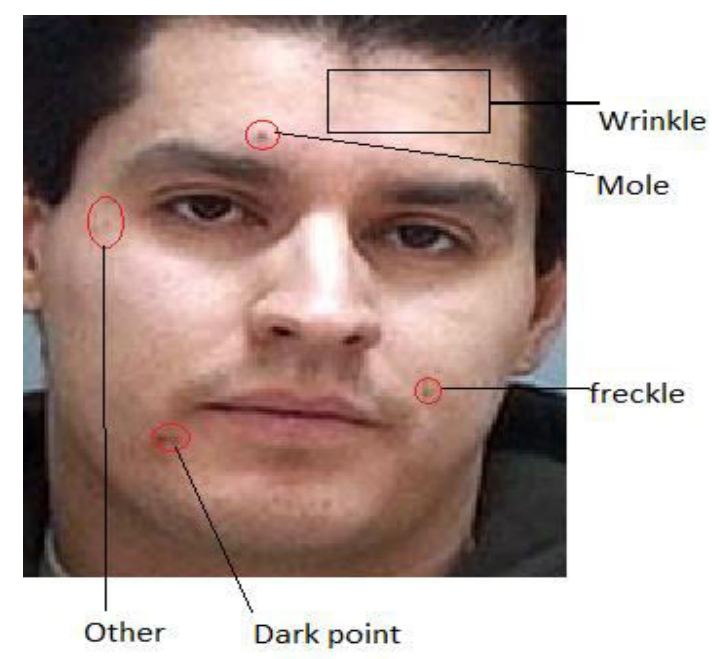

Figure 5.1, Example of low quality image with different facial marks. 
Figure 5.1, shows the example of different kinds of facial marks on the human face. An existing study found that for the face recognition purpose facial marks have been utilized very rarely [73][70]. Also Spaun [9][10] explained that facial examination has to provide identification of "class" and "individual" characteristics. The 'class' involves the overall facial shape, presence of hair, hair color, shape of nose, presence of marks etc. Similarly 'individual' characteristics involve the number \& location of scars, tattoos, location wrinkles etc. on a face. Lin at el. [6] first utilized the SIFT operator [16] to extract facial irregularities and fused them with global face matcher. However, the individual types of facial marks are not defined. Therefore their method is not suitable for face database indexing. Krystian Mikolajczyk and Cordelia Schmid et al [78] introduced GLOH is a descriptor which extends SIFT by changing the location grid and using PCA to reduce the size. It is also designed to increase its robustness and distinctiveness. Lee et al. [2] introduced "scars, marks, and tattoos (SMT)" in their tattoo based image retrieval system. While tattoos can be drawn or made at any point of the body, the study of face recognition with tattoos assumes greater significance. Pierrard et al. [70] proposed a method to extract moles using normalized cross correlation method and a morphable model. They claimed that their method is pose and lighting invariant since it uses a 3D morphable model. They did not consider other types of facial marks besides moles. Eigen faces [79] was one of the first successful face recognition methods. It can handle variations and blur in the images quite well, but it requires a large training set of images. Figure 5.2, shows how marks based matcher helps in indexing each face image based on facial marks. These indices enable fast retrieval and also for textual or key word base query. 


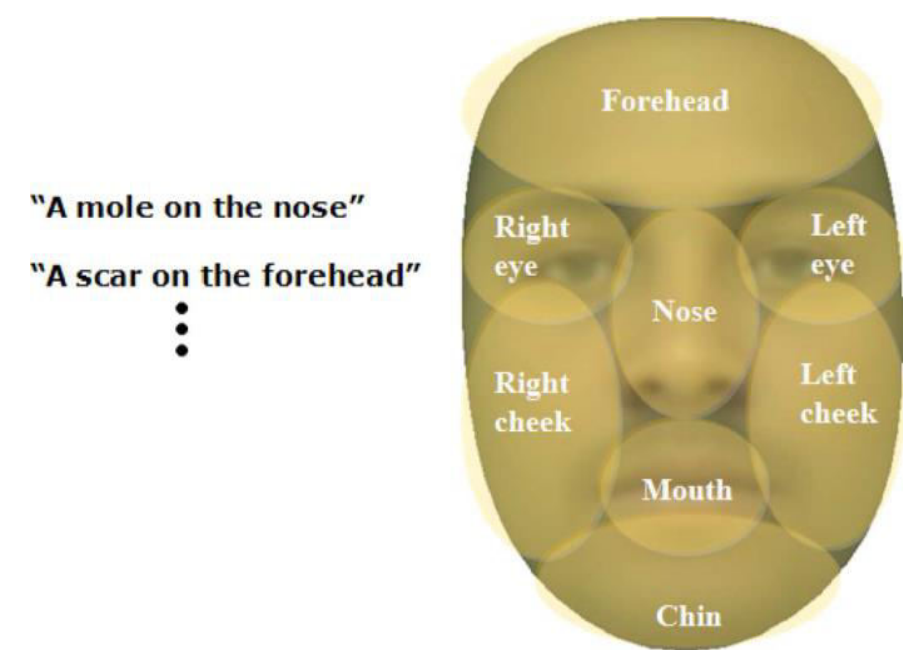

Figure 5.2, Example of textual query and a schematic of face region segmentation.

\subsection{PROPOSED METHOD}

Park and Jain et al.[73] used AAM (Active Appearance Model) using PCA (Principle Component Analysis) to detect the facial landmark from the face image. The local irregularities that are facial marks (moles, scars etc.) are extracted using LoG or DoG and morphological operators. Their methods are applicable only for normal with high quality images. In the literature we found that their methods are poor to detect the facial marks from the low quality images. In view of this we considered the low quality images due to sparse dark or light disturbances known as salt and pepper noise type. Generally these types of marks affect only a small number of image pixels. Typical sources of noise include flecks of dust inside the camera as well as from faulty CCD (Charge Coupled Device) elements. To overcome these inherent problems, we have proposed a facial mark detection method that can be applied on low quality images. Our proposed method differs significantly from the existing study in determining the facial marks from the low quality images. Figure 5.4, shows 
images which contain salt and pepper noise, (i.e. sparse dark or light disturbances). Initially, we filter out those face images containing salt and pepper noise using the Adaptive Median Filter (AMF). Therefore to detect the landmarks from the face image, we applied powerful techniques ASM (Active Shape Model) into AAM (Active Appearance Model) using PCA (Principle Component Analysis). We also subtract the unwanted facial features such as eyebrows, eyes, nose, and mouth from the face image. Finally, the local irregularities such as scars, moles, freckles etc. are extracted using GLOH (Gradient Location Orientation Histogram). Our proposed method showed the best result contrast with existing study. The complete architecture of the proposed method with necessary steps involved in our research work is given in fig 5.3. Our proposed method differs from the previous works in the following aspects; (i) we concentrate on filtering the face images which contain salt and pepper type. (ii) All the facial marks that are locally salient due to low quality are extracted. (iii) We concentrate on finding semantically meaningful facial marks instead of extracting texture patterns that implicitly include facial marks. These proposed facial marks determination concepts can be helpful in forensics and law enforcement agencies as it can supplement existing facial matchers to improve the identification accuracy.

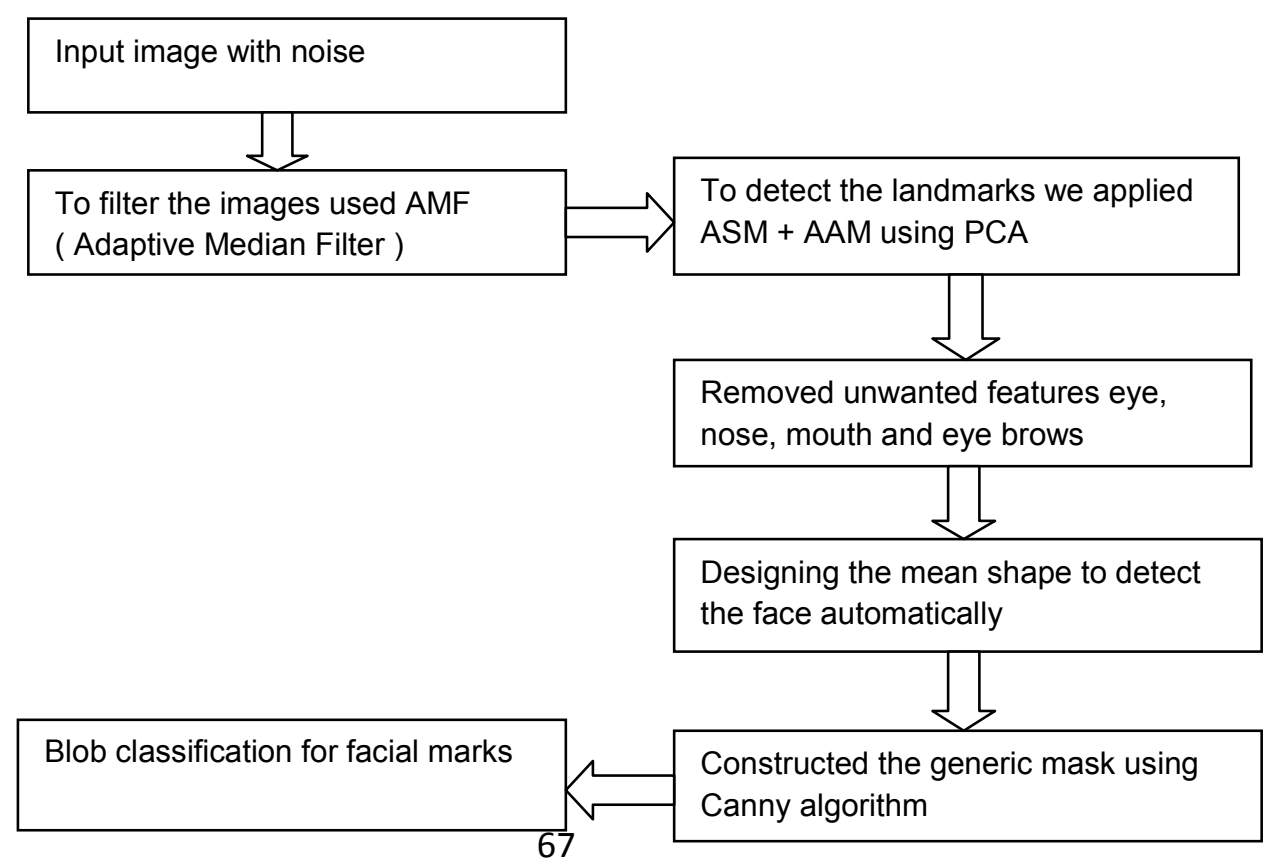




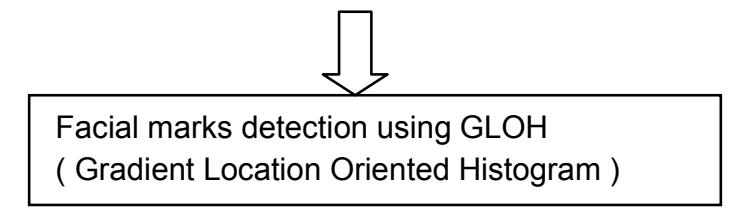

Figure 5.3, Overall structure of our proposed method.

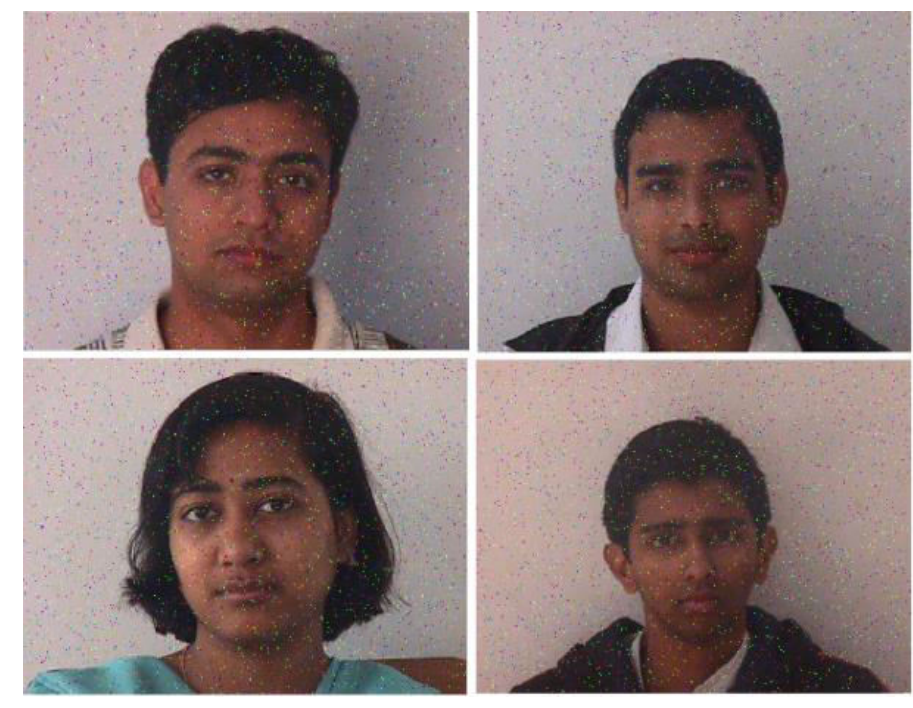

Figure 5.4, shows salt and pepper noise images

\subsection{FACIAL MARKS ON THE FACE}

Facial marks are located in different regions of the face. To know the different categories of facial marks present in face, we need to analyse the categories of marks. Different kinds of facial marks are freckle, mole, scar, pockmark, acne, whitening, dark skin etc. Freckle is a single or a set of dark spots present in the face. Wherever there was a dense set of spots we labelled them in a single bounding box. A mole typically appears large in size and darker in color compared to the other spots. Scar represents the discolored region in the skin 
due to a cut or injury. Acne is a red region caused due to a pimple and is stable for a few days to several months. Whitening represents a skin region that appears brighter in contrast with the surrounding region. We took into consideration the wrinkles that are larger and omitted the smaller wrinkles near the eyes and mouth. We also ignored the beard and facial hair in constructing the ground truth. All other kinds of marks which are not mentioned above are labelled under the "others" group. Figure 5.5, shows the different facial marks in a facial image. The average number of marks observed in our database is 9 per subject. All the images in the database showed at least 2 marks per subject.

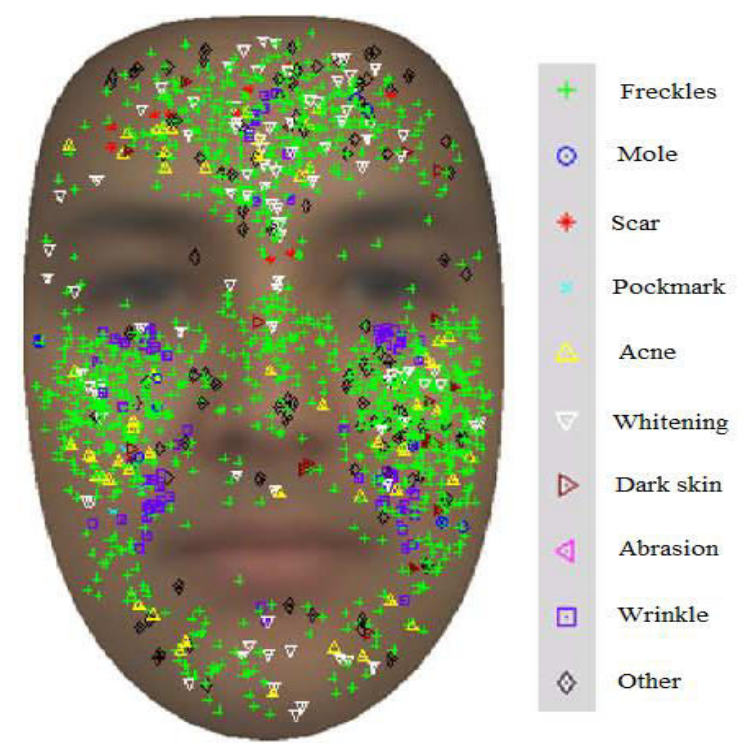

Figure 5.5, Different facial marks on the mean face image

\subsection{FACIAL MARKS DETECTION METHOD}

The proposed mark detection method is based on the Gradient Location Orientation Histogram (GLOH) [78] that detects local silent points of a low quality input image or a noisy image. Therefore, to detect the facial feature, it will increase the local extrema of facial features such as eyes, eyebrows, nose, 
and mouth. To avoid detecting unwanted local facial features we subtracted eyes, eyebrows, nose, and mouth from the face image using ASM into AAM using PCA. To detect the facial landmark we applied ASM into AAM using PCA and we provide masking process using [21]. The complete facial mark detection process is illustrated in Figure 5.6, with the following steps 4.1) Noise removal 4.2) Facial feature detection 4.3) Designing the mean shape and mask construction 4.4) Gradient Location Orientation Histogram and 4.5) Blob classification for facial marks.
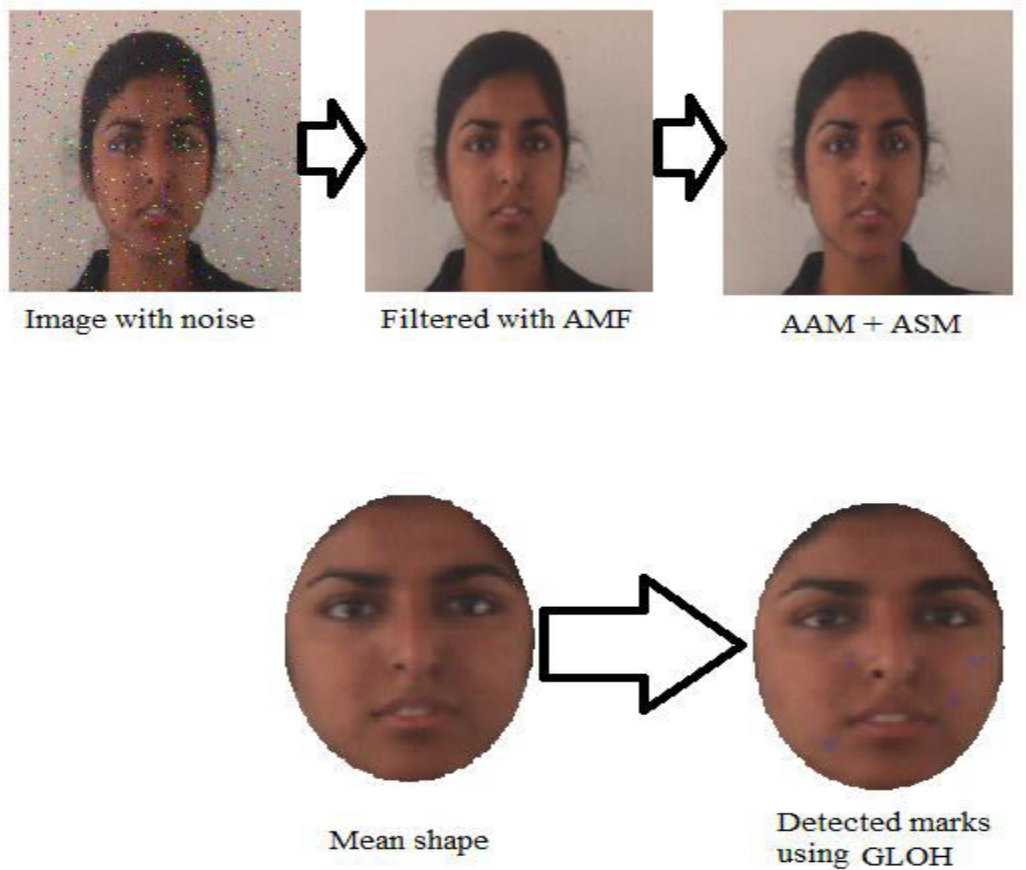

Figure 5.6, Schematic of Automatic Facial Mark Extraction Process.

\subsubsection{Noise Removal}

Adaptive Median Filter (AMF) [80] is applied to remove the noises (salt and pepper noise) from the face image and performs spatial processing to find out 
the pixels that have been affected by impulse noise in an image. Moreover, it groups pixels as noise by comparing each pixel in the image in its neighbouring pixels. A pixel which is different from a majority of its neighbours, as well as being not structurally aligned with those pixels to which it is similar, is defined as impulse noise. The median pixel value of the pixels in the neighbourhood that have passed the noise labelling test then replace these noise pixels. The filtered image from salt and pepper noise type using AMF is shown in Figure 5.7, and Figure 5.8, 5.9, shows the histogram of an image. The purpose of applying the AMF is

1). Removing impulse noise from the image.

2). Smoothing of other noises from the image.

3). Reducing distortion, like excessive thinning or thickening of object boundaries.

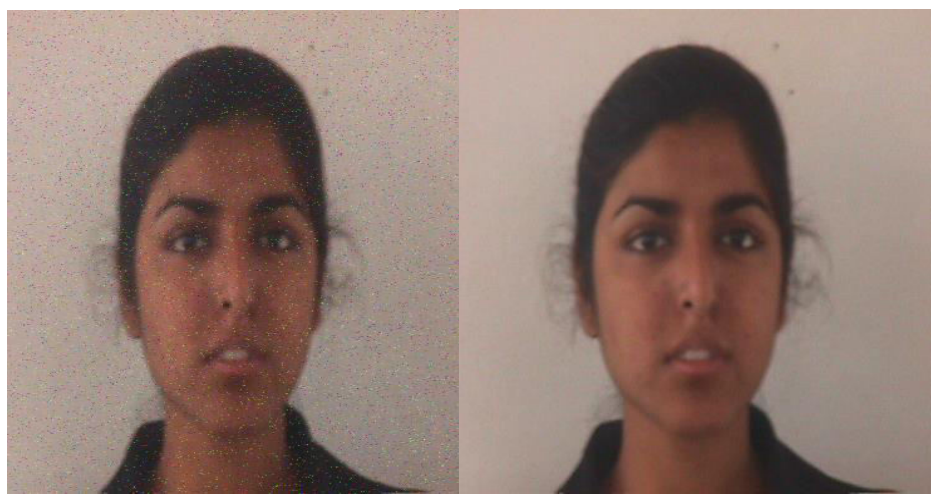

Noise with salt \& pepper Filtered with AMF

Figure 5.7, Salt and pepper noise filtered with AMF 


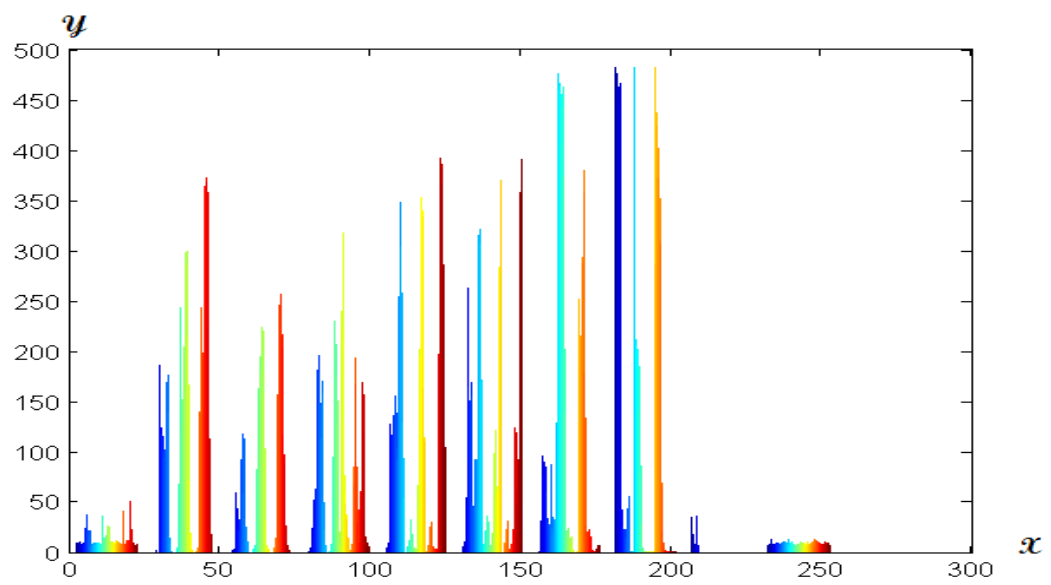

Figure 5.8, shows histogram for noisy color image

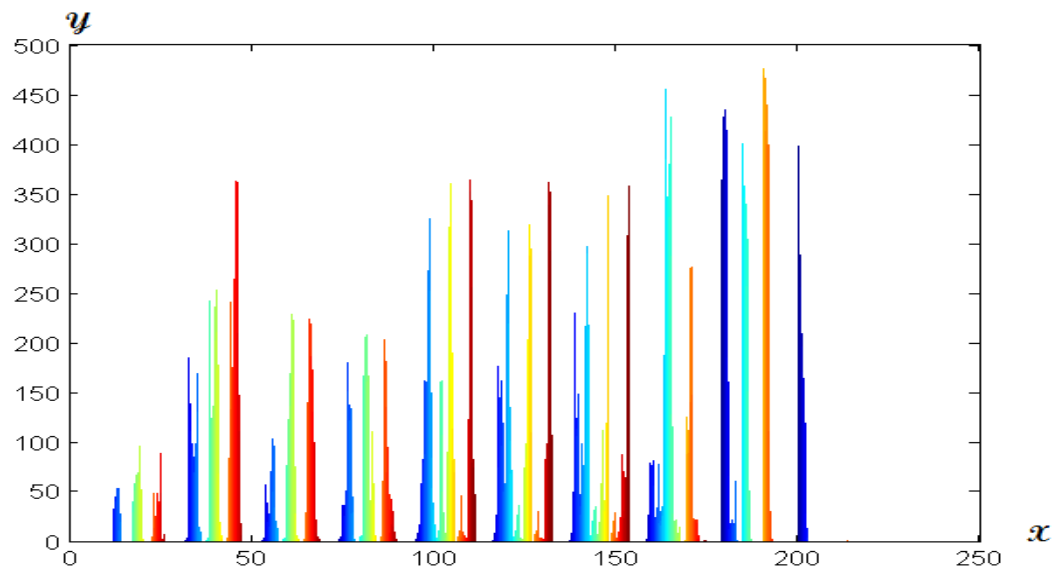

Figure 5.9, shows histogram for noiseless color image

A histogram of an image is produced first by discretization of the colors in the image into a number of bins, and counting the number of image pixels in each bin. The $x$ axis of the graph represents the tonal variation, while the $y$ axis represents the number of pixels in that particular tone. 


\subsubsection{Facial Feature Detection}

The integrated Active Shape Model (ASM) into Active Appearance Model (AAM) [21] is applied to detect automatically 80 landmarks that delineate the facial features such as the mouth, nose, eyes, eyebrows and face boundary. For reliability, we first detect the landmarks of two eyes, nose and mouth. These facial features will be disregarded in the subsequent facial mark detection process. ASM into AAM identifies both the shape and texture of face images using the Principle Component Analysis (PCA). ASM finds the shape parameters so that the profile of each model is similar to the pre-learned profile. It is another way of keeping the shape parameter within the learned range in the parameter space. Similarly, AAM finds the shape and appearance model parameters such that the model instance is most similar to the input image. Models that are obtained from the current model parameter are as similar as the input image. ASM and AAM are hence combined to reduce the error rate and detect the face with perfect landmark points.

\subsubsection{Designing Mean Shape and mask construction}

(5.4.3.1) Active Shape Model in Active Appearance Model has been applied to detect the landmarks, thereby to simplify the mark detection. We then map each face image to the mean shape. Consider that $S_{i}$ where $i=1,2 \ldots, N$ represents the shape of each of the $\mathrm{N}$ face images in the database (gallery) based on the 80 landmarks. The mean shape is calculated using the equation $S_{\mu}=\sum_{i=1}^{N} S i$.

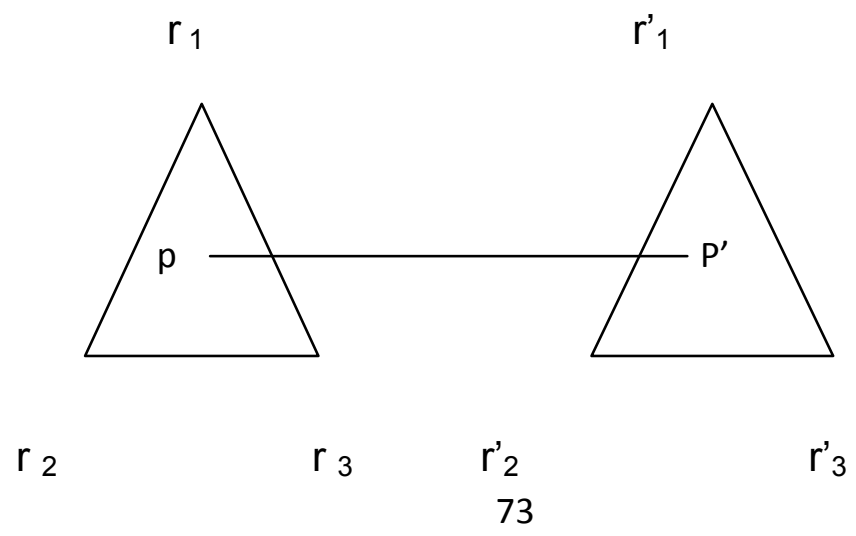


Figure 5.9.1, Schematic of the texture mapping process using the triangular Barycentric coordinate system.

The schematic of a mean face construction system is shown in Figure 5.9.1. Each face image $S_{i}$ is mapped to the mean shape $S_{\mu}$ by using the Barycentric coordinate-based [75] texture mapping process. Initially, both $S_{i}$ and $S_{\mu}$ are subdivided into a set of triangles in such a way that given a triangle $T$ in $S_{i}$, its corresponding triangle $T^{\prime}$ is found in $S_{\mu}$. Let $r_{1}, r_{2}$, and $r_{3}\left(r_{1}^{\prime}, r_{2}^{\prime}\right.$ and $\left.r_{3}^{\prime}\right)$ be the three vertices of $T\left(T^{\prime}\right)$. Then, any point $p$ inside $T$ is expressed as $p=\alpha r_{1}+\beta r_{2}+$ $\gamma r_{3}$, and the corresponding point $p^{\prime}$ in $T^{\prime}$ is similarly expressed as $p^{\prime}=\alpha r^{\prime}{ }_{1}+\beta r^{\prime}{ }_{2}+$ $\mathrm{yr}_{3}{ }_{3}$, where $\alpha+\beta+\gamma=1$. In this way, the pixel value is mapped and shown in Figure 5.9.2, using the schematic of the Barycentric mapping process.

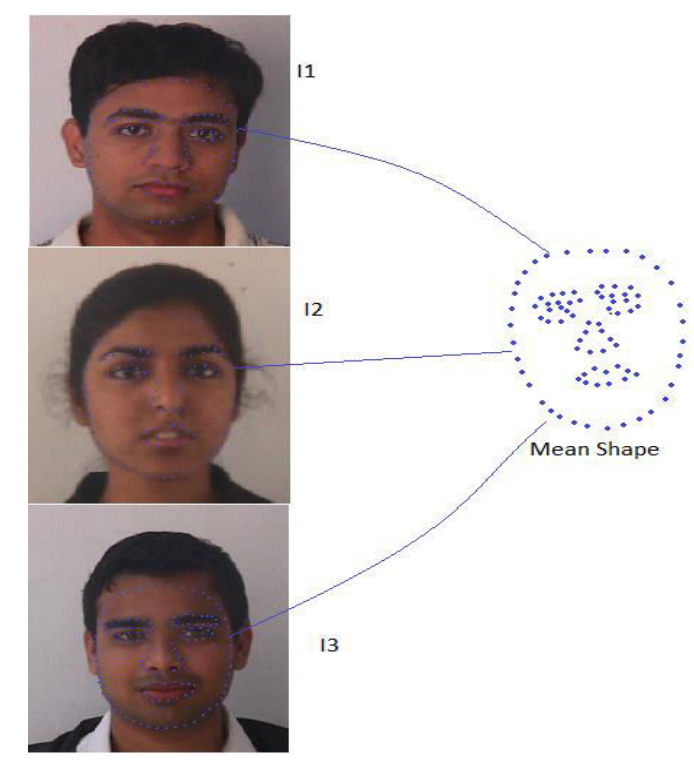

Figure 5.9.2, Schematic of the mean face construction.

This mapping process is repeated for all the points which are present inside the triangle and the texture in $S_{i}$ is mapped to $S_{\mu}$. Hence, after the mapping process, all face images are normalized in terms of scale and rotation and hence this facilitates us to represent each facial mark in a face-centered common 
coordinate system. Fig. 5.9.2, shows the schematic of mean face construction. (5.4.3.2) We construct a generic mask and derive a user specific mask to suppress false positive value detection around the facial feature. The user specific mask covers small unusual landmarks around the facial feature. We suppress the false positives of small wrinkles or beard that are connected to the facial feature. We then build a user specific mask from the edge image obtained using the canny edge detector [23].

\subsubsection{Gradient location and orientation histogram (GLOH)}

Gradient location and orientation histogram (GLOH) [78] is a descriptor which extends SIFT by changing the location grid and using PCA to reduce the size. It is also designed to increase its robustness and distinctiveness. The SIFT descriptor is computed for a log-polar location grid with 3 bins in radial direction i.e. the radius set to 6,11 and 15 and 8 in angular direction, which results 17 location bins. It is to be mentioned that the central bin is not divided in angular directions and the gradient orientations are quantized in 16 bins. It provides a 272 bin histogram and the size of this descriptor is reduced using PCA. The 128 largest eigenvectors are used for description. Shape context is similar to the SIFT descriptor, but it is based on edges. Shape context is a 3D histogram of edge point locations and orientations. Edges are extracted by using the Canny [22][23] detector. Location is quantized into 9 bins of a log-polar coordinate system. The radius set to 6,11 and 15 and orientation quantized into 4 bins (horizontal, vertical and two diagonals). This leads to a 36 dimensional descriptor which is allowed to detect the features present on the face image and it has shown to give better results than using the same weight for all edge points, as proposed in [81]. Therefore the original shape context was computed only for edge point locations and not for orientations. 


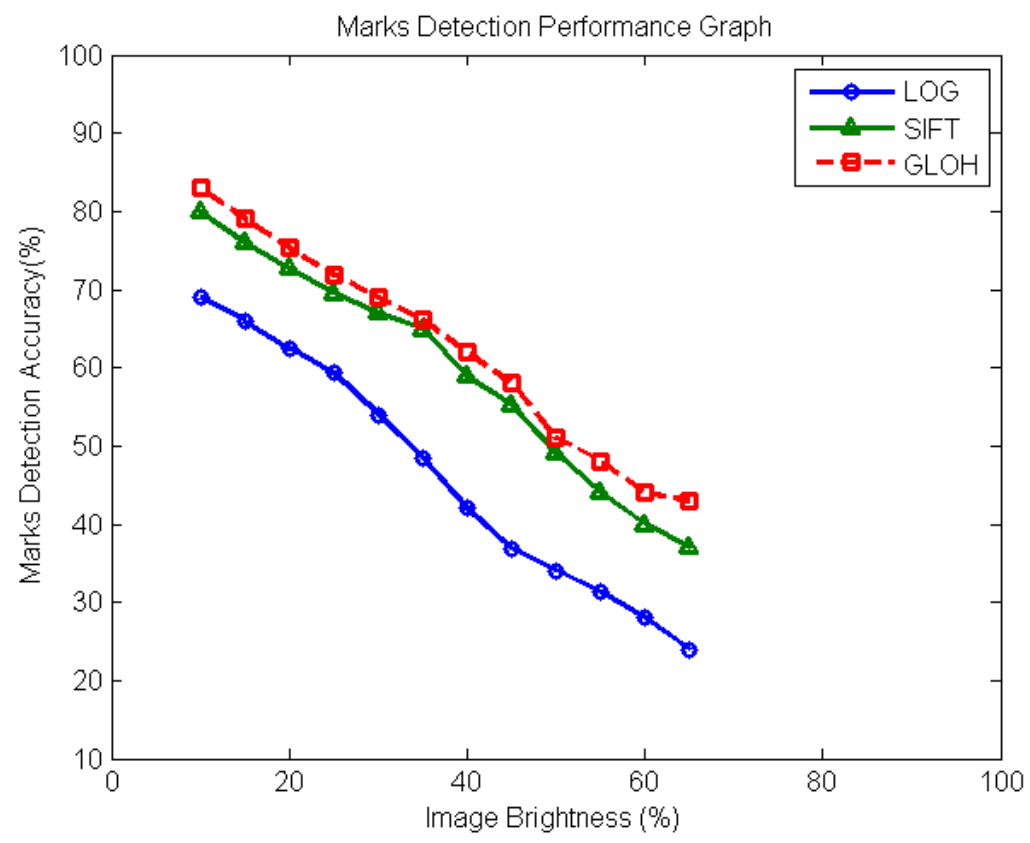

Figure 5.9.3, Marks detection performance graph

LOG, SIFT -- the existing mark detection performance.

GLOH -- the proposed mark detection performance.

\begin{tabular}{|l|l|l|l|l|l|l|l|l|l|l|l|l|}
\hline $\begin{array}{l}\text { Image } \\
\text { Brightness } \\
(\%)\end{array}$ & 10 & 15 & 20 & 25 & 30 & 35 & 40 & 45 & 50 & 55 & 60 & 65 \\
\hline LOG(MDA) & 69 & 66 & 62.5 & 59.3 & 54 & 48.5 & 42 & 36.9 & 34 & 31.3 & 28 & 24 \\
\hline SIFT(MDA) & 80 & 76 & 72.7 & 69.5 & 67 & 64.9 & 59 & 55.3 & 49 & 44 & 40 & 37 \\
\hline GLOH(MDA) & 83 & 79 & 75.4 & 71.9 & 69 & 66.1 & 62 & 58.1 & 51 & 48 & 44 & 43 \\
\hline
\end{tabular}

Table 5.9.3.1, Mark Detection Accuracy (MDA) in percentage 
Mark detection performance is evaluated in terms of mark detection accuracy and image brightness, as shown in figure 5.9.3. The $Y$ axis denotes the mark detection accuracy in percentage and $X$ axis denotes the image brightness lavel in percentage. The mark detection accuracy of mark detection with the range of brightness contrast varies from ( $26 \%, 65 \%)$ to $(69 \%, 10 \%)$ and $(37 \%, 65 \%)$ to $(80 \%, 10 \%)$ in existing work and our proposed work varies from ( $43 \%, 65 \%)$ to $(83 \%, 10 \%)$ from the face image. The mark detection accuracy is provided in the above table 5.9.3.1. The mark detection performance graph in fig. 5.9.3, shows that the marks detection accuracy by our method is better than existing marks detection accuracy. This helps to improve the face recognition accuracy. The mark detection accuracy is based on an Indian face database [82].

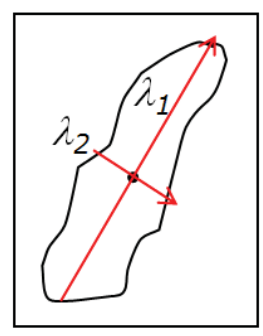

(1) Linear

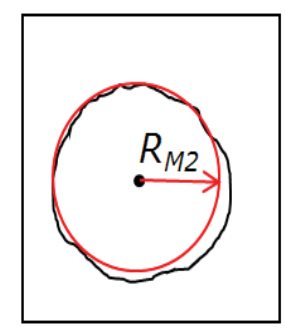

(2) Circular 


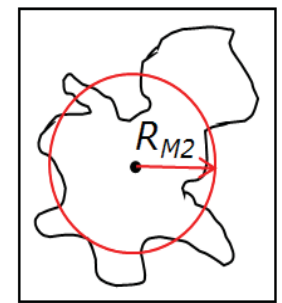

(3) Irregular

Figure 5.9.4, Mark classification using morphology.
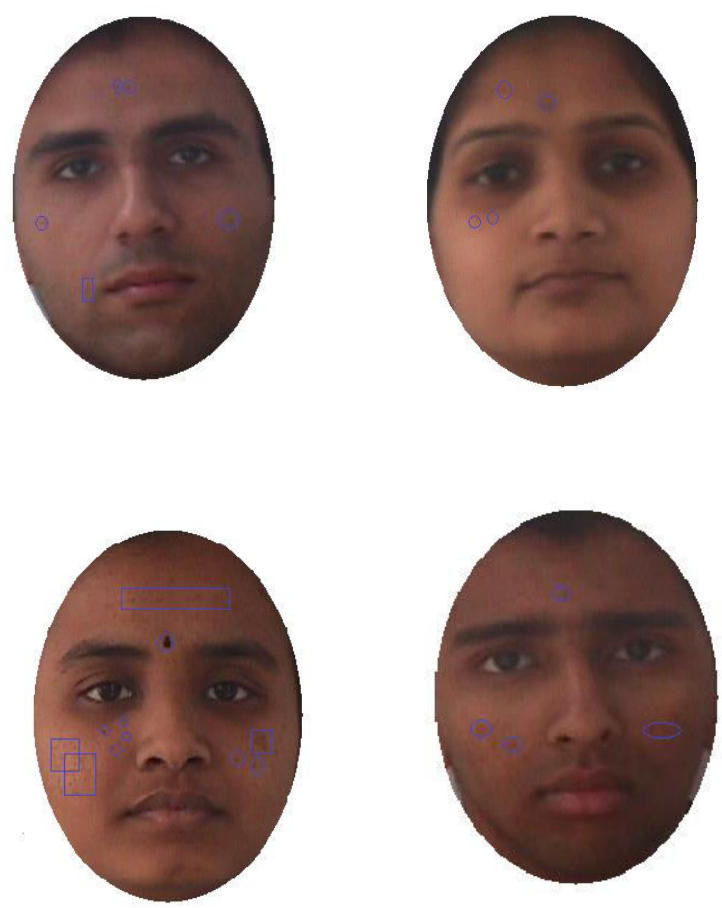

Figure 5.9.5, Examples of facial marks which are detected from face image.

\subsubsection{Blob Classification for Facial Marks}

The classification of blobs is shown in the figure 5.9.4. Each detected blob such as Marks, Moles, freckles etc. are assigned a bounding box. Pixels in the bounding box are binarized with a threshold value selected from the mean value 
of the surrounding pixels. Local extrema is brighter or darker than its surrounding region, so the average value of the surrounding area can serve effectively for fixing the bounding box. Then a mark is classified in a hierarchical fashion: linear versus all, followed by circular point versus irregular. In the linearity classification of a blob, $\lambda_{1}$ and $\lambda_{2}$ are the two eigenvalue that we obtained from the eigen decomposition on the $\mathrm{x}$ and $\mathrm{y}$ coordinates of blob pixels. When $\lambda_{1}$ is larger than $\lambda_{2}$, the mark is considered as a linear blob. Then the second moment of the blob pixels $M_{2}$ for the circularity detection is calculated. A circle $\mathrm{RM}_{2}$ with radius $\mathrm{M}_{2}$ will enclose most of the blob pixels. In view of this, a decision can be made based on the ratio of the number of pixels within and outside of $\mathrm{RM}_{2}$.

Figure 5.9.5, elaborates facial marks detection results using our new method.

\subsubsection{FACE BASED MATCHING FOR FACIAL MARKS}

We then encoded the detected facial marks into a 48 bins histogram representing the morphology, color, and location of facial marks. For encoding, the face image is subdivided into eight different regions in the mean shape space. Each mark is encoded by six digits binary numbers representing its morphology and color. If more than one facial mark is found in the same region, a bit by bit summation is applied. The six bin values are concatenated for the eight different regions in the order as shown in Figure 5.9.6, to generate the 48 bin histogram. If a mark is obtained on the borderline of the face segments, it is included in both regions considering the variation of the segments across multiple facial images of the same subject. Given the indices obtained from face images, the histogram intersection method is used to calculate the matching scores or filter the candidate face image. 

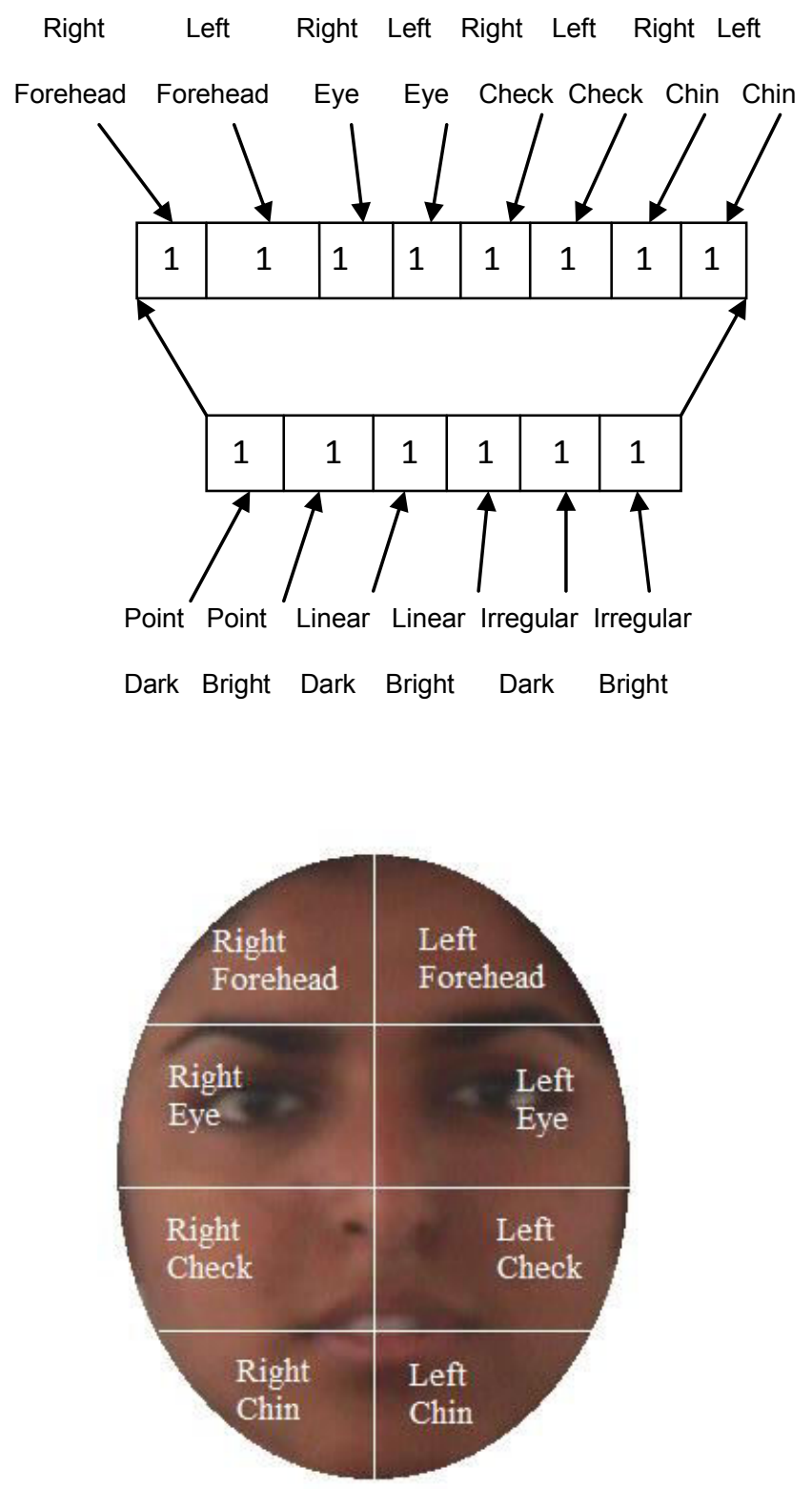

Figure 5.9.6, Schematic of the mark based indexing scheme. 


\subsection{EXPERIMENTAL RESULTS}

\subsubsection{Database}

The face images data sets which contain low quality images are named as DB1 and DB2 which are collected from an Indian face database [82]. DB1 and DB2 are used to evaluate the proposed mark-based matcher. We gathered 1000 images to demonstrate the proposed facial mark detection method. The image size in our database is $640^{*} 480$ (width * height) with 96 dpi resolution. We manually labeled different types of facial marks as defined in section III as ground truth. This process allows us to evaluate the proposed facial marks extraction method. DB1 is used to evaluate the effectiveness of the indexing scheme to improve the facial individuality. The soft biometric traits based matcher is used to retrieve the data from the database. It can be combined with any facial matcher to improve the overall accuracy [22]. Weights are chosen to obtain the best face recognition accuracy.

\subsubsection{Image matching and retrieval for security}

We measure the automatic mark detection method in terms of recall and precision value. Recall and precision are commonly utilized as performance evaluation in statistical classification and information retrieval tasks [86]. These measures are defined in terms of false positive, true positive and false negative. False positives are the number of automatically detected marks that are not in the ground truth, true positives are the number of automatically detected marks that match the ground truth, and false negatives are the number of ground truth marks that were not detected by the automatic detector.

We have used DB1 and DB2 for the soft biometrics retrieval and matching experiments. We applied our marks based matcher on facial images to identify the human face. The soft biometric matcher successfully retrieves the correct images to probe the face images. The facial feature points are manually 
labeled with 80 landmarks for the partial face and automatically labeled for the database images. We retrieved the images and it shows $95 \%$ of accuracy from the face database. Figure 5.9.7, shows the face matching and retrieval results in which all the marks are detected automatically from the database. As some of the facial marks are not stabilized in our face for example pimples, acne or zits, problems occurred during the matching. To enhance the matching accuracy we fixed up the permanent marks such as moles, scar, birth marks etc. on the face image. It improves the recognition or identification of the particular person. Our method detects majority of the facial marks from a low quality face image. We simply filtered out the unnecessary facial edges. It also improved the matching accuracy and enhances the results. The proposed mark detection method is implemented using Matlab 7.10.0(R2010a).
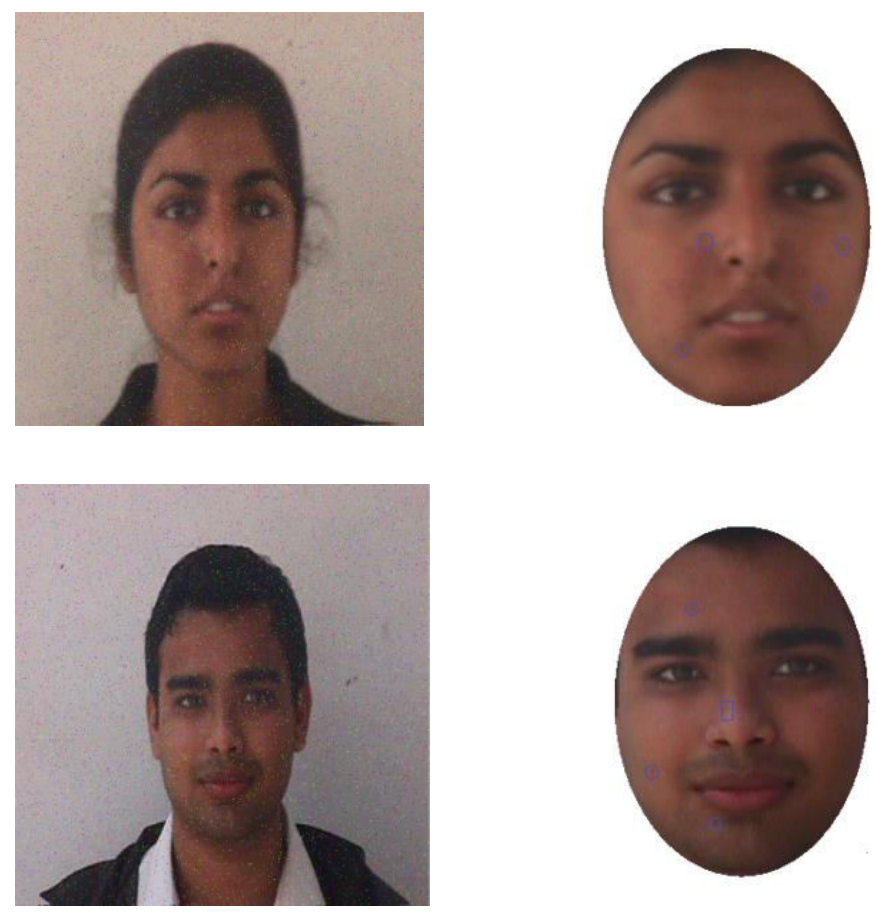

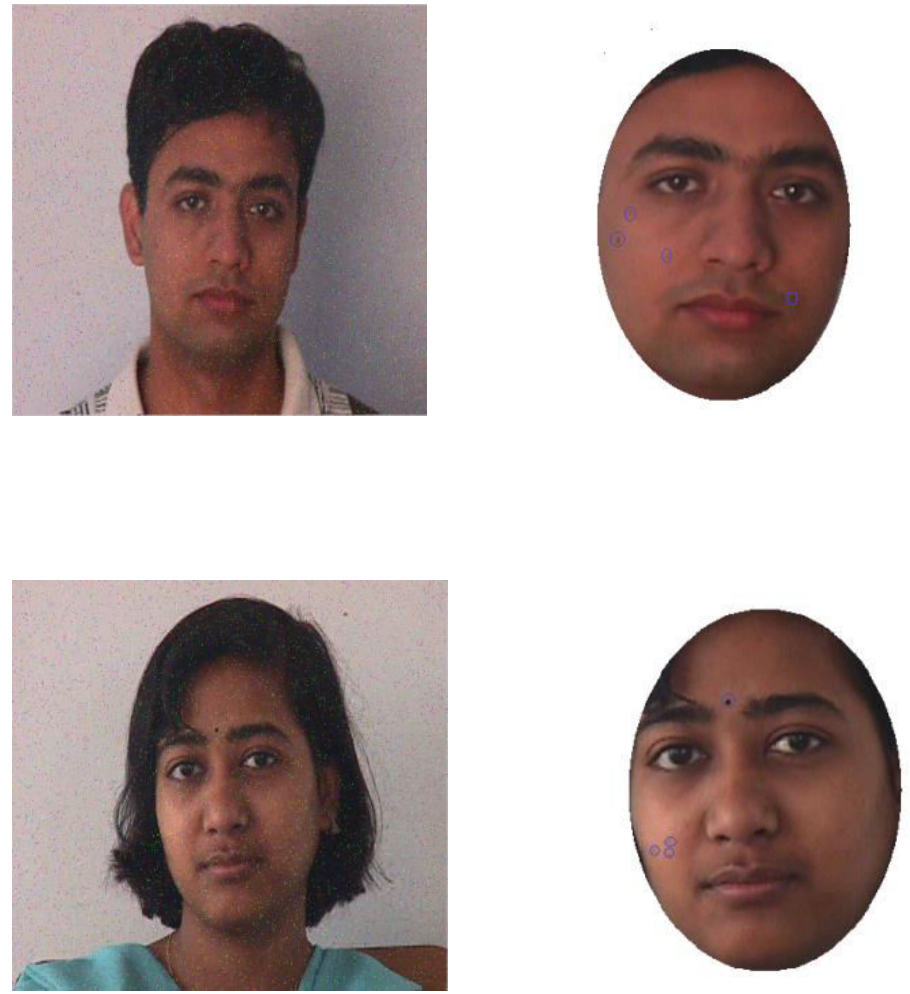

Figure 5.9.7, face matching and retrieval results

\subsection{SUMMARY}

Facial marks from the low quality images which is salt and pepper noise type due to sparse dark light disturbance due faulty CCD elements and extracting the features such as e.g., freckles, moles, and scars are salient localized regions appearing on the face which is to be useful in face recognition and provide more secure in biometric authentication. An automatic facial mark extraction methodology has been developed that shows good performance in terms of matching and retrieval. The fusion of facial marks with a state of the art face matcher improves the face recognition performance. This shows that micro-level features such as facial marks do offer some discriminating information to enhance the security. Most of the facial marks detected are semantically meaningful so the users can issue queries to retrieve images of interest from a large database. 


\section{CONCLUSION AND FUTURE DIRECTION}

\subsection{Conclusion}

We have proposed a new facial mark detection process as well as face matching which are covered by the cosmetic items on each face. We have shown that the proposed facial based indexing scheme helps us in matching the facial marks and to enhance the security based on biometrics. For marks detection and enhancing purpose, we used powerful canny edge detection method and SURF algorithm for feature extraction purpose. In contrast to the previous studies where the facial marks are presented implicitly and with poor accuracy, we presented the most effective method to detect the facial marks. We are currently working for further improvement with robust mark detection methods and we are also studying image resolution requirements for reliable mark extraction.

We have also proposed a new facial mark detection process from the low quality images. The images are filtered using the Adaptive Median Filter to improve the quality of images. We have shown that the proposed method is also useful for face matching and retrieval to enhance the security and in biometrics system. GLOH algorithm is used for feature extraction. In contrast to the previous studies that employed the facial marks implicitly or with poor accuracy, here we present the most effective method to detect the marks. We are currently working for further improvement in the different quality of face images to detect the facial marks. We are also studying image resolution requirements for reliable marks extraction. 


\subsection{Future Direction}

Based on the contributions of this thesis, the following research directions appear promising. Additional features such as morphology or color of the facial mark based matching should be considered. This will improve the matching accuracy with facial marks and enable more reliable face image retrieval. The face image retrieval system can be combined with other robust face matchers for faster search. Since each facial mark is locally defined, marks can be easily used in matching and retrieval given partial faces. The proposed facial mark detection, and matching system should be evaluated in an image based recognition systems. The mark based matching techniques can be combined to build a unified system for video based face recognition. 
Bibliography 


\section{REFERENCES}

[1] A. K. Jain and U. Park, "Facial marks: Soft biometric for face recognition," in Proc. IEEE ICIP, 2009, pp. 1-4.

[2] J.E. Lee, A. K. Jain, and R. Jin, "Scars, marks and tattoos (SMT): Soft biometric for suspect and victim identification," in Proc. Biometric Symposium, Biometric Consortium Conf., 2008, pp. 1-8.

[3] A. K. Jain, S. C. Dass, and K. Nandakumar, "Soft biometric traits for personal recognition systems," in Proc. ICBA, 2004, vol. 3072, LNCS, pp. 731-738.

[4] N. Kumar, A. C. Berg, P. N. Belhumeur, and S. K. Nayar, "Attribute and simile classifiers for face verification," in IEEE Int. Conf. Computer Vision (ICCV), 2009, pp. 1-8.

[5] J. S. Pierrard and T. Vetter, "Skin detail analysis for face recognition," in Proc. CVPR, 2007, pp. 1-8.

[6] D. Lin and X. Tang. "From macrocosm to microcosm." In Proc. CVPR, pages 1355-1362, 2006.

[7] S. Pamudurthy, E. Guan, K. Mueller, and M. Rafailovich, "Dynamic approach for face recognition using digital image skin correlation," in Proc. Audio- and Video-based Biometric Person Authentication (AVBPA), 2005, pp. 1010-1017.

[8] B. Klare and A. K. Jain, "Sketch to photo matching: A feature-based approach," in Proc. SPIE, Biometric Technology for Human Identification VII, 2010, pp. 1-10. 
[9] N. A. Spaun, "Facial comparisons by subject matter experts: Their role in biometrics and their training," in Proc. ICB, 2009, pp. 161-168.

[10] N. A. Spaun, "Forensic biometrics from images and video at the Federal Bureau of Investigation," in Proc. BTAS, 2007, pp. 1-3.

[11] L. Wiskott, J.-M. Fellous, N. Kruger, and C. von der Malsburg, "Face recognition by elastic bunch graph matching," IEEE Trans. Pattern Anal. Mach. Intell., vol. 19, no. 7, pp. 775-779, Jul. 1997.

[12] P. N. Belhumeur, J. P. Hespanha, and D. J. Kriegman, "Eigenfaces vs. fisherfaces: Recognition using class specific linear projection," IEEE Trans. Pattern Anal. Mach. Intell., vol. 19, no. 7, pp. 711-720, Jul. 1997.

[13] X. He, S. Yan, Y. Hu, P. Niyogi, and H.-J. Zhang, "Face recognition using laplacianfaces," IEEE Trans. Pattern Anal. Mach. Intell., vol. 27, no. 3, pp. 328-340, Mar. 2005.

[14] P. S. Penev and J. J. Atick, "Local feature analysis: A general statistical theory for object representation," Comput. Neural Syst., vol. 7, pp. 477500, 1996.

[15] F. Wang, J. Wang, C. Zhang, and J. T. Kwok, "Face recognition using spectral features," Pattern Recognition., vol. 40, no. 10, pp. 2786-2797, 2007.

[16] D. G. Lowe, "Distinctive image features from scale invariant keypoints," Int. J. Comput. Vision (IJCV), vol. 60, no. 2, pp. 91-110, 2004. 
[17] L. Zhang, Y. Hu, M. Li, W. Ma, and H. Zhang, "Efficient propagation for face annotation in family albums," in Proc. ACM Int. Conf. Multimedia, 2004, pp. 716-723.

[18] J. Y. Choi, S. Yang, Y. M. Ro, and K. N. Plataniotis, "Face annotation for personal photos using context-assisted face recognition," in Proc.ACM Int. Conf. Multimedia Information Retrieval, 2008, pp. 44-51.

[19] S.-W. Chu, M.-C. Yeh, and K.-T. Cheng, "A real-time, embedded face annotation system," in Proc. ACM Int. Conf. Multimedia, 2008, pp. 989990.

[20] T. F. Cootes, G. J. Edwards, and C. J. Taylor, "Active appearance models," in Proc. ECCV, 1998, vol. 2, pp. 484-498.

[21] Jaewon Sung, Takeo Kande, Daijin Kim, "A Unified Gradient-Based Approach for Combining ASM into AAM" International Journal of Computer Vision 75(2), 297-309, 2007.

[22] A. K. Jain, K. Nandakumar, and A. Ross, "Score normalization in multimodal biometric systems," Pattern Recognition, vol. 38 , no. 12, pp. 2270-2285, 2005.

[23] John Canny, "A computational approach to edge detection", IEEE Transactions on Pattern Analysis and Machine Intellgence, vol. PAMI-8, No.6, November 1986.

[24] Didier Demigny and Tawfik Kamlé, "A Discrete Expression of Canny's Criteria for Step Edge Detector Performances Evaluation" IEEE Transactions on pattern analysis and machine intelligence, vol. 19, no. 11, November 1997. 
[25] Hong-rui Wang, Jian-li Yang, Hai-jun Sun, Dong Chen, Xiu-ling Liu, "An improved Region Growing Method for Medical Image Selection and Evaluation Based on Canny Edge Detection" 978-1-4244-6581$1 / 11 / \$ 26.00$ @2011 IEEE

[26] Herbert Bay, Tinne Tuytelaars, and Luc Van Gool, "SURF: Speeded Up Robust Features" Computer Vision and Image Understanding 110 (2008) 346-359

[27] Shoaib Ehsan, Nadia Kanwal, Adrian F. Clark, Klaus D. McDonald-Maier, "An Algorithm for the Contextual Adaption of SURF Octave Selection With Good Matching Performance: Best Octaves" IEEE Transactions on image processing, vol. 21, no. 1, January 2012.

[28] G. Portera and G. Doran. An anatomical and photographic technique for forensic facial identification. Forensic Science International, 114:97-105, 2000.

[29] S. Z. Li and A. K. Jain (eds.). Handbook of Face Recognition. SpringerVerlag,Secaucus, NJ, 2005.

[30] P. J. Phillips, P. Grother, R. J. Micheals, D. M. Blackburn, E. Tabassi, and M. Bone. Face Recognition Vendor Test 2002: Evaluation Report, Tech. Report NISTIR 6965, NIST, 2003.

[31] G. Yang and T. S. Huang. Human face detection in a scene. In Proc. IEEE Conference on Computer Vision and Pattern Recognition, pages 453-458, 1993. 
[32] M.H. Yang, D. Kriegman, and N. Ahuja. Detecting faces in images: A survey. IEEE Transactions on Pattern Analysis and Machine Intelligence, 24(1):34-58,2002.

[33] R. Chellappa, C. L. Wilson, and S. Sirohey. Human and machine recognition of faces: A survey. Proc. IEEE, 83(5):705-740, 1995.

[34] R.L. Hsu, Mohamed Abdel-Mottaleb, and A. K. Jain. Face detection in color images. IEEE Transactions on Pattern Analysis and Machine Intelligence, 24(5):696-706, 2002.

[35] I. Craw, D. Tock, and A. Bennett. Finding face features. In Proc. European Conference on Computer Vision, pages 92-96, 1992.

[36] H. A. Rowley, S. Baluja, and T. Kanade. Neural network-based face detection. IEEE Transactions on Pattern Analysis and Machine Intelligence, 20(1):23-38,1998.

[37] P. A. Viola and M. J. Jones. Robust real-time face detection. International Journal of Computer Vision, 57(2):137-154, 2004.

[38] K. Levi and Y. Weiss. Learning object detection from a small number of examples: the importance of good features. In Proc. IEEE Conference on Computer Vision and Pattern Recognition, volume 2, pages 53-60, 2002.

[39] B. Heisele, T. Serre, M. Pontil, and T. Poggio. Component-based face detection. In Proc. IEEE Conference on Computer Vision and Pattern Recognition,volume 1, pages 657-662, 2001. 
[40] J. Wu, S. C. Brubaker, M. D. Mullin, and J. M. Rehg. Fast asymmetric learning for cascade face detection. IEEE Transactions on Pattern Analysis and Machine Intelligence, 30(3):369-382, 2008.

[41] Open Computer Vision Library, http://sourceforge.net/projects/opencvlibrary.

[42] M. Kirby and L. Sirovich. Application of the Karhunen-Loeve procedure for the characterization of human faces. IEEE Transactions on Pattern Analysis and Machine Intelligence, 12(1):103-108, 1990.

[43] M. Turk and A. Pentland. Eigenfaces for recognition. Cognitive Neuroscience, 3:72-86, 1991.

[44] W. Zhao, R. Chellappa, P. J. Phillips, and A. Rosenfeld. Face recognition: A literature survey. ACM Computing Surveys (CSUR), 35(4):399-458, 2003.

[45] R. A. Fisher. The statistical utilization of multiple measurements. Annals of Eugenics, 8:376-386, 1938.

[46] L. Wiskott, J.-M. Fellous, N. Kruger, and C. von der Malsburg. Face recognition by elastic bunch graph matching. IEEE Transactions on Pattern Analysis and Machine Intelligence, 19(7):775-779, 1997.

[47] J. P. Lewis. Fast normalized cross-correlation. Vision Interface, pages 120-123, 1995. 
[48] P. J. Phillips, P. Grother, R. J. Micheals, D. M. Blackburn, E. Tabassi, and M. Bone. Face Recognition Vendor Test 2002: Evaluation Report, Tech. Report NISTIR 6965, NIST, 2003.

[49] Neven Vision, FR SDK, http://neven-vision-s-fr-dk.software.informer.com/.

[50] L-1 Identity Solutions, http://www.I1id.com.

[51] J. Stallkamp, H. K. Ekenel, and R. Stiefelhagen. Adaptive background mixture models for real-time tracking. In PProc. IEEE International Conference on Computer Vision, pages 1-8, 2007.

[52] A. Hadid T. Ahonen and M. Pietikainen. Face recognition with local binary patterns. In Proc. European Conference on Computer Vision, pages 469$481,2004$.

[53] W. Zhang, S. Shan, W. Gao, X. Chen, and H. Zhang. Local gabor binary pattern histogram sequence (LGBPHS): A novel non-statistical model for face representation and recognition. In Proc. IEEE International Conference on Computer Vision, pages 786-791, 2005.

[54] L. Wiskott, J.-M. Fellous, N. Kruger, and C. von der Malsburg. Face recognition by elastic bunch graph matching. IEEE Transactions on Pattern Analysis and Machine Intelligence, 19(7):775-779, 1997.

[55] P. S. Penev and J. J. Atick. Local feature analysis: a general statistical theory for object representation. Network: Computation in Neural Systems, 7:477-500, 1996. 
[56] B. S. Manjunath and R. Chellappa. A feature based approach to face recognition. In Proc. IEEE Conference on Computer Vision and Pattern Recognition, pages 373-378, 1992.

[57] S. Arca, P. Campadelli, and R. Lanzarotti. A face recognition system based on local feature analysis. In Proc. Audio- and Video-Based Biometric Person Authentication, pages 182-189, 2003.

[58] J. P. Hespanha P. N. Belhumeur and D. J. Kriegman. Eigenfaces vs. fsherfaces: recognition using class speci- $\mathrm{c}$ linear projection. IEEE Transactions on Pattern Analysis and Machine Intelligence, 19(7):711720, 1997.

[59] N. Vaswani and R. Chellappa. Principal components null space analysis for image and video classification. IEEE Transactions on Image Processing, 15(7):1816-1830, 2006.

[60] M. Grudin. On internal representation in face recognition systems. Pattern Recognition, 33(7):1161-1177, 2000.

[61] K. M. Lam and H. Yan. An analytic-to-holistic approach for face recognition based on a single frontal view. IEEE Transactions on Pattern Analysis and Machine Intelligence, 20(7):673-686, 1998.

[62] B. Heisele, P. Ho, J. Wu, and T. Poggio. Face recognition: componentbased versus global approaches. Computer Vision and Image Understanding, 91(1):6- 21, 2003. 
[63] T.K. Kim, H. Kim, W. Hwang, and J. Kittler. Component-based LDA face description for image retrieval and MPEG-7 standardisation. Image and Vision Computing, 23(7):631-642, 2005.

[64] R. Gottumukkal and V. K. Asari. An improved face recognition technique based on modular pca approach. Pattern Recognition Letters, 25(4):429436, 2004.

[65] X. Geng and Z.H. Zhou. Image region selection and ensemble for face recognition. Journal of Computer Science Technology, 21(1):116-125, 2006.

[66] A. M. Martinez. Recognizing imprecisely localized, partially occluded, and expression variant faces from a single sample per class. IEEE Transactions on Pattern Analysis and Machine Intelligence, 24(6):748763, 2002.

[67] A. Pentland, B. Moghaddam, and T. Starner. View-based and modular eigenspace for face recognition. In Proc. IEEE Conference on Computer Vision and Pattern Recognition, pages 84-91, 1994.

[68] K. Tan and S. Chen. Adaptively weighted sub-pattern PCA for face recognition. Neurocomputing, 64:505-511, 2005.

[69] D. Lin and X. Tang. From macrocosm to microcosm. In Proc. IEEE Conference on Computer Vision and Pattern Recognition, pages 13551362, 2006. 
[70] J. S. Pierrard and T. Vetter. "Skin detail analysis for face recognition." In Proc. IEEE Conference on Computer Vision and Pattern Recognition, pages 1-8, 2007.

[71] P. J. Phillips, W. T. Scruggs, A. J. O'Toole, P. J. Flynn, K.W. Bowyer, C. L. Schott, and M. Sharpe. Face Recognition Vendor Test 2006: FRVT 2006 and ICE 2006 Large-Scale Results Tech. Report NISTIR 7408, NIST, 2007.

[72] FBI Next Generation Identification, http://www.fbi.gov/aboutus/cjis/fingerprintsbiometrics/ngi/ngi2.

[73] U. Park and A. K. Jain. Face matching and retrieval using soft biometrics. IEEE Transactions on Information Forensics and Security, 5(3):406-415, 2010.

[74] M. B. Stegmann, The AAM-API: An open source active appearance model implementation, in Proc. MICCAI, 2003, pp. 951-952.

[75] C. J. Bradley. The Algebra of Geometry: Cartesian, Areal and Projective Coordinates. Bath: High perception, 2007.

[76] Mikolajczyk, K., Schmid, C. "Indexing based on scale invariant interest points." In: ICCV. Volume 1. (2001) $525-531$.

[77] O. Déniz, G. Bueno, J. Salido, F. De la Torre, "Face recognition using Histograms of Oriented Gradients" Pattern Recognition Letters 32 (2011) 1598-1603. 
[78] Krystian Mikolajczyk and Cordelia Schmid, "A performance evaluation of local descriptors" IEEE Transactions on Pattern Analysis and Machine Intelligence, Volume 27 Issue 10, Page 1615-1630, October 2005.

[79] M.A. Turk and A.P. Pentland, "Face Recognition Using Eigenfaces," In Proceedings, IEEE Conference on Computer Vision and Pattern Recognition, pp. 586-591, June 1991.

[80] Samuel Morillas, Valent'ın Gregori and Almanzor Sapena, "Adaptive Marginal Median Filter for Colour Images" Sensors 2011, 11, 3205-3213; doi:10.3390/s110303205.

[81] S. Belongie, J. Malik, and J. Puzicha. "Shape matching and object recognition using shape contexts." IEEE Transactions on Pattern Analysis and Machine Intelligence, 24(4):509-522, 2002.

[82] Jain, V., Mukherjee, A.: The Indian Face Database (2002), http://vis- www.cs.umass.edu/ vidit/IndianFaceDatabase/

[83] Computer Vision laboratory, Faculty of Computer and Information Science, University of Ljubljana, Slovenia, http://www.lrv.fri.unilj.si/facedb.html.

[84] Sim T, Baker S, Bsat M. The CMU pose, "illumination, and expression database." IEEE Transaction on Pattern Analysis and Machine Intelligence. 2003 December;25(12):1615-1618.

[85] T. Sim, S. Baker, and M. Bsat. "The CMU pose, illumination, and expression (PIE) database." In Proceedings of the 5th IEEE International Conference on Face and Gesture Recognition, 2002. 
[86] C. V. van Rijsbergen. "Information Retrieval (2nd ed.)." London: Butterworths, 1979. 


\section{LIST OF PUBLICATIONS}

[1]. Ziaul Haque Choudhury, K.M. Mehata, "Robust Facial Marks Detection Methods Using AAM and SURF" International Journal of Engineering Research and Applications (IJERA), ISSN: 2248-9622, Vol. 2, Issue 6, November- December 2012, pp.708-715.

[2]. Ziaul Haque Choudhury, K.M. Mehata, "Biometrics Security: Facial Marks Detection from the Low Quality Images" International Journal of Computer Applications (0975 - 8887) Volume 66 - No.8, March 2013. 


\section{TECHNICAL BIOGRAPHY}

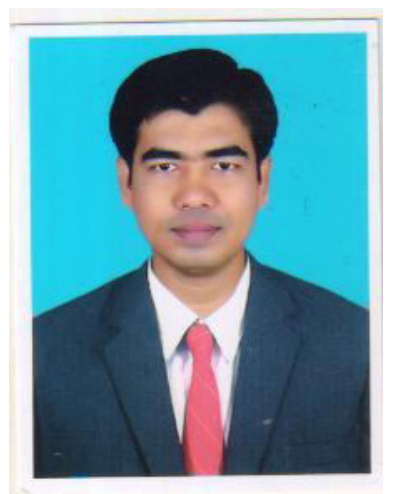

Mr. Ziaul Haque Choudhury (RRN. 1067201) was born on $31^{\text {th }}$ Dec 1980 , in Durgapur, Silchar, Cachar, Assam. He did his schooling in Govt. Boys H.S. School, Silchar. He received B.Sc. degree in Computer Science from Bharathidasan University, Trichy in the year 2007. He did M.Sc.Engineering degree in Information Technology, Department of Computer Science \& Engineering, Annamalai Univerisity, Chidamabaram in the year 2009. He has got one year industrial experience. He was employed (Network Engineer) with Allsec Technologies Ltd., Chennai. He is currently pursuing his M.Tech.(By Research) Degree in Network Security (Biometrics Security) in the Department of Information Technology, B.S. Abdur Rahman University, Chennai. His area of interests includes Network Security, Biometrics Security, Pattern Recognition, Image Processing etc. The e-mail ID is: ziaulms@gmail.com and the contact number is: 9003481822 . 
